\title{
Cluster algebras IV: Coefficients
}

\author{
Sergey Fomin and Andrei Zelevinsky
}

\begin{abstract}
We study the dependence of a cluster algebra on the choice of coefficients. We write general formulas expressing the cluster variables in any cluster algebra in terms of the initial data; these formulas involve a family of polynomials associated with a particular choice of 'principal' coefficients. We show that the exchange graph of a cluster algebra with principal coefficients covers the exchange graph of any cluster algebra with the same exchange matrix. We investigate two families of parameterizations of cluster monomials by lattice points, determined, respectively, by the denominators of their Laurent expansions and by certain multi-gradings in cluster algebras with principal coefficients. The properties of these parameterizations, some proven and some conjectural, suggest links to duality conjectures of Fock and Goncharov. The coefficient dynamics leads to a natural generalization of Zamolodchikov's $Y$-systems. We establish a Laurent phenomenon for such $Y$-systems, previously known in finite type only, and sharpen the periodicity result from an earlier paper. For cluster algebras of finite type, we identify a canonical 'universal' choice of coefficients such that an arbitrary cluster algebra can be obtained from the universal one (of the same type) by an appropriate specialization of coefficients.
\end{abstract}

\section{Contents}

1 Introduction . . . . . . . . . . . . . . . . . . . . . . . 113

2 Preliminaries on cluster algebras . . . . . . . . . . . . . . 115

3 Separation formulas . . . . . . . . . . . . . . . . . . . 119

4 Exchange graphs . . . . . . . . . . . . . . . . . . . 124

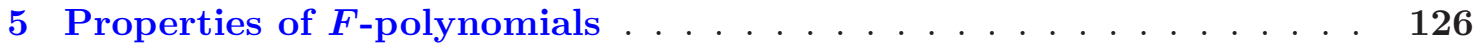

6 Principal $\mathbb{Z}^{n}$-gradings . . . . . . . . . . . . . . . . . . . . . . . 128

7 Cluster monomials and their parameterizations . . . . . . . . . . . 134

8 Bipartite belt and $Y$-systems . . . . . . . . . . . . . . . . . 139

9 Bipartite dynamics on roots . . . . . . . . . . . . . . . 143

10 Denominators and g-vectors on the bipartite belt . . . . . . . . . . 146

11 Finite type . . . . . . . . . . . . . . . . . . . 152

12 Universal coefficients . . . . . . . . . . . . . . . . . 155

13 Index of conjectures . . . . . . . . . . . . . . . . . . . . 163

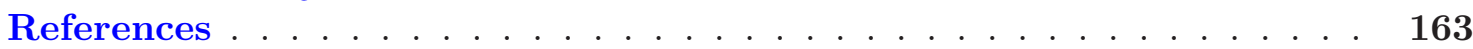

Received 16 March 2006, accepted in final form 2 August 2006.

2000 Mathematics Subject Classification 16S99 (primary), 05E15, 22E46 (secondary).

Keywords: cluster algebra, coefficient semifield, $Y$-system, Laurent polynomial, cluster monomial, root system.

The research of the first author was supported by the NSF grant DMS-0245385. The research of the second author was supported by the NSF grants DMS-0200299 and DMS-0500534, and by a Humboldt Research Award.

This journal is (C) Foundation Compositio Mathematica 2007. 


\section{Cluster algebras IV}

\section{Introduction}

Since their introduction in [FZ02], cluster algebras have found applications in a diverse variety of settings which include (in no particular order) total positivity, Lie theory, quiver representations, Teichmüller theory, Poisson geometry, discrete dynamical systems, tropical geometry, and algebraic combinatorics. See, for example, [CK05a, FG05, FR05, FZ04] and references therein. This paper, the fourth in a series, continues the study of the structural theory of cluster algebras undertaken in its prequels [BFZ05, FZ02, FZ03a]. As in those earlier papers, we try to keep the exposition reasonably self-contained.

A cluster algebra $\mathcal{A}$ of rank $n$ is a subalgebra of an ambient field $\mathcal{F}$ isomorphic to a field of rational functions in $n$ variables. Each cluster algebra comes equipped with a distinguished set of generators called cluster variables; this set is a union of overlapping algebraically independent $n$-subsets of $\mathcal{F}$ called clusters. The clusters are related to each other by birational transformations of the following kind: for every cluster $\mathbf{x}$ and every cluster variable $x \in \mathbf{x}$, there is another cluster $\mathbf{x}^{\prime}=\mathbf{x}-\{x\} \cup\left\{x^{\prime}\right\}$, with the cluster variable $x^{\prime}$ determined by an exchange relation of the form

$$
x x^{\prime}=p^{+} M^{+}+p^{-} M^{-} .
$$

Here $p^{+}$and $p^{-}$belong to a coefficient semifield $\mathbb{P}$, while $M^{+}$and $M^{-}$are two monomials in the elements of $\mathbf{x}-\{x\}$. (See Definition 2.4 for precise details.) Each exchange relation involves two different kinds of data: the exchange matrix $B$ encoding the exponents in $M^{+}$and $M^{-}$, and the two coefficients $p^{+}$and $p^{-}$. In the previous papers of this series, a lot of attention was given to exchange matrices and their dynamics. The current paper brings into focus the dynamics of coefficients. Before describing the results in detail, we would like to offer some justification for the importance and timeliness of this study.

- The coefficient dynamics is of interest in its own right. It can be viewed as a far-reaching generalization of Zamolodchikov's $Y$-systems, introduced and studied in [Zam91] in the context of thermodynamic Bethe Ansatz. Algebraic properties of $Y$-systems were investigated in [FZ03b]; in particular, we established their periodicity, confirming a conjecture by Zamolodchikov (the type $A$ case was settled in [FS95, GT96]). Although our motivation for studying $Y$-systems came from the coefficient dynamics in [FZ02, (5.4)-(5.5)], cluster algebras played no explicit role in [FZ03b]. In the current paper, the study of $Y$-systems in the context of cluster algebras allows us to extend and sharpen the results of [FZ03b].

- Systematically exploring the interplay between two types of dynamics - that of cluster variables and that of coefficients - leads to a better understanding of both phenomena. The constructions we use to express this interplay are close in spirit (albeit different in technical details) to the notion of cluster ensemble introduced and studied by Fock and Goncharov [FG03] as a tool in higher Teichmüller theory. Our coefficient-based approach uncovers an unexpected 'common source' of the two types of dynamics, expressing both the cluster variables and the coefficients in terms of a new family of $F$-polynomials, which generalize the Fibonacci polynomials of [FZ03b]. This approach also yields a new constructive way to express the (conjectural) 'Langlands duality' between the two kinds of dynamics, suggested in [FG03].

- Last, but not least, all examples of cluster algebras coming from geometry of semisimple groups (see [BFZ05, FZ03a, FZ04]) have non-trivial coefficients. (By contrast, most of the recent developments in cluster algebra theory obtained in the context of cluster categories and cluster tilted algebras deal exclusively with the case of trivial coefficients.) There are many examples of cluster algebras of geometric origin which have the same combinatorics of exchange matrices but totally different systems of coefficients. This motivates the study of the dependence of a cluster algebra structure on the choice of coefficients. One surprising conclusion that we reach in this study is the existence of 'universal formulas' for cluster variables; these formulas reduce the case of arbitrary coefficients to a very particular special case of principal coefficients. 


\section{S. Fomin And A. Zelevinsky}

The paper is organized as follows. Requisite background on cluster algebras is presented in $\S 2$. In keeping with the tradition established in [BFZ05, FZ02, FZ03a], we rephrase the definitions to better suit our current purposes. In particular, we reconcile the setups in [FZ02] and [FZ03a] by emphasizing the difference between the labeled seeds attached to the vertices of an $n$-regular tree (see Definitions 2.3 and 2.8), and the (unlabeled) seeds attached to the vertices of an exchange graph (see Definitions 4.1 and 4.2). (Informally, a seed is a cluster $\mathbf{x}$ together with the corresponding exchange matrix $B$ and the collection of coefficients $p^{ \pm}$appearing in all exchanges from $\mathbf{x}$.)

In $\S 3$, we introduce some of the main new concepts that play a central role in the paper, most notably, the cluster algebras with principal coefficients (Definition 3.1) and, based on the latter, the $F$-polynomials (Definition 3.3). Our first main result, Theorem 3.7 (sharpened in Corollary 6.3), expresses any cluster variable in any cluster algebra in terms of the (arbitrary) initial seed using the appropriate $F$-polynomials. Thus, one can think of principal coefficients as a crucial special case providing control over cluster algebras with arbitrary coefficients.

The universal formulas of Theorem 3.7/Corollary 6.3 have an a priori unexpected 'separation of additions' property: each cluster variable is written as a ratio of two polynomial expressions in the initial data, one of the them (the numerator) employing only the 'ordinary addition' in $\mathcal{F}$, and another one (the denominator) involving only the 'auxiliary addition' in the coefficient semifield $\mathbb{P}$.

These formulas lead to the following structural result obtained in $\S 4$ (Theorem 4.6): the exchange graph of an algebra with principal coefficients serves as a cover for the exchange graph of any cluster algebra with the same exchange matrix. This is a step towards the much stronger Conjecture 4.3: the exchange graph of a cluster algebra depends only on the exchange matrix at any seed, but not on the choice of coefficients.

Properties of $F$-polynomials are discussed in $\S 5$. Although the definition of $F$-polynomials is simple and elementary, proving some of their properties resisted our efforts. One example is Conjecture 5.4, an innocent-looking assertion that every $F$-polynomial has constant term 1.

In $\S 6$, we show that a cluster algebra with principal coefficients possesses a $\mathbb{Z}^{n}$-grading with respect to which all cluster variables are homogeneous elements. We then discuss general properties (partly proven and partly conjectural) of the (multi-)degrees of cluster variables, which we refer to as g-vectors. In particular, we show that these vectors can be expressed in terms of (tropical evaluations of) $F$-polynomials.

Section 7 applies the tools developed in earlier sections to the study of cluster monomials, that is, monomials in cluster variables all of which belong to the same cluster. The significance of cluster monomials stems from the expectation that they belong to the suitably defined 'canonical basis' of a cluster algebra. There is still a long way to go towards this result: even the linear independence of cluster monomials remains open (see Conjecture 7.2, reproducing [FZ04, Conjecture 4.16]). As a possible tool in proving this conjecture, we discuss two families of combinatorial parameterizations of cluster monomials by integer vectors in $\mathbb{Z}^{n}$; they are somewhat reminiscent of two families of parameterizations of canonical bases (Lusztig's and string parameterizations) in the theory of quantum groups. One of these parameterizations of cluster monomials (by denominator vectors) has already appeared in [FZ03a, FZ04]; another (by g-vectors) is new. Note that in $\S 6$, g-vectors are defined in restricted generality, for the case of principal coefficients only; in $\S 7$, we find a way to extend this definition to a much more general class of coefficients.

In the rest of the paper $(\S \S 8-12)$, we restrict our attention to some special classes of cluster algebras. Sections 8-10 deal with the bipartite cluster algebras, a class that includes all cluster algebras of finite type. A bipartite cluster algebra has a seed $\Sigma$ such that, in each of the $n$ exchange relations (1.1) emanating from $\Sigma$, one of the monomials $M^{+}$and $M^{-}$is equal to 1 . Such a seed $\Sigma$ is also called bipartite, and is naturally included into a sequence of bipartite seeds 


\section{Cluster algebras IV}

called the bipartite belt. In $\S 8$, we show that the restriction of the coefficient dynamics to the bipartite belt yields a natural generalization of Zamolodchikov's $Y$-systems to the case of arbitrary symmetrizable generalized Cartan matrices. Two main results of this section sharpen their counterparts obtained in [FZ03b]: Theorem 8.5 (the 'Laurent phenomenon') asserts that the solutions of a generalized $Y$-system can be expressed as Laurent polynomials in the initial data, while Theorem 8.8 improves upon the periodicity theorem of [FZ03b] by showing the necessity of the finite type assumption.

The proof of Theorem 8.8 given in $\S 10$ relies on the calculation of denominator vectors and g-vectors for the cluster variables on the bipartite belt. We also need some properties of the root system associated with a symmetrizable generalized Cartan matrix. These properties are discussed in $\S 9$, which can be read independently of the rest of the paper. We believe these results to be of independent interest. In particular, Theorem 9.4 and Corollary 9.6 strengthen the following result obtained in [BLM89]: in the Weyl group associated to an indecomposable symmetrizable Cartan matrix of infinite type, the 'bipartite' Coxeter element has infinite order.

The last two sections ( $\S 11$ and 12) deal with the cluster algebras of finite type, i.e. those with finitely many seeds. The classification of these algebras obtained in [FZ03a] is parallel to the CartanKilling classification of semisimple Lie algebras and finite root systems. In $\S 11$, we demonstrate that several conjectures made in the preceding sections hold for the algebras of finite type. In particular, we prove linear independence of cluster monomials (Conjecture 7.2) for the cluster algebras of finite type; see Theorem 11.2. Other conjectures are proved by combining our results on bipartite cluster algebras with the structural results in [FZ03a], such as the following property (implicit in [FZ03a]): in a cluster algebra of finite type, every cluster variable appears in a seed lying on the bipartite belt.

In Theorem 11.6, we show that in finite type, the $F$-polynomials coincide, up to a simple twist, with the Fibonacci polynomials of [FZ03b].

The concluding section $(\S 12)$ presents an explicit construction of the 'universal' system of coefficients for cluster algebras of each finite type. This universality is understood in the following sense. As a consequence of results in [FZ03a], for any two cluster algebras $\mathcal{A}$ and $\overline{\mathcal{A}}$ of the same (finite) Cartan-Killing type, there is a natural bijection between the sets of cluster variables for $\mathcal{A}$ and $\overline{\mathcal{A}}$. So the only feature that distinguishes these algebras from each other is a choice of coefficients (in turn predicated on the choice of the ground ring, the integral group ring $\mathbb{Z} \mathbb{P}$ of the multiplicative group of the coefficient semifield $\mathbb{P}$ ). The main result of $\S 12$ (Theorem 12.4) asserts that, among all cluster algebras of a given Cartan-Killing type, there is a universal cluster algebra $\mathcal{A}^{\text {univ }}$ such that every other algebra of this type is obtained from $\mathcal{A}^{\text {univ }}$ by a (unique) 'change of base' resulting from a homomorphism of multiplicative groups of coefficient semifields. The existence of $\mathcal{A}^{\text {univ }}$ is by no means obvious, and it is not clear how far this result can be extended beyond finite type.

The reader will notice a large number of conjectures put forth in $\S \S 4-7$. We conclude the paper by an index of partial results that we obtained towards these conjectures. We note that a promising tool for attacking some of these conjectures is provided by a geometric interpretation (found in [CC04] and generalized in [CK05b]) of Laurent expansions of cluster variables in terms of Grassmannians of quiver representations.

\section{Preliminaries on cluster algebras}

To state our results, we recall the basic setup introduced in [BFZ05, FZ02, FZ03a]. In keeping with the tradition established in the previous papers in the series, we rephrase the definitions to better suit the current purposes. 


\section{S. Fomin And A. Zelevinsky}

The definition of a cluster algebra $\mathcal{A}$ starts with introducing its ground ring. Let $(\mathbb{P}, \oplus, \cdot)$ be a semifield, i.e. an abelian multiplicative group endowed with a binary operation of (auxiliary) addition $\oplus$ which is commutative, associative, and distributive with respect to the multiplication in $\mathbb{P}$. The multiplicative group of $\mathbb{P}$ is torsion-free $[\mathrm{FZ02}, \S 5]$, hence its group ring $\mathbb{Z} \mathbb{P}$ - which will be used as a ground ring for $\mathcal{A}$ - is a domain.

The following two examples of semifields will be of particular importance to us.

Definition 2.1 (Universal semifield). Let $\mathbb{Q}_{\mathrm{sf}}\left(u_{1}, \ldots, u_{\ell}\right)$ denote the set of all rational functions in $\ell$ independent variables $u_{1}, \ldots, u_{\ell}$ which can be written as subtraction-free rational expressions in $u_{1}, \ldots, u_{\ell}$. For example, $u^{2}-u+1=\left(u^{3}+1\right) /(u+1) \in \mathbb{Q}_{\mathrm{sf}}(u)$. The set $\mathbb{Q}_{\mathrm{sf}}\left(u_{1}, \ldots, u_{\ell}\right)$ is a semifield with respect to the usual operations of multiplication and addition. This example is universal: any subtraction-free identity that holds in $\mathbb{Q}_{\mathrm{sf}}\left(u_{1}, \ldots, u_{\ell}\right)$ remains valid for any elements $u_{1}, \ldots, u_{\ell}$ in an arbitrary semifield (see [BFZ96, Lemma 2.1.6]).

Definition 2.2 (Tropical semifield). Let $J$ be a finite set of labels, and let $\operatorname{Trop}\left(u_{j}: j \in J\right)$ be an abelian group (written multiplicatively) freely generated by the elements $u_{j}(j \in J)$. We define the addition $\oplus$ in $\operatorname{Trop}\left(u_{j}: j \in J\right)$ by

$$
\prod_{j} u_{j}^{a_{j}} \oplus \prod_{j} u_{j}^{b_{j}}=\prod_{j} u_{j}^{\min \left(a_{j}, b_{j}\right)},
$$

and call $\left(\operatorname{Trop}\left(u_{j}: j \in J\right), \oplus, \cdot\right)$ a tropical semifield. To illustrate, $u_{2} \oplus u_{1}^{2} u_{2}^{-1}=u_{2}^{-1}$ in $\operatorname{Trop}\left(u_{1}, u_{2}\right)$. If $J$ is empty, we obtain the trivial semifield consisting of a single element 1.

The group ring of $\operatorname{Trop}\left(u_{j}: j \in J\right)$ is the ring of Laurent polynomials in the variables $u_{j}$.

The terminology in Definition 2.2 is consistent with [FZ02]. It is different from (although closely related to) the terminology in [BFZ96, § 2.1], nowadays commonly used in tropical geometry.

As an ambient field for a cluster algebra $\mathcal{A}$, we take a field $\mathcal{F}$ isomorphic to the field of rational functions in $n$ independent variables (here $n$ is the rank of $\mathcal{A}$ ), with coefficients in $\mathbb{Q P}$. Note that the definition of $\mathcal{F}$ ignores the auxiliary addition in $\mathbb{P}$.

Definition 2.3 (Labeled seeds). A (skew-symmetrizable) labeled $Y$-seed in $\mathbb{P}$ is a pair $(\mathbf{y}, B)$, where:

- $\mathbf{y}=\left(y_{1}, \ldots, y_{n}\right)$ is an $n$-tuple of elements of $\mathbb{P}$; and

- $B=\left(b_{i j}\right)$ is an $n \times n$ integer matrix which is skew-symmetrizable.

That is, $d_{i} b_{i j}=-d_{j} b_{j i}$ for some positive integers $d_{1}, \ldots, d_{n}$. A labeled seed in $\mathcal{F}$ is a triple $(\mathbf{x}, \mathbf{y}, B)$, where:

- $(\mathbf{y}, B)$ is a labeled $Y$-seed; and

- $\mathbf{x}=\left(x_{1}, \ldots, x_{n}\right)$ is an $n$-tuple of elements of $\mathcal{F}$ forming a free generating set.

That is, $x_{1}, \ldots, x_{n}$ are algebraically independent over $\mathbb{Q P}$, and $\mathcal{F}=\mathbb{Q P}\left(x_{1}, \ldots, x_{n}\right)$. We refer to $\mathbf{x}$ as the (labeled) cluster of a labeled seed $(\mathbf{x}, \mathbf{y}, B)$, to the tuple $\mathbf{y}$ as the coefficient tuple, and to the matrix $B$ as the exchange matrix.

The (unlabeled) seeds, as defined in [FZ03a] and in Definition 4.1 below, are obtained by identifying labeled seeds that differ from each other by simultaneous permutations of the components in $\mathbf{x}$ and $\mathbf{y}$, and of the rows and columns of $B$. In this paper, we mostly deal with labeled seeds; meanwhile, we sometimes refer to labeled seeds simply as seeds, when there is no risk of confusion. 


\section{Cluster algebras IV}

Throughout the paper, we use the notation

$$
\begin{gathered}
{[x]_{+}=\max (x, 0)} \\
\operatorname{sgn}(x)=\left\{\begin{array}{rc}
-1 & \text { if } x<0 \\
0 & \text { if } x=0 \\
1 & \text { if } x>0
\end{array}\right. \\
{[1, n]=\{1, \ldots, n\} .}
\end{gathered}
$$

Definition 2.4 (Seed mutations). Let $(\mathbf{x}, \mathbf{y}, B)$ be a labeled seed in $\mathcal{F}$, as in Definition 2.3 above, and let $k \in[1, n]$. The seed mutation $\mu_{k}$ in direction $k$ transforms $(\mathbf{x}, \mathbf{y}, B)$ into the labeled seed $\mu_{k}(\mathbf{x}, \mathbf{y}, B)=\left(\mathbf{x}^{\prime}, \mathbf{y}^{\prime}, B^{\prime}\right)$ defined as follows.

- The entries of $B^{\prime}=\left(b_{i j}^{\prime}\right)$ are given by

$$
b_{i j}^{\prime}= \begin{cases}-b_{i j} & \text { if } i=k \text { or } j=k ; \\ b_{i j}+\operatorname{sgn}\left(b_{i k}\right)\left[b_{i k} b_{k j}\right]_{+} & \text {otherwise. }\end{cases}
$$

- The coefficient tuple $\mathbf{y}^{\prime}=\left(y_{1}^{\prime}, \ldots, y_{n}^{\prime}\right)$ is given by

$$
y_{j}^{\prime}= \begin{cases}y_{k}^{-1} & \text { if } j=k ; \\ y_{j} y_{k}^{\left[b_{k j}\right]_{+}}\left(y_{k} \oplus 1\right)^{-b_{k j}} & \text { if } j \neq k .\end{cases}
$$

- The cluster $\mathbf{x}^{\prime}=\left(x_{1}^{\prime}, \ldots, x_{n}^{\prime}\right)$ is given by $x_{j}^{\prime}=x_{j}$ for $j \neq k$, whereas $x_{k}^{\prime} \in \mathcal{F}$ is determined by the exchange relation

$$
x_{k}^{\prime}=\frac{y_{k} \prod x_{i}^{\left[b_{i k}\right]_{+}}+\prod x_{i}^{\left[-b_{i k}\right]_{+}}}{\left(y_{k} \oplus 1\right) x_{k}} .
$$

We also say that the $Y$-seed mutation in direction $k$ transforms $(\mathbf{y}, B)$ into the labeled $Y$-seed $\mu_{k}(\mathbf{y}, B)=\left(\mathbf{y}^{\prime}, B^{\prime}\right)$ given by $(2.2)$ and $(2.3)$.

It is easy to see that $B^{\prime}$ is skew-symmetrizable (with the same choice of $d_{1}, \ldots, d_{n}$ ), implying that $\left(\mathbf{x}^{\prime}, \mathbf{y}^{\prime}, B^{\prime}\right)$ is indeed a labeled seed. Furthermore, the seed mutation $\mu_{k}$ is involutive, that is, it transforms $\left(\mathbf{x}^{\prime}, \mathbf{y}^{\prime}, B^{\prime}\right)$ into the original labeled seed $(\mathbf{x}, \mathbf{y}, B)$.

A few comments are in order.

Remark 2.5. The transformation $\mu_{k}: B \mapsto B^{\prime}$ defined by (2.2) is easily checked to coincide with the matrix mutation defined in [FZ02, (4.3)]. Another equivalent way to define it is

$$
b_{i j}^{\prime}= \begin{cases}-b_{i j} & \text { if } i=k \text { or } j=k ; \\ b_{i j}+\left[-b_{i k}\right]_{+} b_{k j}+b_{i k}\left[b_{k j}\right]_{+} & \text {otherwise. }\end{cases}
$$

Remark 2.6. The transformation $\mathbf{y} \mapsto \mathbf{y}^{\prime}$ given by (2.3) first appeared in [FZ02, (5.5)], and then in a different context in [GSV03, Lemma 1.3] and [FG03, (6)] (cf. Proposition 3.9 below).

Remark 2.7. Originally [FZ02, Definition 5.3; FZ03a, $§ 1.2]$, we defined the coefficient tuple as a $2 n$-tuple $\mathbf{p}=\left(p_{1}^{ \pm}, \ldots, p_{n}^{ \pm}\right)$of elements of $\mathbb{P}$ satisfying the normalization condition $p_{j}^{+} \oplus p_{j}^{-}=1$ for all $j$. The equivalence of that setup and the current one was established in [FZ02, (5.2) and (5.3)]: by setting

$$
y_{j}=\frac{p_{j}^{+}}{p_{j}^{-}}
$$

the coefficients $p_{j}^{ \pm}$are recovered via

$$
p_{j}^{+}=\frac{y_{j}}{y_{j} \oplus 1}, \quad p_{j}^{-}=\frac{1}{y_{j} \oplus 1},
$$




\section{S. Fomin And A. Zelevinsky}

and the exchange relation (2.4) takes the usual form (cf. [FZ03a, Definition 1.1])

$$
x_{k}^{\prime}=x_{k}^{-1}\left(p_{k}^{+} \prod_{i=1}^{n} x_{i}^{\left[b_{i k}\right]_{+}}+p_{k}^{-} \prod_{i=1}^{n} x_{i}^{\left[-b_{i k}\right]_{+}}\right) .
$$

Definition 2.8 (Regular $n$-ary tree). As in [FZ02], we consider the $n$-regular tree $\mathbb{T}_{n}$ whose edges are labeled by the numbers $1, \ldots, n$, so that the $n$ edges emanating from each vertex receive different labels. We write $t \stackrel{k}{-} t^{\prime}$ to indicate that vertices $t, t^{\prime} \in \mathbb{T}_{n}$ are joined by an edge labeled by $k$.

Definition 2.9 (Patterns). A cluster pattern (respectively, $Y$-pattern) is an assignment of a labeled seed $\Sigma_{t}=\left(\mathbf{x}_{t}, \mathbf{y}_{t}, B_{t}\right)$ (respectively, a labeled $Y$-seed $\left.\left(\mathbf{y}_{t}, B_{t}\right)\right)$ to every vertex $t \in \mathbb{T}_{n}$, such that the seeds assigned to the endpoints of any edge $t \stackrel{k}{-} t^{\prime}$ are obtained from each other by the seed mutation in direction $k$. The elements of $\Sigma_{t}$ are written as follows:

$$
\mathbf{x}_{t}=\left(x_{1 ; t}, \ldots, x_{n ; t}\right), \quad \mathbf{y}_{t}=\left(y_{1 ; t}, \ldots, y_{n ; t}\right), \quad B_{t}=\left(b_{i j}^{t}\right) .
$$

Clearly, a cluster pattern (respectively, $Y$-pattern) is uniquely determined by each of its seeds (respectively, $Y$-seeds), which can be chosen arbitrarily.

As will be explained in $\S 8$, the notion of a $Y$-pattern is a far-reaching generalization of Zamolodchikov's $Y$-systems [FZ03b, Zam91].

Example 2.10 (Type $A_{2}$; cf. [FZ02, Section 6]). Let $n=2$. Then the tree $\mathbb{T}_{2}$ is an infinite chain. We denote its vertices by $\ldots, t_{-1}, t_{0}, t_{1}, t_{2}, \ldots$, and label its edges as follows:

$$
\cdots \stackrel{2}{2} t_{-1} \stackrel{1}{-} t_{0} \stackrel{2}{-} t_{1} \stackrel{1}{-} t_{2} \stackrel{2}{-} t_{3} \stackrel{1}{-} \cdots \text {. }
$$

We denote the corresponding seeds by $\Sigma_{m}=\Sigma_{t_{m}}=\left(\mathbf{x}_{m}, \mathbf{y}_{m}, B_{m}\right)$, for $m \in \mathbb{Z}$. Let the initial seed $\Sigma_{0}$ be

$$
\mathbf{x}_{0}=\left(x_{1}, x_{2}\right), \quad \mathbf{y}_{0}=\left(y_{1}, y_{2}\right), \quad B_{0}=\left[\begin{array}{rr}
0 & 1 \\
-1 & 0
\end{array}\right]
$$

We then recursively compute the seeds $\Sigma_{1}, \ldots, \Sigma_{5}$ as shown in Table 1 . Note that the labeled seed $\Sigma_{5}$ is obtained from $\Sigma_{0}$ by interchanging the indices 1 and 2 ; the sequence then continues by obvious periodicity (so that $\Sigma_{10}$ becomes identical to $\Sigma_{0}$, etc.).

Now everything is in place for defining cluster algebras.

Definition 2.11 (Cluster algebra). Given a cluster pattern, we denote by

$$
\mathcal{X}=\bigcup_{t \in \mathbb{T}_{n}} \mathbf{x}_{t}=\left\{x_{i, t}: t \in \mathbb{T}_{n}, 1 \leqslant i \leqslant n\right\}
$$

the union of clusters of all of the seeds in the pattern. We refer to the elements $x_{i, t} \in \mathcal{X}$ as cluster variables. The cluster algebra $\mathcal{A}$ associated with a given cluster pattern is the $\mathbb{Z} \mathbb{P}$-subalgebra of the ambient field $\mathcal{F}$ generated by all cluster variables: $\mathcal{A}=\mathbb{Z} \mathbb{P}[\mathcal{X}]$. We denote $\mathcal{A}=\mathcal{A}(\mathbf{x}, \mathbf{y}, B)$, where $(\mathbf{x}, \mathbf{y}, B)=\left(\mathbf{x}_{t}, \mathbf{y}_{t}, B_{t}\right)$ is any labeled seed in the underlying cluster pattern.

Definition 2.12 (Geometric type). A cluster algebra (or a $Y$-pattern, or a cluster pattern) is of geometric type if the coefficient semifield $\mathbb{P}$ is a tropical semifield of Definition 2.2.

For patterns of geometric type, it is convenient to denote the generators of $\mathbb{P}$ by $x_{n+1}, \ldots, x_{m}$ (for some integer $m \geqslant n$ ), so that $\mathbb{P}=\operatorname{Trop}\left(x_{n+1}, \ldots, x_{m}\right)$. As the coefficients $y_{1 ; t}, \ldots, y_{n ; t}$ at each seed $\Sigma_{t}=\left(\mathbf{x}_{t}, \mathbf{y}_{t}, B_{t}\right)$ are Laurent monomials in $x_{n+1}, \ldots, x_{m}$, we define the integers $b_{i j}^{t}$, for $j \in[1, n]$ and $n<i \leqslant m$, by

$$
y_{j ; t}=\prod_{i=n+1}^{m} x_{i}^{b_{i j}^{t}}
$$




\section{Cluster algebras IV}

TABLE 1 . Seeds in type $A_{2}$.

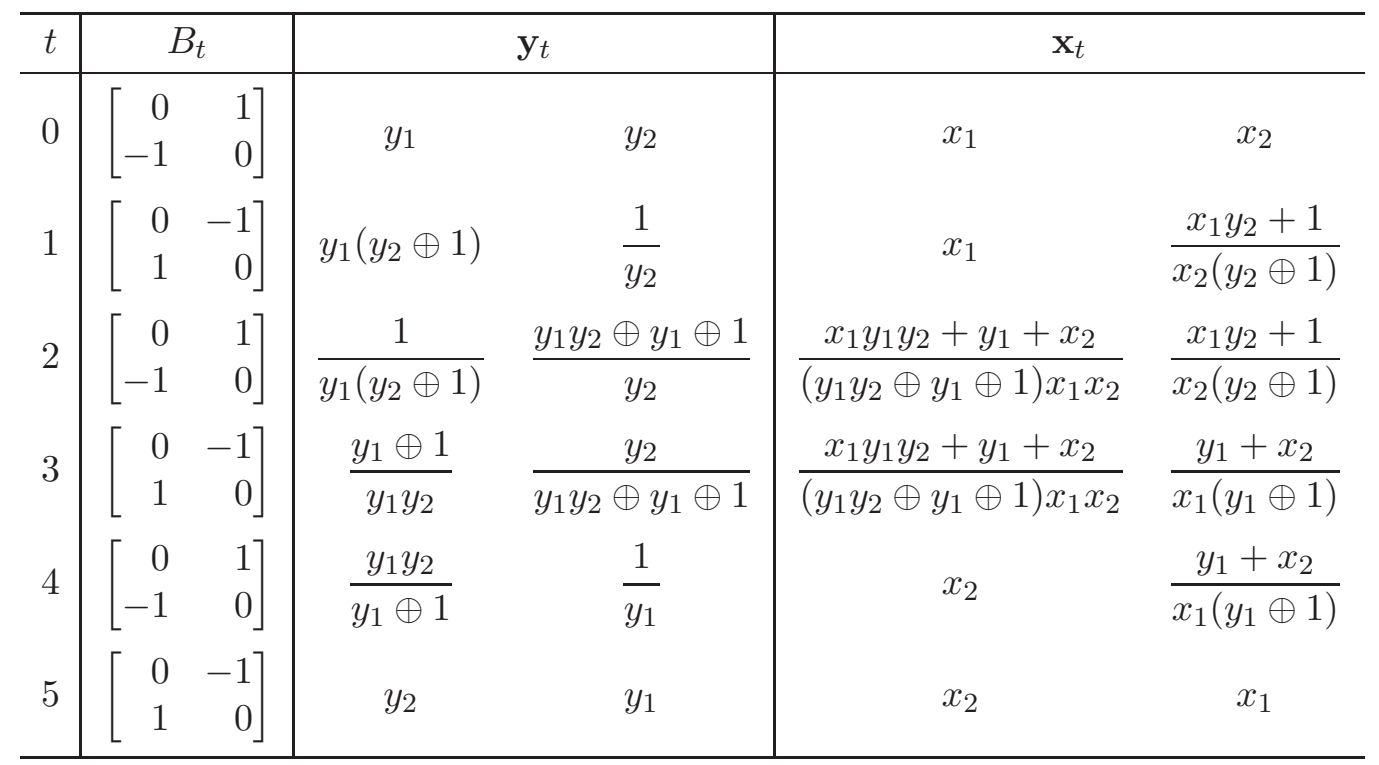

That is, we include the exchange matrix $B_{t}$ as a submatrix into a larger $m \times n$ matrix

$$
\tilde{B}_{t}=\left(b_{i j}^{t}\right) \quad(1 \leqslant i \leqslant m, 1 \leqslant j \leqslant n)
$$

whose matrix elements $b_{i j}^{t}$ with $i>n$ are used to encode the coefficients $y_{j}=y_{j ; t}$. It then follows from Definition 2.2 that the formulas (2.7) for a seed $\Sigma_{t}$ become

$$
p_{j}^{+}=\prod_{i=n+1}^{m} x_{i}^{\left[b_{i j}^{t}\right]_{+}}, \quad p_{j}^{-}=\prod_{i=n+1}^{m} x_{i}^{\left[-b_{i j}^{t}\right]_{+}},
$$

and the exchange relations (2.8) become

$$
x_{k}^{\prime}=x_{k}^{-1}\left(\prod_{i=1}^{m} x_{i}^{\left[b_{i k}^{t}\right]_{+}}+\prod_{i=1}^{m} x_{i}^{\left[-b_{i k}^{t}\right]_{+}}\right),
$$

where we denote $x_{i}=x_{i ; t}$ for $i \in[1, n]$. Furthermore, the $Y$-seed mutation formulas (2.3) take the form (2.2), with $i>n$ (cf. [FZ02, Proposition 5.8]). Thus, extending the notion of matrix mutation to rectangular matrices, we have $\tilde{B}_{t^{\prime}}=\mu_{k}\left(\tilde{B}_{t}\right)$ whenever $t \stackrel{k}{t} t^{\prime}$. Summing up, the entire $Y$-pattern (hence, up to isomorphism, the corresponding cluster algebra of geometric type) is uniquely determined by a single rectangular matrix $\tilde{B}_{t}$.

\section{Separation formulas}

We now introduce a special class of coefficient patterns that will play a central role in this paper.

Definition 3.1 (Principal coefficients). We say that a $Y$-pattern $t \mapsto\left(\mathbf{y}_{t}, B_{t}\right)$ on $\mathbb{T}_{n}$ (or a cluster pattern $t \mapsto\left(\mathbf{x}_{t}, \mathbf{y}_{t}, B_{t}\right)$, or the corresponding cluster algebra $\left.\mathcal{A}\right)$ has principal coefficients at a vertex $t_{0}$ if $\mathbb{P}=\operatorname{Trop}\left(y_{1}, \ldots, y_{n}\right)$ and $\mathbf{y}_{t_{0}}=\left(y_{1}, \ldots, y_{n}\right)$. In this case, we denote $\mathcal{A}=\mathcal{A}_{\bullet}\left(B_{t_{0}}\right)$.

Remark 3.2. Definition 3.1 can be rephrased as follows: a cluster algebra $\mathcal{A}$ has principal coefficients at a vertex $t_{0}$ if $\mathcal{A}$ is of geometric type, and is associated with the matrix $\tilde{B}_{t_{0}}$ of order $2 n \times n$ whose principal (i.e. top $n \times n$ ) part is $B_{t_{0}}$, and whose complementary (i.e. bottom) part is the $n \times n$ identity matrix (cf. [FZ02, Corollary 5.9]). 


\section{S. Fomin And A. Zelevinsky}

In Theorem 3.7 below we give a formula that expresses a cluster variable in an arbitrary cluster algebra with a given initial exchange matrix $B^{0}=B_{t_{0}}$ in terms of similar formulas for the corresponding cluster algebra with principal coefficients. In order to write these formulas, we need to introduce some notation.

Definition 3.3 (The functions $X_{\ell, t}$ and $\left.F_{\ell, t}\right)$. Let $\mathcal{A}=\mathcal{A}_{\bullet}\left(B^{0}\right)$ be the cluster algebra with principal coefficients at a vertex $t_{0}$, defined by the initial seed $\Sigma_{t_{0}}=\left(\mathbf{x}_{t_{0}}, \mathbf{y}_{t_{0}}, B_{t_{0}}\right)$ with

$$
\mathbf{x}_{t_{0}}=\left(x_{1}, \ldots, x_{n}\right), \quad \mathbf{y}_{t_{0}}=\left(y_{1}, \ldots, y_{n}\right), \quad B_{t_{0}}=B^{0}=\left(b_{i j}^{0}\right) .
$$

Thus, $\mathbb{P}=\operatorname{Trop}\left(y_{1}, \ldots, y_{n}\right)$, and all coefficients in all exchange relations (2.4) are monomials in $y_{1}, \ldots, y_{n}$. By iterating these exchange relations, we can express every cluster variable $x_{\ell ; t}$ as a (unique) rational function in $x_{1}, \ldots, x_{n}, y_{1}, \ldots, y_{n}$ given by a subtraction-free rational expression; we denote this rational function by

$$
X_{\ell ; t}=X_{\ell ; t}^{B^{0} ; t_{0}} \in \mathbb{Q}_{\mathrm{sf}}\left(x_{1}, \ldots, x_{n} ; y_{1}, \ldots, y_{n}\right) .
$$

Let $F_{\ell ; t}=F_{\ell ; t}^{B^{0} ; t_{0}} \in \mathbb{Q}_{\mathrm{sf}}\left(y_{1}, \ldots, y_{n}\right)$ denote the rational function obtained from $X_{\ell ; t}$ by specializing all of the $x_{j}$ to 1 :

$$
F_{\ell ; t}\left(y_{1}, \ldots, y_{n}\right)=X_{\ell ; t}\left(1, \ldots, 1 ; y_{1}, \ldots, y_{n}\right)
$$

For instance, we have $X_{\ell ; t_{0}}=x_{\ell}$ and $F_{\ell ; t_{0}}=1$ for all $\ell$; and if $t_{1} \stackrel{k}{t} t_{0}$, then

$$
X_{k ; t_{1}}^{B^{0} ; t_{0}}=\frac{y_{k} \prod x_{i}^{\left[b_{i k}^{0}\right]_{+}}+\prod x_{i}^{\left[-b_{i k}^{0}\right]_{+}}}{x_{k}}, \quad F_{k ; t_{1}}^{B^{0} ; t_{0}}=y_{k}+1 .
$$

Example 3.4 (Principal coefficients, type $A_{2}$ ). Let $n=2$ and $B^{0}=\left[\begin{array}{cc}0 & 1 \\ -1 & 0\end{array}\right]$. That is, we consider the special case of Example 2.10 in which the coefficient semifield is $\mathbb{P}=\operatorname{Trop}\left(y_{1}, y_{2}\right)$. In this case, the formulas in Table 1 simplify considerably. The results are shown in the first four columns of Table 2 , where we use the notation $\mathbf{x}_{t}=\left(X_{1 ; t}, X_{2 ; t}\right)$, in agreement with Definition 3.3. Setting $x_{1}=x_{2}=1$ yields the polynomials $F_{i ; t}$, shown in the fifth column of the table.

As we now demonstrate, each rational function $X_{\ell ; t}$ is in fact a Laurent polynomial with integer coefficients, while each $F_{\ell ; t}$ is actually a polynomial in $y_{1}, \ldots, y_{n}$.

Theorem 3.5 (Laurent phenomenon [FZ02, Theorem 3.1]). The cluster algebra $\mathcal{A}$ associated with a seed $(\mathbf{x}, \mathbf{y}, B)$ is contained in the Laurent polynomial ring $\mathbb{Z} \mathbb{P}\left[\mathbf{x}^{ \pm 1}\right]$, i.e. every element of $\mathcal{A}$ is a Laurent polynomial over $\mathbb{Z} \mathbb{P}$ in the cluster variables from $\mathbf{x}$.

In view of [FZ03a, Proposition 11.2], for a cluster algebra with principal coefficients, Theorem 3.5 can be sharpened as follows.

Proposition 3.6. Let $\mathcal{A}=\mathcal{A}_{\bullet}\left(B^{0}\right)$ be the cluster algebra with principal coefficients at a vertex $t_{0}$ with the initial seed given by (3.1). Then $\mathcal{A} \subset \mathbb{Z}\left[x_{1}^{ \pm 1}, \ldots, x_{n}^{ \pm 1} ; y_{1}, \ldots, y_{n}\right]$. That is, every element of $\mathcal{A}$ is a Laurent polynomial in $x_{1}, \ldots, x_{n}$ whose coefficients are integer polynomials in $y_{1}, \ldots, y_{n}$. Thus,

$$
X_{\ell ; t} \in \mathbb{Z}\left[x_{1}^{ \pm 1}, \ldots, x_{n}^{ \pm 1} ; y_{1}, \ldots, y_{n}\right], \quad F_{\ell ; t} \in \mathbb{Z}\left[y_{1}, \ldots, y_{n}\right] .
$$

The polynomials in (3.4) are conjectured to have positive coefficients. We note that not until the positivity of coefficients of the (Laurent) polynomial expansion for some $X_{j ; t}$ (or $F_{j ; t}$ ) has been established, can such an expansion be used for evaluation in an arbitrary semifield. On the other hand, one can always use an appropriate subtraction-free expression. 


\section{Cluster algebras IV}

TABLE 2. Type $A_{2}$, principal coefficients.

\begin{tabular}{|c|c|c|c|c|c|c|c|}
\hline$t$ & $\tilde{B}_{t}$ & $\mathbf{y}_{t}$ & & $X_{1 ; t}$ & $X_{2 ; t}$ & $F_{1 ; t}$ & $F_{2 ; t}$ \\
\hline 0 & {$\left[\begin{array}{rr}0 & 1 \\
-1 & 0 \\
1 & 0 \\
0 & 1\end{array}\right]$} & $y_{1}$ & $y_{2}$ & $x_{1}$ & $x_{2}$ & 1 & 1 \\
\hline 1 & {$\left[\begin{array}{rr}0 & -1 \\
1 & 0 \\
1 & 0 \\
0 & -1\end{array}\right]$} & $y_{1}$ & $y_{2}$ & $x_{1}$ & $\frac{x_{1} y_{2}+1}{x_{2}}$ & 1 & $y_{2}+1$ \\
\hline 2 & {$\left[\begin{array}{rr}0 & 1 \\
-1 & 0 \\
-1 & 0 \\
0 & -1\end{array}\right]$} & $\frac{1}{y_{1}}$ & $y_{2}$ & $\frac{x_{1} y_{1} y_{2}+y_{1}+x_{2}}{x_{1} x_{2}}$ & $\frac{x_{1} y_{2}+1}{x_{2}}$ & $y_{1} y_{2}+y_{1}+1$ & $y_{2}+1$ \\
\hline 3 & {$\left[\begin{array}{rr}0 & -1 \\
1 & 0 \\
-1 & 0 \\
-1 & 1\end{array}\right]$} & $\frac{1}{y_{1} y_{2}}$ & $y_{2}$ & $\frac{x_{1} y_{1} y_{2}+y_{1}+x_{2}}{x_{1} x_{2}}$ & $\frac{y_{1}+x_{2}}{x_{1}}$ & $y_{1} y_{2}+y_{1}+1$ & $y_{1}+1$ \\
\hline 4 & {$\left[\begin{array}{rr}0 & 1 \\
-1 & 0 \\
1 & -1 \\
1 & 0\end{array}\right]$} & $y_{1} y_{2}$ & $\frac{1}{y_{1}}$ & $x_{2}$ & $\frac{y_{1}+x_{2}}{x_{1}}$ & 1 & $y_{1}+1$ \\
\hline 5 & {$\left[\begin{array}{rr}0 & -1 \\
1 & 0 \\
0 & 1 \\
1 & 0\end{array}\right]$} & $y_{2}$ & $y_{1}$ & $x_{2}$ & $x_{1}$ & 1 & 1 \\
\hline
\end{tabular}

As explained in $\S 11$, in the special case of cluster algebras of finite type, the polynomials $F_{\ell ; t}$ are closely related to the 'Fibonacci polynomials' of [FZ03b].

We need one more piece of notation in order to state our next theorem. If $F$ is a subtractionfree rational expression over $\mathbb{Q}$ in several variables, $\mathbb{P}$ a semifield, and $u_{1}, \ldots, u_{\ell}$ some elements of $\mathbb{P}$, then we denote by $\left.F\right|_{\mathbb{P}}\left(u_{1}, \ldots, u_{\ell}\right)$ the evaluation of $F$ at $u_{1}, \ldots, u_{\ell}$. This is well defined in view of the universality property of the semifield $\mathbb{Q}_{\mathrm{sf}}\left(u_{1}, \ldots, u_{\ell}\right)$ alluded to in Definition 2.1. For example, if $F\left(u_{1}, u_{2}\right)=u_{1}^{2}-u_{1} u_{2}+u_{2}^{2} \in \mathbb{Q}_{\mathrm{sf}}\left(u_{1}, u_{2}\right)$, and $\mathbb{P}=\operatorname{Trop}\left(y_{1}, y_{2}\right)$, then $\left.F\right|_{\mathbb{P}}\left(y_{1}, y_{2}\right)=$ $\left(y_{1}^{3} \oplus y_{2}^{3}\right) /\left(y_{1} \oplus y_{2}\right)=1$.

Theorem 3.7. Let $\mathcal{A}$ be a cluster algebra over an arbitrary semifield $\mathbb{P}$, with a seed at an initial vertex $t_{0}$ given by (3.1). Then the cluster variables in $\mathcal{A}$ can be expressed as follows:

$$
x_{\ell ; t}=\frac{\left.X_{\ell ; t}^{B^{0} ; t_{0}}\right|_{\mathcal{F}}\left(x_{1}, \ldots, x_{n} ; y_{1}, \ldots, y_{n}\right)}{\left.F_{\ell ; t}^{B^{0} ; t_{0}}\right|_{\mathbb{P}}\left(y_{1}, \ldots, y_{n}\right)} .
$$

Formula (3.5) exhibits the 'separation of additions' phenomenon: the numerator in the righthand side is totally independent of the auxiliary addition $\oplus$, while the denominator does not involve the (ordinary) addition in $\mathcal{F}$. 


\section{S. Fomin And A. Zelevinsky}

Example 3.8. To obtain the expressions for the cluster variables in the last column of Table 1, one needs to replace each + sign in the fifth column of Table 2 by the $\oplus$ sign and then divide each expression in the fourth column of Table 2 by its counterpart in the fifth column.

The proof of Theorem 3.7, to be given later in this section, requires some preparation. Our starting point is the following generalization of [GSV03, Lemma 1.3] and [FG03, (6)].

Proposition 3.9. Let $t \mapsto\left(\mathbf{x}_{t}, \mathbf{y}_{t}, B_{t}\right)$ be a cluster pattern in $\mathcal{F}$, with

$$
\mathbf{x}_{t}=\left(x_{1 ; t}, \ldots, x_{n ; t}\right), \quad \mathbf{y}_{t}=\left(y_{1 ; t}, \ldots, y_{n ; t}\right), \quad B_{t}=\left(b_{i j}^{t}\right)
$$

(as in (2.9)). For $t \in \mathbb{T}_{n}$, let $\hat{\mathbf{y}}_{t}=\left(\hat{y}_{1 ; t}, \ldots, \hat{y}_{n ; t}\right)$ be the $n$-tuple of elements in $\mathcal{F}$ given by

$$
\hat{y}_{j ; t}=y_{j ; t} \prod_{i} x_{i ; t}^{b_{i j}^{t}}
$$

Then $t \mapsto\left(\hat{\mathbf{y}}_{t}, B_{t}\right)$ is a $Y$-pattern in $\mathcal{F}$. In other words, for $t \stackrel{k}{-} t^{\prime}$, we have (cf. (2.3)):

$$
\hat{y}_{j ; t^{\prime}}= \begin{cases}\hat{y}_{k ; t}^{-1} & \text { if } j=k \\ \hat{y}_{j ; t} \hat{y}_{k ; t}^{\left[b_{k j}^{t}\right]_{+}}\left(\hat{y}_{k ; t}+1\right)^{-b_{k j}^{t}} & \text { if } j \neq k\end{cases}
$$

Proof. We denote

$$
x_{i}=x_{i, t}, \quad x_{i}^{\prime}=x_{i, t^{\prime}}, \quad y_{i}=y_{i, t}, \quad y_{i}^{\prime}=y_{i, t^{\prime}}, \quad b_{i j}=b_{i j}^{t}, \quad b_{i j}^{\prime}=b_{i j}^{t^{\prime}}
$$

(so as to match the notation of Definition 2.4) and

$$
\hat{y}_{j}=\hat{y}_{j, t}, \quad \hat{y}_{j}^{\prime}=\hat{y}_{j, t^{\prime}}
$$

With this notation, we get

$$
\hat{y}_{k}^{\prime}=y_{k}^{\prime} \prod_{i \neq k} x_{i}^{b_{i k}^{\prime}}=y_{k}^{-1} \prod_{i \neq k} x_{i}^{-b_{i k}}=\hat{y}_{k}^{-1},
$$

proving the first case in (3.8). To check the second case, note that the exchange relation (2.4) can be rewritten as follows:

$$
x_{k}^{\prime}=\frac{\hat{y}_{k}+1}{y_{k} \oplus 1} x_{k}^{-1} \prod_{i} x_{i}^{\left[-b_{i k}\right]_{+}} .
$$

Using this, for $j \neq k$, we get

$$
\hat{y}_{j}^{\prime}=y_{j}^{\prime}\left(x_{k}^{\prime}\right)^{-b_{k j}} \prod_{i \neq k} x_{i}^{b_{i j}^{\prime}}=y_{j} y_{k}^{\left[b_{k j}\right]_{+}}\left(\hat{y}_{k}+1\right)^{-b_{k j}} x_{k}^{b_{k j}} \prod_{i \neq k} x_{i}^{b_{i j}^{\prime}-\left[-b_{i k}\right]_{+} b_{k j}} .
$$

Applying (2.5), we get

$$
\hat{y}_{j}^{\prime}=\left(y_{j} \prod_{i \neq j} x_{i}^{b_{i j}}\right)\left(y_{k} \prod_{i \neq k} x_{i}^{b_{i k}}\right)^{\left[b_{k j}\right]_{+}}\left(\hat{y}_{k}+1\right)^{-b_{k j}}=\hat{y}_{j} \hat{y}_{k}^{\left[b_{k j}\right]_{+}}\left(\hat{y}_{k}+1\right)^{-b_{k j}},
$$

as required.

To draw some conclusions from Proposition 3.9, we need one more piece of notation.

Definition 3.10 (Rational functions $Y_{\ell, t}$ ). We denote by

$$
Y_{j ; t}=Y_{j ; t}^{B^{0} ; t_{0}} \in \mathbb{Q}_{\mathrm{sf}}\left(y_{1}, \ldots, y_{n}\right)
$$

the components of the $Y$-pattern with values in the universal semifield $\mathbb{Q}_{\mathrm{sf}}\left(y_{1}, \ldots, y_{n}\right)$ (see Definition 2.1), and the initial $Y$-seed $\left(\left(y_{1}, \ldots, y_{n}\right), B^{0}\right)$ at $t_{0}$. 


\section{Cluster algebras IV}

Example 3.11. For a $Y$-pattern of type $A_{2}$ (see Example 2.10), the functions $Y_{j ; t}^{B^{0} ; t_{0}}$ appear in the third column of Table 1 (replace $\oplus$ by + throughout).

By the universality property of the semifield $\mathbb{Q}_{\mathrm{sf}}\left(y_{1}, \ldots, y_{n}\right)$, for any $Y$-pattern $\left(\mathbf{y}_{t}, B_{t}\right)$ with values in an arbitrary semifield $\mathbb{P}$ and the initial $Y$-seed $\left(\left(y_{1}, \ldots, y_{n}\right), B^{0}\right)$ at $t_{0}$, the elements $y_{j ; t} \in \mathbb{P}$ are given by

$$
y_{j ; t}=\left.Y_{j ; t}^{B^{0} ; t_{0}}\right|_{\mathbb{P}}\left(y_{1}, \ldots, y_{n}\right) .
$$

In particular, using the notation of Definition 3.10, Proposition 3.9 can be restated as

$$
\hat{y}_{j ; t}=\left.Y_{j ; t}^{B^{0} ; t_{0}}\right|_{\mathcal{F}}\left(\hat{y}_{1}, \ldots, \hat{y}_{n}\right),
$$

where we abbreviate $\hat{y}_{j}=\hat{y}_{j ; t_{0}}$. Another important specialization of (3.12) is for the patterns of geometric type: using the notation in (2.13) and (2.14), in this case we have

$$
\left.Y_{j ; t}\right|_{\operatorname{Trop}\left(x_{n+1}, \ldots, x_{m}\right)}\left(y_{1}, \ldots, y_{n}\right)=\prod_{i=n+1}^{m} x_{i}^{b_{i j}^{t}},
$$

where $y_{j}=\prod_{i=n+1}^{m} x_{i}^{b_{i j}^{0}}$.

Comparing Definitions 3.3 and 3.10, we note that the rational functions $Y_{j ; t}$ are defined by means of 'general coefficients' while the definition of the polynomials $F_{i ; t}$ involves principal coefficients. Still, both $Y_{j ; t}$ and $F_{i ; t}$ lie in the same semifield $\mathbb{Q}_{s f}\left(y_{1}, \ldots, y_{n}\right)$. In fact, these two families are closely related, as we will now see.

Proposition 3.12. Let $t \stackrel{\ell}{\ell} t^{\prime}$ and $B_{t}=\left(b_{i j}\right)$. Then

$$
F_{\ell ; t^{\prime}}=\frac{Y_{\ell ; t}+1}{\left.\left(Y_{\ell ; t}+1\right)\right|_{\operatorname{Trop}\left(y_{1}, \ldots, y_{n}\right)}} \cdot F_{\ell ; t}^{-1} \prod_{i=1}^{n} F_{i ; t}^{\left[-b_{i \ell}\right]_{+}} .
$$

Proof. The equality (3.15) is a special case of (3.11) for the cluster algebra with principal coefficients under the specialization $x_{1}=\cdots=x_{n}=1$.

Proof of Theorem 3.7. We prove (3.5) by induction on the distance between $t$ and $t_{0}$ in the tree $\mathbb{T}_{n}$. The equality in question is trivial for $t=t_{0}$, both sides of (3.5) being equal to $x_{i}$. So it suffices to show the following: if $t \frac{\ell}{-} t^{\prime}$, and all $x_{i ; t}$ satisfy (3.5) then the same is true for $x_{\ell ; t^{\prime}}$.

Applying (3.11), we obtain

$$
x_{\ell ; t^{\prime}}=\frac{\left.\left(Y_{\ell ; t}+1\right)\right|_{\mathcal{F}}\left(\hat{y}_{1}, \ldots, \hat{y}_{n}\right)}{\left.\left(Y_{\ell ; t}+1\right)\right|_{\mathbb{P}}\left(y_{1}, \ldots, y_{n}\right)} \cdot x_{\ell ; t}^{-1} \prod_{i=1}^{n} x_{i ; t}^{\left[-b_{i \ell}\right]_{+}},
$$

where the elements $\hat{y}_{j}=\hat{y}_{j ; t_{0}} \in \mathcal{F}$ are given by (3.7), and $B=B_{t}$. Applying (3.16) to the cluster algebra with principal coefficients at $t_{0}$, we get

$$
X_{\ell ; t^{\prime}}=\frac{\left(Y_{\ell ; t}+1\right)\left(\hat{y}_{1}, \ldots, \hat{y}_{n}\right)}{\left.\left(Y_{\ell ; t}+1\right)\right|_{\operatorname{Trop}\left(y_{1}, \ldots, y_{n}\right)}\left(y_{1}, \ldots, y_{n}\right)} \cdot X_{\ell ; t}^{-1} \prod_{i=1}^{n} X_{i ; t}^{\left[-b_{i \ell}\right]_{+}}
$$

(note that further specializing all $x_{i}$ to 1 leads to (3.15)). To see that $x_{\ell ; t^{\prime}}$ satisfies (3.5), it suffices to substitute for each $x_{i ; t}$ in the right-hand side of (3.16) its expression given by (3.5), and then to use (3.17) in conjunction with the equality (3.15) evaluated in $\mathbb{P}$.

The following proposition establishes another relation between the rational functions $Y_{j ; t}$ and the polynomials $F_{i ; t}$. 


\section{S. Fomin And A. Zelevinsky}

Proposition 3.13. For any $t \in \mathbb{T}_{n}$ and $j \in[1, n]$, we have

$$
Y_{j ; t}=\left.Y_{j ; t}\right|_{\operatorname{Trop}\left(y_{1}, \ldots, y_{n}\right)} \prod_{i=1}^{n} F_{i ; t}^{b_{i j}},
$$

where $\left(b_{i j}\right)=\left(b_{i j}^{t}\right)=B_{t}$ is the exchange matrix at $t$.

Proof. Apply (3.13) to the cluster algebra with principal coefficients and specialize $x_{1}=\cdots=x_{n}=$ 1 (implying $\hat{y}_{i}=y_{i}$ ). Then (3.18) becomes a special case of (3.7).

Remark 3.14. We note that the monomial $\left.Y_{j ; t}\right|_{\operatorname{Trop}\left(y_{1}, \ldots, y_{n}\right)}$ appearing in (3.18) is nothing but the value of $y_{j ; t}$ in the cluster algebra with principal coefficients at $t_{0}$. For our running example of type $A_{2}$, the monomials $\left.Y_{j ; t}\right|_{\operatorname{Trop}\left(y_{1}, \ldots, y_{n}\right)}$ appear in the third column of Table 2.

Proposition 3.13 has the following useful corollary.

Proposition 3.15. Suppose that $t \in \mathbb{T}_{n}$ and $j \in[1, n]$ are such that $b_{i j}^{t} \geqslant 0$ for all $i$. Then $Y_{j ; t}$ is a Laurent polynomial in $y_{1}, \ldots, y_{n}$.

Proof. This is immediate from (3.18).

\section{Exchange graphs}

We start by recalling some basic definitions from [FZ02, FZ03a].

Definition 4.1 (Seeds). We say that two labeled seeds $\Sigma=(\mathbf{x}, \mathbf{y}, B)$ and $\Sigma^{\prime}=\left(\mathbf{x}^{\prime}, \mathbf{y}^{\prime}, B^{\prime}\right)$ define the same seed if $\Sigma^{\prime}$ is obtained from $\Sigma$ by simultaneous relabeling of the $n$-tuples $\mathbf{x}$ and $\mathbf{y}$ and the corresponding relabeling of the rows and columns of $B$. In other words, seeds are equivalence classes of labeled seeds, where

$$
\Sigma=(\mathbf{x}, \mathbf{y}, B), \quad \mathbf{x}=\left(x_{1}, \ldots, x_{n}\right), \quad \mathbf{y}=\left(y_{1}, \ldots, y_{n}\right), \quad B=\left(b_{i j}\right)
$$

and

$$
\Sigma^{\prime}=\left(\mathbf{x}^{\prime}, \mathbf{y}^{\prime}, B^{\prime}\right), \quad \mathbf{x}^{\prime}=\left(x_{1}^{\prime}, \ldots, x_{n}^{\prime}\right), \quad \mathbf{y}^{\prime}=\left(y_{1}^{\prime}, \ldots, y_{n}^{\prime}\right), \quad B^{\prime}=\left(b_{i j}^{\prime}\right)
$$

are equivalent (denoted $\Sigma \sim \Sigma^{\prime}$ ) if there exists a permutation $\sigma$ of indices $1, \ldots, n$ such that

$$
x_{i}^{\prime}=x_{\sigma(i)}, \quad y_{j}^{\prime}=y_{\sigma(j)}, \quad b_{i, j}^{\prime}=b_{\sigma(i), \sigma(j)}
$$

for all $i$ and $j$. We denote by $[\Sigma]$ the seed represented by a labeled seed $\Sigma$.

For instance, in Example 2.10, the labeled seeds $\Sigma_{0}$ and $\Sigma_{5}$ define the same seed, i.e. $\left[\Sigma_{0}\right]=\left[\Sigma_{5}\right]$.

The (unlabeled) cluster of a seed is an (unordered) $n$-element subset of $\mathcal{F}$ which is a free generating set for $\mathcal{F}$ (cf. Definition 2.3). In view of Definition 2.4, for every element $z$ of the cluster of a seed $[\Sigma]$, there is a well-defined seed $\mu_{z}([\Sigma])=\left[\mu_{k}(\Sigma)\right]$, where $z=x_{k}$ in the labeling provided by $\Sigma$; we say that the seed $\mu_{z}([\Sigma])$ is obtained from $[\Sigma]$ by mutation in direction $z$.

The combinatorics of seed mutations is captured by the exchange graph of a cluster algebra.

Definition 4.2 (Exchange graphs). The exchange graph of a cluster pattern (and of the corresponding cluster algebra) is the $n$-regular (finite or infinite) connected graph whose vertices are the seeds of the cluster pattern and whose edges connect the seeds related by a single mutation.

To illustrate, the exchange graph in Example 2.10 is a 5-cycle.

The exchange graph can be obtained as a quotient of the tree $\mathbb{T}_{n}$ modulo the equivalence relation on vertices defined by setting $t \sim t^{\prime}$ whenever $\Sigma_{t} \sim \Sigma_{t^{\prime}}$ (equivalently, $\left[\Sigma_{t}\right]=\left[\Sigma_{t^{\prime}}\right]$ ). 


\section{Cluster algebras IV}

Each exchange graph is endowed with a canonical (discrete) connection, in the sense of [BGH02, GZ99]. That is, for any two adjacent vertices $[\Sigma]$ and $\left[\Sigma^{\prime}\right]$ in the exchange graph, the $n-1$ edges incident to $[\Sigma]$ but not to $\left[\Sigma^{\prime}\right]$ are in a natural bijection with the edges incident to $\left[\Sigma^{\prime}\right]$ but not to $[\Sigma]$. Specifically, an edge corresponding to the exchange of a cluster variable $z$ from $[\Sigma]$ is matched to the edge corresponding to the exchange of $z$ from $\left[\Sigma^{\prime}\right]$.

Clearly, the exchange graph of a cluster algebra depends only on its underlying $Y$-pattern, which is in turn determined by a $Y$-seed at an arbitrary vertex $t_{0}$ in $\mathbb{T}_{n}$. The following stronger statement was conjectured in [FZ04, Conjecture 4.14(1)].

Conjecture 4.3. The exchange graph of a cluster algebra $\mathcal{A}(\mathbf{x}, \mathbf{y}, B)$ (and the canonical connection on this graph) depends only on the matrix $B$.

Definition 4.4 (Finite type). A cluster algebra $\mathcal{A}$ is of finite type if its exchange graph is finite, that is, $\mathcal{A}$ has finitely many distinct seeds.

Cluster algebras of finite type were classified in [FZ03a]: they correspond to finite root systems. As shown in [FZ03a], the property of a seed $(\mathbf{x}, \mathbf{y}, B)$ to define a cluster algebra of finite type depends only on the matrix $B$.

For cluster algebras of finite type, Conjecture 4.3 was proved in [FZ03a, Theorem 1.13]. It is also known to hold for $n \leqslant 2$ (see [FZ02, Example 7.6]).

As an application of the results in $\S 3$, we obtain a partial result towards Conjecture 4.3.

Definition 4.5 (Coverings of exchange graphs). Let $\mathcal{A}^{\prime}$ and $\mathcal{A}$ be two cluster algebras defined by cluster patterns $t \mapsto \Sigma_{t}=\left(\mathbf{x}_{t}, \mathbf{y}_{t}, B_{t}\right)$ and $t \mapsto \Sigma_{t}^{\prime}=\left(\mathbf{x}_{t}^{\prime}, \mathbf{y}_{t}^{\prime}, B_{t}\right)$ with the same exchange matrices $B_{t}$. We say that the exchange graph of $\mathcal{A}^{\prime}$ covers the exchange graph of $\mathcal{A}$ if the canonical projection of $\mathbb{T}_{n}$ onto the exchange graph of $\mathcal{A}$ factors through the similar projection for $\mathcal{A}^{\prime}$; in other words, $\Sigma_{t_{1}}^{\prime} \sim \Sigma_{t_{2}}^{\prime}$ implies $\Sigma_{t_{1}} \sim \Sigma_{t_{2}}$.

Theorem 4.6. The exchange graph of an arbitrary cluster algebra $\mathcal{A}$ is covered by the exchange graph of the algebra $\mathcal{A}_{\bullet}\left(t_{0}\right)$ which has the same exchange matrices $B_{t}\left(t \in \mathbb{T}_{n}\right)$ as $\mathcal{A}$ and has principal coefficients at some vertex $t_{0}$.

In other words, among all cluster algebras with a given exchange matrix $B^{0}=B_{t_{0}}$, the one with principal coefficients has the 'largest' exchange graph, i.e. one that covers all exchange graphs in this class. It is immediate from Theorem 4.6 that the exchange graph of $\mathcal{A}_{\bullet}\left(t_{0}\right)$ is independent of the choice of $t_{0}$.

It follows from Definition 4.5 that the 'smallest' exchange graph - that is, the one covered by all others - arises in the case of trivial coefficients (all equal to 1), i.e. in the case of a cluster algebra over the one-element semifield $\mathbb{P}=\{1\}$.

Proof of Theorem 4.6. Suppose that in $\mathcal{A}_{\bullet}\left(t_{0}\right)$, the labeled seeds at two vertices $t_{1}$ and $t_{2}$ are equivalent. We need to show that $\Sigma_{t_{1}} \sim \Sigma_{t_{2}}$ in an arbitrary cluster algebra $\mathcal{A}$ with the same exchange matrix $B^{0}$ at $t_{0}$. By definition, we have $X_{i ; t_{2}}^{B^{0} ; t_{0}}=X_{\sigma(i) ; t_{1}}^{B^{0} ; t_{0}},\left.Y_{j ; t_{2}}^{B^{0} ; t_{0}}\right|_{\operatorname{Trop}\left(y_{1}, \ldots, y_{n}\right)}=\left.Y_{\sigma(j) ; t_{1}}^{B^{0} ; t_{0}}\right|_{\operatorname{Trop}\left(y_{1}, \ldots, y_{n}\right)}$, and $b_{i j}^{t_{2}}=b_{\sigma(i), \sigma(j)}^{t_{1}}$ for some permutation $\sigma$ of $[1, n]$. This implies, in particular, that $F_{i ; t_{2}}^{B^{0} ; t_{0}}=F_{\sigma(i) ; t_{1}}^{B^{0} ; t_{0}}$. We need to show that in $\mathcal{A}$, we have $x_{i ; t_{2}}=x_{\sigma(i) ; t_{1}}$ and $y_{j ; t_{2}}=y_{\sigma(j) ; t_{1}}$ for all $i$ and $j$. The former equality follows at once from (3.5); the latter follows from the equality $Y_{j ; t_{2}}^{B^{0} ; t_{0}}=Y_{\sigma(j) ; t_{1}}^{B^{0} ; t_{0}}$, which is a consequence of (3.18).

The following conjecture asserts that the exchange graph of $\mathcal{A}_{\bullet}\left(t_{0}\right)$ has a purely combinatorial description in terms of matrix mutations. 


\section{S. Fomin And A. Zelevinsky}

CONJECTURe 4.7. Let $\left(\tilde{B}_{t}\right)_{t \in \mathbb{T}_{n}}$ be a family of $2 n \times n$ extended exchange matrices associated with the algebra $\mathcal{A}_{\bullet}\left(t_{0}\right)$ (see Remark 3.2). Then the labeled seeds at two vertices $t$ and $t^{\prime}$ define the same seed of $\mathcal{A}_{\bullet}\left(t_{0}\right)$ if and only if $\tilde{B}_{t^{\prime}}$ is obtained from $\tilde{B}_{t}$ by a simultaneous permutation of rows and columns of the principal part $B_{t}$. In other words, in the cluster algebra $\mathcal{A}_{\bullet}\left(t_{0}\right)$, the cluster of a seed $(\mathbf{x}, \mathbf{y}, B)$ is uniquely determined by the corresponding $Y$-seed $(\mathbf{y}, B)$.

Conjecture 4.7 is not yet checked even for algebras of finite type. Note that it implies the following combinatorial characterization of finite type exchange matrices.

We say that a matrix $\tilde{B}$ has finite mutation type if its mutation equivalence class is finite, i.e. only finitely many matrices can be obtained from $\tilde{B}$ by repeated matrix mutations.

Conjecture 4.8. An exchange matrix $B_{t_{0}}$ gives rise to cluster algebras of finite type if and only if the corresponding $2 n \times n$ matrix $\tilde{B}_{t_{0}}$ defined as in Remark 3.2 is of finite mutation type.

It is the 'if' part of Conjecture 4.8 that still remains open. At the moment we can only prove the following weaker statement.

Proposition 4.9. An exchange matrix $B$ gives rise to cluster algebras of finite type if and only if any integer matrix $\tilde{B}$ having $B$ as its principal part is of finite mutation type.

Again, only the 'if' part needs a proof. It will be given in $\S 10$.

\section{Properties of $\boldsymbol{F}$-polynomials}

In this section we discuss some properties (partly conjectural) of the polynomials $F_{\ell ; t}=F_{\ell ; t}^{B^{0} ; t_{0}} \in$ $\mathbb{Z}\left[y_{1}, \ldots, y_{n}\right]$ given by $(3.3)$. We start by writing down, in a closed form, the recurrence relations for these polynomials.

Proposition 5.1. Let $t \mapsto \tilde{B}_{t}=\left(b_{i j}^{t}\right)\left(t \in \mathbb{T}_{n}\right)$ be the family of $2 n \times n$ matrices associated with the cluster algebra $\mathcal{A}_{\bullet}\left(B_{t_{0}}\right)$. That is, $\tilde{B}_{t_{0}}$ is the matrix in Remark 3.2 , and $\tilde{B}_{t^{\prime}}=\mu_{k}\left(\tilde{B}_{t}\right)$ whenever $t \stackrel{k}{t} t^{\prime}$. Then the polynomials $F_{\ell ; t}=F_{\ell ; t}^{B_{t_{0}} ; t_{0}}\left(y_{1}, \ldots, y_{n}\right)$ are uniquely determined by the initial conditions

$$
F_{\ell ; t_{0}}=1 \quad(\ell=1, \ldots, n),
$$

together with the recurrence relations

$$
\begin{gathered}
F_{\ell ; t^{\prime}}=F_{\ell ; t} \quad \text { for } \ell \neq k ; \\
F_{k ; t^{\prime}}=\frac{\prod_{j=1}^{n} y_{j}^{\left[b_{n+j, k}^{t}\right]_{+}} \prod_{i=1}^{n} F_{i ; t}^{\left[b_{i k}^{t}\right]_{+}}+\prod_{j=1}^{n} y_{j}^{\left[-b_{n+j, k}^{t}\right]_{+}} \prod_{i=1}^{n} F_{i ; t}^{\left[-b_{i k}^{t}\right]_{+}}}{F_{k ; t}},
\end{gathered}
$$

for every edge $t \stackrel{k}{-} t^{\prime}$ such that $t$ lies on the (unique) path from $t_{0}$ to $t^{\prime}$ in $\mathbb{T}_{n}$.

Proof. The relations (5.2) and (5.3) are immediate consequences of the exchange relations in the cluster algebra with principal coefficients at $t_{0}$, which are a special case of $(2.15)$ :

$$
X_{k ; t} X_{k ; t^{\prime}}=\prod_{j=1}^{n} y_{j}^{\left[b_{n+j, k}^{t}\right]+} \prod_{i=1}^{n} X_{i ; t}^{\left[b_{i k}^{t}\right]+}+\prod_{j=1}^{n} y_{j}^{\left[-b_{n+j, k}^{t}\right]_{+}} \prod_{i=1}^{n} X_{i ; t}^{\left[-b_{i k}^{t}\right]_{+}} .
$$

To deduce (5.3) from (5.4), specialize all initial cluster variables to 1.

We next draw some consequences from (3.5).

Proposition 5.2. Each of the polynomials $F_{\ell ; t}\left(y_{1}, \ldots, y_{n}\right)$ is not divisible by any $y_{j}$. 


\section{Cluster algebras IV}

Proof. Applying (3.5) to the algebra with principal coefficients at $t_{0}$, we conclude that the denominator on the right-hand side becomes equal to 1 , that is,

$$
\left.F_{\ell ; t}^{B^{0} ; t_{0}}\right|_{\operatorname{Trop}\left(y_{1}, \ldots, y_{n}\right)}=1 \text {, }
$$

which is precisely our statement.

Our next result relates the polynomials $F_{\ell ; t}^{B^{0} ; t_{0}}$ and $F_{\ell ; t}^{-B^{0} ; t_{0}}$.

Proposition 5.3. The polynomials $F_{\ell ; t}^{B^{0} ; t_{0}}$ and $F_{\ell ; t}^{-B^{0} ; t_{0}}$ are related by

$$
F_{\ell ; t}^{B^{0} ; t_{0}}\left(y_{1}, \ldots, y_{n}\right)=\frac{F_{\ell ; t}^{-B^{0} ; t_{0}}\left(y_{1}^{-1}, \ldots, y_{n}^{-1}\right)}{\left.F_{\ell ; t}^{-B^{0} ; t_{0}}\right|_{\operatorname{Trop}\left(y_{1}, \ldots, y_{n}\right)}\left(y_{1}^{-1}, \ldots, y_{n}^{-1}\right)} .
$$

Proof. Using the notation in Definition 3.3, let us introduce the cluster algebra $\mathcal{A}^{\prime}$ which shares with $\mathcal{A}=\mathcal{A}_{\bullet}\left(B^{0}\right)$ the same ambient field, the same coefficient semifield $\mathbb{P}=\operatorname{Trop}\left(y_{1}, \ldots, y_{n}\right)$, and the same initial cluster $\mathbf{x}_{t_{0}}=\left(x_{1}, \ldots, x_{n}\right)$, but has the initial $Y$-seed $\left(\mathbf{y}_{t_{0}}^{\prime}, B_{t_{0}}^{\prime}\right)$ given by $\mathbf{y}_{t_{0}}^{\prime}=$ $\left(y_{1}^{-1}, \ldots, y_{n}^{-1}\right), B_{t_{0}}^{\prime}=-B^{0}$. Comparing the definitions, we see that all of the cluster variables $x_{\ell ; t}$ in $\mathcal{A}^{\prime}$ are the same as in $\mathcal{A}$. Applying (3.5), we get

$$
X_{\ell ; t}^{B^{0} ; t_{0}}\left(x_{1}, \ldots, x_{n} ; y_{1}, \ldots, y_{n}\right)=\frac{X_{\ell ; t}^{-B^{0} ; t_{0}}\left(x_{1}, \ldots, x_{n} ; y_{1}^{-1}, \ldots, y_{n}^{-1}\right)}{\left.F_{\ell ; t}^{-B^{0} ; t_{0}}\right|_{\operatorname{Trop}\left(y_{1}, \ldots, y_{n}\right)}\left(y_{1}^{-1}, \ldots, y_{n}^{-1}\right)},
$$

which implies (5.6) by specializing all $x_{i}$ to 1 .

We now present a tantalizing conjecture which is a strengthening of Proposition 5.2.

ConjeCture 5.4. Each polynomial $F_{\ell ; t}^{B^{0} ; t_{0}}\left(y_{1}, \ldots, y_{n}\right)$ has constant term 1.

In view of Proposition 5.3, Conjecture 5.4 is equivalent to the following.

CONJECTURE 5.5. Each polynomial $F_{\ell ; t}^{B^{0} ; t_{0}}\left(y_{1}, \ldots, y_{n}\right)$ has a unique monomial of maximal degree. Furthermore, this monomial has coefficient 1, and it is divisible by all of the other occurring monomials.

Some special cases of Conjecture 5.4 are proved in subsequent sections. Here we give some equivalent reformulations.

Proposition 5.6. For a given initial seed, the following are equivalent.

(i) Each polynomial $F_{\ell ; t}\left(y_{1}, \ldots, y_{n}\right)$ has constant term 1.

(ii) Each tropical evaluation $\left.Y_{\ell ; t}\right|_{\operatorname{Trop}\left(y_{1}, \ldots, y_{n}\right)}$ is a monomial in $y_{1}, \ldots, y_{n}$ which has either all exponents non-negative or all exponents non-positive.

(iii) In the corresponding cluster algebra with principal coefficients, in each exchange relation (2.4)/(2.8), exactly one of the coefficients $p_{k}^{ \pm}$is equal to 1 .

Proof. In the notation of Proposition 5.1, we have

$$
\left.Y_{\ell ; t}\right|_{\operatorname{Trop}\left(y_{1}, \ldots, y_{n}\right)}=\prod_{j=1}^{n} y_{j}^{b_{n+j, \ell}^{t}}
$$

this is a special case of (3.14). Thus (ii) can be rephrased as follows.

(ii') For any $\ell$ and $t$, the integer vector $\mathbf{c}_{\ell ; t}=\left(b_{n+1, \ell}^{t}, \ldots, b_{2 n, \ell}^{t}\right)$ has either all components nonnegative or all components non-positive. 


\section{S. Fomin And A. Zelevinsky}

In view of (5.4), the coefficients in the exchange relations are of the form

$$
p^{ \pm}=\prod_{j=1}^{n} y_{j}^{\left[ \pm b_{n+j, \ell}^{t}\right]_{+}} .
$$

Hence, (iii) $\Rightarrow\left(\mathrm{ii}^{\prime}\right)$. To prove the converse implication (ii' $) \Rightarrow(\mathrm{iii})$, it remains to show that (ii') implies that for any $\ell$ and $t$, we have $\mathbf{c}_{\ell ; t} \neq 0$. Recall that $\tilde{B}_{t^{\prime}}=\mu_{k}\left(\tilde{B}_{t}\right)$ whenever $t \stackrel{k}{-} t^{\prime}$. Under the assumption (ii'), the matrix mutation rule (2.2) can be rewritten as follows:

$$
\mathbf{c}_{\ell ; t^{\prime}}= \begin{cases}-\mathbf{c}_{\ell ; t} & \text { if } \ell=k ; \\ \mathbf{c}_{\ell ; t}+\left[b_{k \ell}\right]_{+} \mathbf{c}_{k ; t} & \text { if } b_{n+i, k}^{t} \geqslant 0 \text { for all } i \\ \mathbf{c}_{\ell ; t}+\left[-b_{k \ell}\right]_{+} \mathbf{c}_{k ; t} & \text { if } b_{n+i, k}^{t} \leqslant 0 \text { for all } i .\end{cases}
$$

It follows that, as long as (ii') holds, matrix mutations act by invertible linear transformations on the bottom part of $\tilde{B}_{t}$. The latter must therefore have full rank, and so has no zero columns.

It remains to demonstrate that (i) $\Leftrightarrow$ (iii). The implication (i) $\Rightarrow$ (iii) is immediate from (5.3): if all participating $F$-polynomials take value 1 at $y_{1}=\cdots=y_{n}=0$, then exactly one of the coefficients on the right must be equal to 1 . The converse implication (iii) $\Rightarrow$ (i) also follows from (5.3) by induction on the distance from $t$ to $t_{0}$ in $\mathbb{T}_{n}$.

\section{Principal $\mathbb{Z}^{n}$-gradings}

In this section we introduce and study a natural $\mathbb{Z}^{n}$-grading in a cluster algebra with principal coefficients. As an application, we obtain, in Corollary 6.3 below, a refinement of Theorem 3.7.

Proposition 6.1. Every Laurent polynomial $X_{\ell ; t}^{B^{0} ; t_{0}}$ is homogeneous with respect to the $\mathbb{Z}^{n}$-grading in $\mathbb{Z}\left[x_{1}^{ \pm 1}, \ldots, x_{n}^{ \pm 1} ; y_{1}, \ldots, y_{n}\right]$ given by

$$
\operatorname{deg}\left(x_{i}\right)=\mathbf{e}_{i}, \quad \operatorname{deg}\left(y_{j}\right)=-\mathbf{b}_{j}^{0},
$$

where $\mathbf{e}_{1}, \ldots, \mathbf{e}_{n}$ are the standard basis (column) vectors in $\mathbb{Z}^{n}$, and $\mathbf{b}_{j}^{0}=\sum_{i} b_{i j}^{0} \mathbf{e}_{i}$ is the $j$ th column of $B^{0}$.

Proof. Let $\hat{y}_{1}, \ldots, \hat{y}_{n}$ be given by

$$
\hat{y}_{j}=y_{j} \prod_{i} x_{i}^{b_{i j}^{0}}
$$

(cf. (3.7)). In view of (6.1), we have

$$
\operatorname{deg}\left(\hat{y}_{j}\right)=0
$$

Now let us apply (3.17). Using induction on the distance from $t_{0}$ in $\mathbb{T}_{n}$, it suffices to show that the element $X_{\ell ; t^{\prime}}$ on the left-hand side is homogeneous provided that all of the elements $X_{i ; t}$ on the right-hand side are also homogeneous. In other words, it remains to show the homogeneity of

$$
\frac{\left(Y_{\ell ; t}+1\right)\left(\hat{y}_{1}, \ldots, \hat{y}_{n}\right)}{\left.\left(Y_{\ell ; t}+1\right)\right|_{\operatorname{Trop}\left(y_{1}, \ldots, y_{n}\right)}\left(y_{1}, \ldots, y_{n}\right)} \text {. }
$$

The numerator $\left(Y_{\ell ; t}+1\right)\left(\hat{y}_{1}, \ldots, \hat{y}_{n}\right)$ is homogeneous of degree 0 by $(6.3)$. The denominator is a Laurent monomial in $y_{1}, \ldots, y_{n}$, so it is homogeneous as well.

Proposition 6.1 can be restated as follows (cf. Definitions 3.1 and 3.3).

Corollary 6.2. Under the $\mathbb{Z}^{n}$-grading given by (6.1), the cluster algebra $\mathcal{A}_{\bullet}\left(B_{t_{0}}\right)$ is a $\mathbb{Z}^{n}$-graded subalgebra of $\mathbb{Z}\left[x_{1}^{ \pm 1}, \ldots, x_{n}^{ \pm 1} ; y_{1}, \ldots, y_{n}\right]$. All cluster variables in $\mathcal{A} \bullet\left(B_{t_{0}}\right)$ are homogeneous elements. 


\section{Cluster algebras IV}

We use the notation

$$
\mathbf{g}_{\ell ; t}=\mathbf{g}_{\ell ; t}^{B^{0} ; t_{0}}=\left[\begin{array}{c}
g_{1} \\
\vdots \\
g_{n}
\end{array}\right]=\operatorname{deg}\left(X_{\ell ; t}^{B^{0} ; t_{0}}\right) \in \mathbb{Z}^{n}
$$

(see (6.1)) for the (multi-)degrees of the cluster variables in $\mathcal{A}_{\bullet}\left(B_{t_{0}}\right)$, and refer to the integer vectors thus obtained as $\mathbf{g}$-vectors.

We can now refine the 'separation of additions' result in Theorem 3.7, as follows.

Corollary 6.3. Cluster variables in an arbitrary cluster algebra $\mathcal{A}=\mathcal{A}\left(\mathbf{x}_{0}, \mathbf{y}_{0}, B^{0}\right)$ can be expressed in terms of the initial seed (3.1) by the formula

$$
x_{\ell ; t}=\frac{\left.F_{\ell ; t}^{B^{0} ; t_{0}}\right|_{\mathcal{F}}\left(\hat{y}_{1}, \ldots, \hat{y}_{n}\right)}{\left.F_{\ell ; t}^{B^{0} ; t_{0}}\right|_{\mathbb{P}}\left(y_{1}, \ldots, y_{n}\right)} x_{1}^{g_{1}} \cdots x_{n}^{g_{n}},
$$

where we use the notation (6.2) and (6.4).

Proof. Proposition 6.1 can be restated as follows:

$$
X_{\ell ; t}^{B^{0} ; t_{0}}\left(\gamma_{1} x_{1}, \ldots, \gamma_{n} x_{n} ; \ldots, \prod_{k} \gamma_{k}^{-b_{k j}^{0}} y_{j}, \ldots\right)=\left(\prod_{k} \gamma_{k}^{g_{k}}\right) X_{\ell ; t}^{B^{0} ; t_{0}}\left(x_{1}, \ldots, x_{n} ; y_{1}, \ldots, y_{n}\right)
$$

for any rational functions $\gamma_{1}, \ldots, \gamma_{n}$. Applying (6.6) with $\gamma_{k}=x_{k}^{-1}$, we obtain

$$
X_{\ell ; t}^{B^{0} ; t_{0}}\left(x_{1}, \ldots, x_{n} ; y_{1}, \ldots, y_{n}\right)=x_{1}^{g_{1}} \cdots x_{n}^{g_{n}} F_{\ell ; t}^{B^{0} ; t_{0}}\left(\hat{y}_{1}, \ldots, \hat{y}_{n}\right) .
$$

Substituting (6.7) into (3.5) yields (6.5).

Example 6.4. In the special case of type $A_{2}$ (Examples 2.10 and 3.4 ), the $\mathbf{g}$-vectors $\mathbf{g}_{\ell ; t}=\mathbf{g}_{\ell ; t}^{B^{0} ; t_{0}}$ can be read off the fourth column of Table 2, by taking the monomials not involving the $y$ variables and recording their exponents. The results are shown in the second column of Table 3, where we use the shorthand

$$
x^{\mathbf{g}_{\ell ; t}}=\prod_{i} x_{i}^{g_{i}}
$$

Substituting $\hat{y}_{1}=y_{1} x_{2}^{-1}, \hat{y}_{2}=y_{2} x_{1}$ into the fifth column of Table 2, we obtain the Laurent polynomials $F_{\ell ; t}\left(\hat{y}_{1}, \hat{y}_{2}\right)$ shown in the third column of Table 3 . The fourth column of this table lists the polynomials $\left.F_{\ell ; t}\right|_{\mathbb{P}}\left(y_{1}, y_{2}\right)$. Thus, Table 3 provides all of the ingredients for the right-hand side of (6.5) for the cluster algebra under consideration. The resulting formulas for the cluster variables match those in the fourth column of Table 1.

Remark 6.5 (The $+/ \oplus$-symmetry). The formula (6.5) exhibits certain symmetry between the ordinary addition in $\mathcal{F}$ and the auxiliary addition in $\mathbb{P}$. To make this symmetry more transparent, we note that this formula (as well as many other formulas in this paper) extends to the following more general setup. Let $M$ be an abelian multiplicative group, with two distinguished subgroups $\mathbb{P}$ and $\hat{\mathbb{P}}$, each of which is endowed with a semifield structure, which we write as $(\mathbb{P}, \oplus)$ and $(\hat{\mathbb{P}},+)$, respectively. (In our current setup, think of $M$ as the multiplicative group of $\mathcal{F}$, with $\mathbb{P}$ the coefficient semifield, and $\hat{\mathbb{P}}$ the subsemifield in $\mathcal{F}$ generated by all of the elements $\hat{y}_{j ; t}$ given by (3.7).) We can now define a (labeled) $(M, \mathbb{P}, \hat{\mathbb{P}})$-seed as follows:

$$
\Sigma=\left(\left(x_{1}, \ldots, x_{n} ; y_{1}, \ldots, y_{n} ; \hat{y}_{1}, \ldots, \hat{y}_{n}\right), B^{0}\right) \quad\left(x_{j} \in M, y_{j} \in \mathbb{P}, \hat{y}_{j} \in \hat{\mathbb{P}}\right),
$$

where $B^{0}$ is a skew-symmetrizable integer $n \times n$ matrix (see Definition 2.3), and the elements $x_{j}, y_{j}$ and $\hat{y}_{j}$ satisfy $(6.2)$. Then, for any $k=1, \ldots, n$, the $(M, \mathbb{P}, \hat{\mathbb{P}})$-seed mutation in direction $k$ 


\section{S. Fomin And A. Zelevinsky}

TABLE 3. g-vectors and Laurent polynomials $F_{\ell ; t}\left(\hat{y}_{1}, \hat{y}_{2}\right)$ in type $A_{2}$.

\begin{tabular}{c|cc|cc|cc}
\hline$t$ & $x^{\mathbf{g}_{1 ; t}}$ & $x^{\mathbf{g}_{2 ; t}}$ & $F_{1 ; t}\left(\hat{y}_{1}, \hat{y}_{2}\right)$ & $F_{2 ; t}\left(\hat{y}_{1}, \hat{y}_{2}\right)$ & $\left.F_{1 ; t}\right|_{\mathbb{P}}$ & $F_{2 ; t} \mid \mathbb{P}$ \\
\hline 0 & $x_{1}$ & $x_{2}$ & 1 & 1 & 1 & 1 \\
1 & $x_{1}$ & $\frac{1}{x_{2}}$ & 1 & $x_{1} y_{2}+1$ & 1 & $y_{2} \oplus 1$ \\
2 & $\frac{1}{x_{1}}$ & $\frac{1}{x_{2}}$ & $\frac{x_{1} y_{1} y_{2}+y_{1}+x_{2}}{x_{2}}$ & $x_{1} y_{2}+1$ & $y_{1} y_{2} \oplus y_{1} \oplus 1$ & $y_{2} \oplus 1$ \\
3 & $\frac{1}{x_{1}}$ & $\frac{x_{2}}{x_{1}}$ & $\frac{x_{1} y_{1} y_{2}+y_{1}+x_{2}}{x_{2}}$ & $\frac{y_{1}+x_{2}}{x_{2}}$ & $y_{1} y_{2} \oplus y_{1} \oplus 1$ & $y_{1} \oplus 1$ \\
4 & $x_{2}$ & $\frac{x_{2}}{x_{1}}$ & 1 & $\frac{y_{1}+x_{2}}{x_{2}}$ & 1 & $y_{1} \oplus 1$ \\
5 & $x_{2}$ & $x_{1}$ & 1 & 1 & 1 & 1 \\
\hline
\end{tabular}

transforms $\Sigma$ into

$$
\Sigma^{\prime}=\mu_{k}(\Sigma)=\left(\left(x_{1}^{\prime}, \ldots, x_{n}^{\prime} ; y_{1}^{\prime}, \ldots, y_{n}^{\prime} ; \hat{y}_{1}^{\prime}, \ldots, \hat{y}_{n}^{\prime}\right), B^{1}\right)
$$

given as follows:

- $B^{1}$ is obtained from $B^{0}$ by the matrix mutation in direction $k$ (see $(2.2)$ );

- the tuple $\left(y_{1}^{\prime}, \ldots, y_{n}^{\prime}\right)$ (respectively, $\left.\left(\hat{y}_{1}^{\prime}, \ldots, \hat{y}_{n}^{\prime}\right)\right)$ is obtained from $\left(y_{1}, \ldots, y_{n}\right)$ (respectively, $\left.\left(\hat{y}_{1}, \ldots, \hat{y}_{n}\right)\right)$ by the $Y$-seed mutation $(2.3)$ in the semifield $\mathbb{P}$ (respectively, $\hat{\mathbb{P}}$ );

- $x_{i}^{\prime}=x_{i}$ for $i \neq k$, and $x_{k}^{\prime}$ is given by (3.11) with $b_{i k}=b_{i k}^{0}$.

The fact that $\Sigma^{\prime}$ is indeed a $(M, \mathbb{P}, \hat{\mathbb{P}})$-seed is shown by calculations in the proof of Proposition 3.9.

Following Definition 2.9 , we can define a $(M, \mathbb{P}, \hat{\mathbb{P}})$-pattern as an assignment of an $(M, \mathbb{P}, \hat{\mathbb{P}})$-seed $\Sigma_{t}$ to every vertex $t \in \mathbb{T}_{n}$, such that the seeds assigned to the endpoints of any edge $t \frac{k}{k} t^{\prime}$ are obtained from each other by the mutation in direction $k$. If the seed $\Sigma_{t_{0}}$ is given by (6.8), and $\Sigma_{t}$ is written as

$$
\Sigma_{t}=\left(\left(x_{1 ; t}, \ldots, x_{n ; t} ; y_{1 ; t}, \ldots, y_{n ; t} ; \hat{y}_{1 ; t}, \ldots, \hat{y}_{n ; t}\right), B_{t}\right),
$$

then the elements of $\Sigma_{t}$ are given by the expressions (3.12), (3.13), and (6.5), where in the last two formulas the evaluation in $\mathcal{F}$ is replaced by that in $\hat{\mathbb{P}}$. These expressions imply the following $+/ \oplus$-duality: every $(M, \mathbb{P}, \hat{\mathbb{P}})$-pattern gives rise to a $(M, \hat{\mathbb{P}}, \mathbb{P})$-pattern obtained by replacing each $(M, \mathbb{P}, \hat{\mathbb{P}})$-seed $(6.9)$ with the $(M, \hat{\mathbb{P}}, \mathbb{P})$-seed

$$
\left(\left(x_{1 ; t}^{-1}, \ldots, x_{n ; t}^{-1} ; \hat{y}_{1 ; t}, \ldots, \hat{y}_{n ; t} ; y_{1 ; t}, \ldots, y_{n ; t}\right), B_{t}\right) .
$$

Returning to cluster algebras, we note that, in practical terms, using the formula (6.5) to compute some cluster variable $x_{\ell ; t}$ in terms of an initial cluster at a vertex $t_{0}$ requires calculating the polynomial $F_{\ell ; t}^{B^{0} ; t_{0}}\left(y_{1}, \ldots, y_{n}\right)$ and the $\mathbf{g}$-vector $\mathbf{g}_{\ell ; t}^{B^{0} ; t_{0}}$. The polynomial $F_{\ell: t}^{B^{0} ; t_{0}}\left(y_{1}, \ldots, y_{n}\right)$ can be calculated recursively by Proposition 5.1. As to computation of the g-vector, it can be accomplished using recurrences (6.11) and (6.12) below.

Proposition 6.6. In the notation of Proposition 5.1, the $\mathbf{g}$-vectors $\mathbf{g}_{\ell ; t}=\mathbf{g}_{\ell ; t}^{B^{0} ; t_{0}}$ are uniquely determined by the initial conditions

$$
\mathbf{g}_{\ell ; t_{0}}=\mathbf{e}_{\ell} \quad(\ell=1, \ldots, n)
$$




\section{Cluster algebras IV}

together with the recurrence relations

$$
\begin{gathered}
\mathbf{g}_{\ell ; t^{\prime}}=\mathbf{g}_{\ell ; t} \quad \text { for } \ell \neq k \\
\mathbf{g}_{k ; t^{\prime}}=-\mathbf{g}_{k ; t}+\sum_{i=1}^{n}\left[b_{i k}^{t}\right]_{+} \mathbf{g}_{i ; t}-\sum_{j=1}^{n}\left[b_{n+j, k}^{t}\right]_{+} \mathbf{b}_{j}^{0},
\end{gathered}
$$

where the (unique) path from $t_{0}$ to $t^{\prime}$ in $\mathbb{T}_{n}$ ends with the edge $t \stackrel{k}{-} t^{\prime}$, and $\mathbf{b}_{j}^{0}$ stands for the $j$ th column of $B^{0}$.

Proof. The relations (6.11) and (6.12) follow from (5.4) by equating the degree of the product $X_{k ; t} X_{k ; t^{\prime}}$ with that of the first term on the right.

Note that (6.12) can be replaced by the relation

$$
\mathbf{g}_{k ; t^{\prime}}=-\mathbf{g}_{k ; t}+\sum_{i=1}^{n}\left[-b_{i k}^{t}\right]_{+} \mathbf{g}_{i ; t}-\sum_{j=1}^{n}\left[-b_{n+j, k}^{t}\right]_{+} \mathbf{b}_{j}^{0},
$$

obtained by taking the second term on the right in (5.4) instead of the first term. The fact that (6.12) and (6.13) agree is equivalent to the identity

$$
\sum_{i=1}^{n} b_{i k}^{t} \mathbf{g}_{i ; t}=\sum_{j=1}^{n} b_{n+j, k}^{t} \mathbf{b}_{j}^{0}
$$

which is simply another way of saying that the element $Y_{k ; t}\left(\hat{y}_{1}, \ldots, \hat{y}_{n}\right)$ is homogeneous of degree 0 (see (6.3) and (3.13)).

Example 6.7. The results of g-vector calculations based on (6.11) and (6.12) for a cluster algebra of type $A_{2}$ (Examples 2.10 and 3.4) are shown in the third column of Table 4.

We now present an alternative recursive description of the $\mathbf{g}$-vectors $\mathbf{g}_{\ell ; t}^{t_{0}}$ that is based on fixing $\ell$ and $t$ and allowing the initial vertex $t_{0}$ to vary.

Proposition 6.8. Let $t_{0} \stackrel{k}{-} t_{1}$, and $B^{1}=\mu_{k}\left(B^{0}\right)$. Then the $\mathbf{g}$-vectors

$$
\mathbf{g}_{\ell ; t}^{B^{0} ; t_{0}}=\left[\begin{array}{c}
g_{1} \\
\vdots \\
g_{n}
\end{array}\right] \quad \text { and } \quad \mathbf{g}_{\ell ; t}^{B^{1} ; t_{1}}=\left[\begin{array}{c}
g_{1}^{\prime} \\
\vdots \\
g_{n}^{\prime}
\end{array}\right]
$$

are related as follows:

$$
g_{i}= \begin{cases}-g_{k}^{\prime} & \text { if } i=k ; \\ g_{i}^{\prime}+\left[-b_{i k}^{0}\right]_{+} g_{k}^{\prime}+b_{i k}^{0} h_{k}^{\prime} & \text { if } i \neq k,\end{cases}
$$

where the integer $h_{k}^{\prime}$ is defined by

$$
u^{h_{k}^{\prime}}=\left.F_{\ell, t}^{B^{1} ; t_{1}}\right|_{\operatorname{Trop}(u)}\left(u^{\left[b_{k 1}^{0}\right]_{+}}, \ldots, u^{-1}, \ldots, u^{\left[b_{k n}^{0}\right]_{+}}\right) \quad\left(u^{-1} \text { in the } k \text { th position }\right) .
$$

Proof. We start by preparing some needed notation. We will work in the cluster algebra $\mathcal{A}=\mathcal{A}_{\bullet}\left(B^{0}\right)$ with principal coefficients at a vertex $t_{0}$, and adopt the notation in Definition 3.3. Thus, the seed $\Sigma_{t_{0}}=\left(\mathbf{x}_{t_{0}}, \mathbf{y}_{t_{0}}, B_{t_{0}}\right)$ is given by (3.1), and the coefficient semifield is $\mathbb{P}=\operatorname{Trop}\left(y_{1}, \ldots, y_{n}\right)$. Let $\Sigma_{t_{1}}=$ $\left(\mathbf{x}_{t_{1}}, \mathbf{y}_{t_{1}}, B_{t_{1}}\right)$ be the adjacent seed at $t_{1}$ obtained from $\Sigma_{t_{0}}$ by the mutation in direction $k$ given by $(2.2)-(2.4)$. In more detail, $\Sigma_{t_{1}}$ is given as follows. 


\section{S. Fomin And A. Zelevinsky}

TABLE 4. g-vectors and denominators in type $A_{2}$.

\begin{tabular}{|c|c|c|c|c|c|}
\hline$t$ & $\tilde{B}_{t}$ & $\mathbf{g}_{1 ; t}$ & $\mathbf{g}_{2 ; t}$ & $\mathbf{d}_{1 ; t}$ & $\mathbf{d}_{2 ; t}$ \\
\hline 0 & {$\left[\begin{array}{rr}0 & 1 \\
-1 & 0 \\
1 & 0 \\
0 & 1\end{array}\right]$} & {$\left[\begin{array}{l}1 \\
0\end{array}\right]$} & {$\left[\begin{array}{l}0 \\
1\end{array}\right]$} & {$\left[\begin{array}{r}-1 \\
0\end{array}\right]$} & {$\left[\begin{array}{r}0 \\
-1\end{array}\right]$} \\
\hline 1 & {$\left[\begin{array}{rr}0 & -1 \\
1 & 0 \\
1 & 0 \\
0 & -1\end{array}\right]$} & {$\left[\begin{array}{l}1 \\
0\end{array}\right]$} & {$\left[\begin{array}{r}0 \\
-1\end{array}\right]$} & {$\left[\begin{array}{r}-1 \\
0\end{array}\right]$} & {$\left[\begin{array}{l}0 \\
1\end{array}\right]$} \\
\hline 2 & {$\left[\begin{array}{rr}0 & 1 \\
-1 & 0 \\
-1 & 0 \\
0 & -1\end{array}\right]$} & {$\left[\begin{array}{r}-1 \\
0\end{array}\right]$} & {$\left[\begin{array}{r}0 \\
-1\end{array}\right]$} & {$\left[\begin{array}{l}1 \\
1\end{array}\right]$} & {$\left[\begin{array}{l}0 \\
1\end{array}\right]$} \\
\hline 3 & {$\left[\begin{array}{rr}0 & -1 \\
1 & 0 \\
-1 & 0 \\
-1 & 1\end{array}\right]$} & {$\left[\begin{array}{r}-1 \\
0\end{array}\right]$} & {$\left[\begin{array}{r}-1 \\
1\end{array}\right]$} & {$\left[\begin{array}{l}1 \\
1\end{array}\right]$} & {$\left[\begin{array}{l}1 \\
0\end{array}\right]$} \\
\hline 4 & {$\left[\begin{array}{rr}0 & 1 \\
-1 & 0 \\
1 & -1 \\
1 & 0\end{array}\right]$} & {$\left[\begin{array}{l}0 \\
1\end{array}\right]$} & {$\left[\begin{array}{r}-1 \\
1\end{array}\right]$} & {$\left[\begin{array}{r}0 \\
-1\end{array}\right]$} & {$\left[\begin{array}{l}1 \\
0\end{array}\right]$} \\
\hline 5 & {$\left[\begin{array}{rr}0 & -1 \\
1 & 0 \\
0 & 1 \\
1 & 0\end{array}\right]$} & {$\left[\begin{array}{l}0 \\
1\end{array}\right]$} & {$\left[\begin{array}{l}1 \\
0\end{array}\right]$} & {$\left[\begin{array}{r}0 \\
-1\end{array}\right]$} & {$\left[\begin{array}{r}-1 \\
0\end{array}\right]$} \\
\hline
\end{tabular}

- Its exchange matrix is $B_{t_{1}}=B^{1}=\mu_{k}\left(B^{0}\right)$.

- Its coefficient system is $\mathbf{y}_{t_{1}}=\left(y_{1}^{\prime}, \ldots, y_{n}^{\prime}\right)$, where the $y_{j}^{\prime}$ are given by $(2.3)$ evaluated in $\mathbb{P}$ :

$$
y_{j}^{\prime}= \begin{cases}y_{k}^{-1} & \text { if } j=k \\ y_{j} y_{k}^{\left[b_{k j}^{0}\right]_{+}} & \text {if } j \neq k .\end{cases}
$$

- Its cluster is $\mathbf{x}_{t_{1}}=\left(x_{1}^{\prime}, \ldots, x_{n}^{\prime}\right)$, where $x_{i}^{\prime}=x_{i}$ for $i \neq k$, and

$$
x_{k}^{\prime}=x_{k}^{-1}\left(y_{k} \prod x_{i}^{\left[b_{i k}\right]_{+}}+\prod x_{i}^{\left[-b_{i k}\right]_{+}}\right) .
$$

Finally, let $\left(\hat{y}_{1}, \ldots, \hat{y}_{n}\right)$ be given by $(6.2)$, and let $\left(\hat{y}_{1}^{\prime}, \ldots, \hat{y}_{n}^{\prime}\right)$ be obtained from them by $(2.3)$, where the addition is the one in the ambient field $\mathcal{F}$.

To prove (6.15), we evaluate the cluster variable $x_{\ell ; t} \in \mathcal{A}$ in two different ways by applying the formula (6.5) with respect to the seeds at $t_{0}$ and at $t_{1}$. Equating the two expressions yields the identity

$$
\frac{\left.F_{\ell ; t}^{B^{0} ; t_{0}}\right|_{\mathcal{F}}\left(\hat{y}_{1}, \ldots, \hat{y}_{n}\right)}{\left.F_{\ell ; t}^{B^{0} ; t_{0}}\right|_{\mathbb{P}}\left(y_{1}, \ldots, y_{n}\right)} \prod_{i} x_{i}^{g_{i}}=\frac{\left.F_{\ell ; t}^{B^{1} ; t_{1}}\right|_{\mathcal{F}}\left(\hat{y}_{1}^{\prime}, \ldots, \hat{y}_{n}^{\prime}\right)}{\left.F_{\ell ; t}^{B^{1} ; t_{1}}\right|_{\mathbb{P}}\left(y_{1}^{\prime}, \ldots, y_{n}^{\prime}\right)} \prod_{i}\left(x_{i}^{\prime}\right)^{g_{i}^{\prime}}
$$




\section{Cluster algebras IV}

Note that the denominator on the left of (6.19) is equal to 1 by (5.5). By the same token, it is easy to see that the denominator on the right of (6.19) is equal to $y_{k}^{h_{k}^{\prime}}$ (see (6.16)). Therefore (6.19) simplifies to

$$
\left.F_{\ell ; t}^{B^{0} ; t_{0}}\right|_{\mathcal{F}}\left(\hat{y}_{1}, \ldots, \hat{y}_{n}\right) \prod_{i} x_{i}^{g_{i}}=\left.F_{\ell ; t}^{B^{1} ; t_{1}}\right|_{\mathcal{F}}\left(\hat{y}_{1}^{\prime}, \ldots, \hat{y}_{n}^{\prime}\right) y_{k}^{-h_{k}^{\prime}} \prod_{i}\left(x_{i}^{\prime}\right)^{g_{i}^{\prime}}
$$

We now specialize (6.20) by setting $x_{i}=1$ for all $i$. By (6.18), $x_{k}^{\prime}$ specializes to $y_{k}+1$, while by (6.2), each $\hat{y}_{j}$ specializes to $y_{j}$. As for the elements $\hat{y}_{j}^{\prime}$, they specialize to the tuple of elements of $\mathcal{F}$ obtained from the $y_{j}$ by $(2.3)$, with the addition again understood as the one in $\mathcal{F}$. To distinguish the latter elements from those in (6.17), we denote the specialized $\hat{y}_{j}^{\prime}$ by $\bar{y}_{j}^{\prime}$; we can thus assume that the elements $\bar{y}_{j}^{\prime}$ belong to the universal semifield $\mathbb{Q}_{\mathrm{sf}}\left(y_{1}, \ldots, y_{n}\right)$ and are given by $(2.3)$ there. In this notation, (6.20) specializes to

$$
F_{\ell ; t}^{B^{0} ; t_{0}}\left(y_{1}, \ldots, y_{n}\right)=F_{\ell ; t}^{B^{1} ; t_{1}}\left(\bar{y}_{1}^{\prime}, \ldots, \bar{y}_{n}^{\prime}\right) y_{k}^{-h_{k}^{\prime}}\left(y_{k}+1\right)^{g_{k}^{\prime}}
$$

Using the universality of $\mathbb{Q}_{\mathrm{sf}}\left(y_{1}, \ldots, y_{n}\right)$, we conclude that (6.21) implies the following:

$$
\left.F_{\ell ; t}^{B^{0} ; t_{0}}\right|_{\mathcal{F}}\left(\hat{y}_{1}, \ldots, \hat{y}_{n}\right)=\left.F_{\ell ; t}^{B^{1} ; t_{1}}\right|_{\mathcal{F}}\left(\hat{y}_{1}^{\prime}, \ldots, \hat{y}_{n}^{\prime}\right) \hat{y}_{k}^{-h_{k}^{\prime}}\left(\hat{y}_{k}+1\right)^{g_{k}^{\prime}} .
$$

Substituting (6.22) into (6.20) and performing cancellations, we obtain

$$
\prod_{i} x_{i}^{g_{i}}=\left(\hat{y}_{k} / y_{k}\right)^{h_{k}^{\prime}}\left(\hat{y}_{k}+1\right)^{-g_{k}^{\prime}} \prod_{i}\left(x_{i}^{\prime}\right)^{g_{i}^{\prime}}
$$

It follows from (6.2) and (6.18) that

$$
\hat{y}_{k} / y_{k}=\prod_{i} x_{i}^{b_{i k}^{0}}, \quad \hat{y}_{k}+1=\prod_{i} x_{i}^{-\left[-b_{i k}^{0}\right]+} x_{k} x_{k}^{\prime} .
$$

Substituting these expressions into (6.23), we see that the powers of $x_{k}^{\prime}$ on the right cancel out, so both sides are monomials in $x_{1}, \ldots, x_{n}$. The desired formula (6.15) is obtained by equating the exponents of each $x_{i}$ on both sides.

As a byproduct of the above proof, we can express the $\mathbf{g}$-vector $\mathbf{g}_{\ell ; t}^{B^{0} ; t_{0}}$ in terms of $F$-polynomials.

Proposition 6.9. In the notation of Proposition 6.8, the component $g_{k}$ of the $\mathbf{g}$-vector $\mathbf{g}_{\ell ; t}^{B^{0} ; t_{0}}$ is given by

$$
u^{g_{k}}=\frac{\left.F_{\ell, t}^{B^{0} ; t_{0}}\right|_{\operatorname{Trop}(u)}\left(u^{\left[-b_{k 1}^{0}\right]_{+}}, \ldots, u^{-1}, \ldots, u^{\left[-b_{k n}^{0}\right]_{+}}\right)}{\left.F_{\ell, t}^{B^{1} ; t_{1}}\right|_{\operatorname{Trop}(u)}\left(u^{\left[b_{k 1}^{0}\right]_{+}}, \ldots, u^{-1}, \ldots, u^{\left[b_{k n}^{0}\right]_{+}}\right)}
$$

( $u^{-1}$ in the $k$ th position).

Proof. Define the integer $h_{k}$ by

$$
u^{h_{k}}=\left.F_{\ell, t}^{B^{0} ; t_{0}}\right|_{\operatorname{Trop}(u)}\left(u^{\left[-b_{k 1}^{0}\right]_{+}}, \ldots, u^{-1}, \ldots, u^{\left[-b_{k n}^{0}\right]_{+}}\right) \quad\left(u^{-1} \text { in the } k \text { th position }\right) .
$$

Then the desired equality (6.24) takes the form

$$
g_{k}=h_{k}-h_{k}^{\prime}
$$

To prove (6.26), we exploit the symmetry between $t_{0}$ and $t_{1}$. To do this, we use the formula obtained from (6.21) by interchanging $t_{0}$ and $t_{1}$. Comparing (6.25) with (6.16) (and using the first case in the matrix mutation rule $(2.2)$ ), we see that interchanging $t_{0}$ and $t_{1}$ replaces $h_{k}^{\prime}$ with $h_{k}$, and $g_{k}^{\prime}$ with $g_{k}$; thus, the resulting counterpart of (6.21) takes the form

$$
F_{\ell ; t}^{B^{1} ; t_{1}}\left(\bar{y}_{1}^{\prime}, \ldots, \bar{y}_{n}^{\prime}\right)=F_{\ell ; t}^{B^{0} ; t_{0}}\left(y_{1}, \ldots, y_{n}\right)\left(\bar{y}_{k}^{\prime}\right)^{-h_{k}}\left(\bar{y}_{k}^{\prime}+1\right)^{g_{k}} .
$$




\section{S. Fomin And A. Zelevinsky}

Using the fact that $\bar{y}_{k}^{\prime}=y_{k}^{-1}$ (by the first case in (2.3)), we can rewrite (6.27) as follows:

$$
F_{\ell ; t}^{B^{0} ; t_{0}}\left(y_{1}, \ldots, y_{n}\right)=F_{\ell ; t}^{B^{1} ; t_{1}}\left(\bar{y}_{1}^{\prime}, \ldots, \bar{y}_{n}^{\prime}\right) y_{k}^{g_{k}-h_{k}}\left(y_{k}+1\right)^{-g_{k}} .
$$

Comparing (6.28) with (6.21) yields the desired equality (6.26).

We conclude this section with several conjectural properties of the g-vectors. The first sharpens (6.26).

Conjecture 6.10. In the notation of Proposition 6.8 and (6.25), we have

$$
h_{k}^{\prime}=-\left[g_{k}\right]_{+}, \quad h_{k}=-\left[-g_{k}\right]_{+}=\min \left(0, g_{k}\right) \text {. }
$$

The next conjecture somewhat surprisingly expresses the $\mathbf{g}$-vector $\mathbf{g}_{\ell ; t}^{B^{0} ; t_{0}}$ in terms of the polynomial $F_{\ell ; t}^{B^{0} ; t_{0}}$ alone.

Conjecture 6.11. Suppose that $B^{0}, t_{0}, \ell$ and $t$ are such that the polynomial $F_{\ell ; t}^{B^{0} ; t_{0}}$ is not identically equal to 1 . Then the $\mathbf{g}$-vector $\mathbf{g}_{\ell ; t}^{B^{0} ; t_{0}}=\left[\begin{array}{c}g_{1} \\ \vdots \\ g_{n}\end{array}\right]$ is given by

$$
u_{1}^{g_{1}} \cdots u_{n}^{g_{n}}=\frac{\left.F_{\ell, t}^{B^{0} ; t_{0}}\right|_{\operatorname{Trop}\left(u_{1}, \ldots, u_{n}\right)}\left(u_{1}^{-1}, \ldots, u_{n}^{-1}\right)}{\left.F_{\ell, t}^{B^{0} ; t_{0}}\right|_{\operatorname{Trop}\left(u_{1}, \ldots, u_{n}\right)}\left(\prod_{i} u_{i}^{b_{i 1}^{0}}, \ldots, \prod_{i} u_{i}^{b_{i n}^{0}}\right)} .
$$

To state our last conjecture, the following terminology comes in handy.

Definition 6.12. We say that a collection of vectors in $\mathbb{Z}^{n}$ (or $\mathbb{R}^{n}$ ) are sign-coherent (to each other) if, for any $i \in[1, n]$, the $i$ th coordinates of all of these vectors are either all non-negative or all non-positive.

Conjecture 6.13. For any given $B^{0}, t_{0}$, and $t$ as above, the vectors $\mathbf{g}_{1 ; t}^{B^{0} ; t_{0}}, \ldots, \mathbf{g}_{n ; t}^{B^{0} ; t_{0}}$ are sign-coherent.

The significance of these conjectures will become clearer in the following sections.

\section{Cluster monomials and their parameterizations}

Definition 7.1 [FZ04, Definition 4.15]. A cluster monomial in a cluster algebra $\mathcal{A}$ is a monomial in cluster variables all of which belong to the same cluster. Thus, a cluster monomial at a vertex $t \in \mathbb{T}_{n}$ is a product of the form

$$
x_{\mathbf{a} ; t}=\prod_{\ell} x_{\ell ; t}^{a_{\ell}} \in \mathcal{F} \quad \text { with } \mathbf{a}=\left[\begin{array}{c}
a_{1} \\
\vdots \\
a_{n}
\end{array}\right] \in \mathbb{Z}_{\geqslant 0}^{n} .
$$

Cluster monomials are destined to play an important role in the emerging structural theory of cluster algebras and its applications. We refer the reader to [FZ04, § 4.3] for a discussion of the properties of cluster monomials, both proven and conjectural. Based on the results for rank 2 obtained in [SZ04], we expect every cluster monomial to belong to the 'canonical basis' that has yet to be defined for an arbitrary cluster algebra. Even so, the following basic property has not been established in general.

Conjecture 7.2 [FZ04, Conjecture 4.16]. Cluster monomials in any cluster algebra are linearly independent over the ground ring. 


\section{Cluster algebras IV}

For cluster algebras of finite type, this conjecture is proved in Theorem 11.2.

In this section, we discuss two families of combinatorial parameterizations of cluster monomials by integer vectors in $\mathbb{Z}^{n}$. (They are somewhat reminiscent of Lusztig's and string parameterizations of canonical bases in the theory of quantum groups.) One of these parameterizations (by denominator vectors) has already appeared in [FZ03a, FZ04]; another (by g-vectors) is new. (Note that in $\S 6$, g-vectors were defined in restricted generality, for the case of principal coefficients only.)

We start by recalling the notion of denominator vectors (see, e.g., [FZ04, §4.3]). Let $\mathbf{x}=$ $\left(x_{1}, \ldots, x_{n}\right)$ be a labeled cluster from some seed in $\mathcal{A}$. By Theorem 3.5, every non-zero element $z \in \mathcal{A}$ can be uniquely written as

$$
z=\frac{N\left(x_{1}, \ldots, x_{n}\right)}{x_{1}^{d_{1}} \cdots x_{n}^{d_{n}}}
$$

where $N\left(x_{1}, \ldots, x_{n}\right)$ is a polynomial with coefficients in $\mathbb{Z} \mathbb{P}$ which is not divisible by any cluster variable $x_{i} \in \mathbf{x}$. We denote

$$
\mathbf{d}(z)=\mathbf{d}_{\mathbf{x}}(z)=\left[\begin{array}{c}
d_{1} \\
\vdots \\
d_{n}
\end{array}\right] \in \mathbb{Z}^{n}
$$

and call the integer vector $\mathbf{d}(z)$ the denominator vector of $z$ with respect to the cluster $\mathbf{x}$. Note that the map $z \mapsto \mathbf{d}(z)$ has the following multiplicative property:

$$
\mathbf{d}\left(z_{1} z_{2}\right)=\mathbf{d}\left(z_{1}\right)+\mathbf{d}\left(z_{2}\right)
$$

Suppose that $\mathcal{A}$ is associated with a (labeled) cluster pattern $t \mapsto\left(\mathbf{x}_{t}, \mathbf{y}_{t}, B_{t}\right)$ on $\mathbb{T}_{n}$, and that $\mathbf{x}$ is the cluster of the initial seed (3.1) at $t_{0}$. We use the notation

$$
\mathbf{d}_{\ell ; t}^{B^{0} ; t_{0}}=\mathbf{d}_{\mathbf{x}}\left(x_{\ell ; t}\right)
$$

for the denominator vectors of cluster variables. This notation is unambiguous since it is easy to see that $\mathbf{d}_{\mathbf{x}}\left(x_{\ell ; t}\right)$ is independent of the choice of the coefficient system $\mathbf{y}=\mathbf{y}_{t_{0}}$; in fact, the mutation mechanism for generating cluster variables makes it clear that the whole Newton polytope of $x_{\ell ; t}$ (as a Laurent polynomial in $x_{1}, \ldots, x_{n}$ ) is independent of this choice.

More explicitly, the vectors $\mathbf{d}_{\ell ; t}=\mathbf{d}_{\ell ; t}^{B^{0} ; t_{0}}$ are uniquely determined by the initial conditions

$$
\mathbf{d}_{\ell ; t_{0}}^{B^{0} ; t_{0}}=-\mathbf{e}_{\ell}
$$

(as before, $\mathbf{e}_{1}, \ldots, \mathbf{e}_{n}$ are the standard basis vectors in $\mathbb{Z}^{n}$ ) together with the recurrence relations implied by the exchange relation (2.4):

$$
\mathbf{d}_{\ell ; t^{\prime}}= \begin{cases}\mathbf{d}_{\ell ; t} & \text { if } \ell \neq k \\ -\mathbf{d}_{k ; t}+\max \left(\sum_{i}\left[b_{i k}^{t}\right]_{+} \mathbf{d}_{i ; t}, \sum_{i}\left[-b_{i k}^{t}\right]_{+} \mathbf{d}_{i ; t}\right) & \text { if } \ell=k\end{cases}
$$

for $t \stackrel{k}{L} t^{\prime}$; here and in what follows, the operations max, min, $[a]_{+}$, etc., on vectors are performed component-wise.

Example 7.3. The denominator vectors $\mathbf{d}_{\ell ; t}^{B^{0} ; t_{0}}$ for a cluster algebra of type $A_{2}$ (cf. Examples 2.10, 3.4 , and 6.7) are shown in the fourth column of Table 4.

As above, let $\mathcal{A}$ be a cluster algebra with the initial exchange matrix $B_{t_{0}}=B^{0}$. If a cluster variable $x_{\ell ; t}$ belongs to the initial cluster, that is, $x_{\ell ; t}=x_{i ; t_{0}}$ for some $i$, then its denominator is given by (7.6). Otherwise, we expect its denominator vector to have the following properties. 


\section{S. Fomin And A. Zelevinsky}

ConjeCture 7.4. Suppose that $x_{\ell ; t}$ does not belong to the initial cluster. Let $\mathbf{d}_{\ell ; t}^{B^{0} ; t_{0}}=\left[\begin{array}{c}d_{1} \\ \vdots \\ d_{n}\end{array}\right]$ be
the denominator vector of $x_{\ell ; t}$. Then:

(1) all components $d_{i}$ are non-negative;

(2) we have $d_{i}=0$ if and only if there is a cluster containing both $x_{\ell ; t}$ and $x_{i ; t_{0}}$;

(3) each component $d_{i}$ depends only on $x_{\ell ; t}$ and $x_{i ; t_{0}}$, not on the vertices $t_{0}$ and $t$.

We note that Conjecture 7.4 implies the following counterpart of Conjecture 6.13 (cf. Definition 6.12).

Conjecture 7.5. For any $t$ and $t_{0}$, the vectors $\mathbf{d}_{1 ; t}^{B^{0} ; t_{0}}, \ldots, \mathbf{d}_{n ; t}^{B^{0} ; t_{0}}$ are sign-coherent.

Using (7.4), we can extend the notation (7.5) from cluster variables to cluster monomials (cf. (7.1)) by setting

$$
\mathbf{d}_{\mathbf{a} ; t}^{B^{0} ; t_{0}}=\mathbf{d}_{\mathbf{x}}\left(x_{\mathbf{a} ; t}\right)=\sum_{\ell=1}^{n} a_{\ell} \mathbf{d}_{\ell ; t}^{B^{0} ; t_{0}} .
$$

In particular, in view of (7.6), we have

$$
\mathbf{d}_{\mathbf{a} ; t_{0}}^{B^{0} ; t_{0}}=-\mathbf{a} \quad\left(\mathbf{a} \in \mathbb{Z}_{\geqslant 0}^{n}\right) .
$$

Conjecture 7.6 [FZ04, Conjecture 4.17]. Different cluster monomials have different denominators with respect to a given initial seed $\Sigma_{t_{0}}$. In particular, for any fixed $t \in \mathbb{T}_{n}$, the correspondence $\mathbf{a} \mapsto \mathbf{d}_{\mathbf{a} ; t}^{B^{0} ; t_{0}}$ is an embedding $\mathbb{Z}_{\geqslant 0}^{n} \rightarrow \mathbb{Z}^{n}$, hence the vectors $\mathbf{d}_{1 ; t}^{B^{0} ; t_{0}}, \ldots, \mathbf{d}_{n ; t}^{B^{0} ; t_{0}}$ form a basis of the vector space $\mathbb{Q}^{n}$.

Remark 7.7. If the initial seed $\Sigma_{t_{0}}$ is acyclic (see [BFZ05]), we expect the vectors $\mathbf{d}_{1 ; t}^{B^{0} ; t_{0}}, \ldots, \mathbf{d}_{n ; t}^{B^{0} ; t_{0}}$ to form a $\mathbb{Z}$-basis of the lattice $\mathbb{Z}^{n}$. We note that this property does not hold in general. The simplest counterexample is in finite type $A_{3}$, for a non-acyclic seed with the exchange matrix

$$
B^{0}=B_{t_{0}}=\left[\begin{array}{rrr}
0 & 1 & -1 \\
-1 & 0 & 1 \\
1 & -1 & 0
\end{array}\right]
$$

Let $t$ be the vertex connected to $t_{0}$ by the path

$$
t_{0} \stackrel{1}{-} t_{1} \stackrel{2}{-} t_{2} \stackrel{3}{-} t_{3} \stackrel{1}{-} t .
$$

Applying the recurrence (7.7), we compute the vectors $\mathbf{d}_{\ell ; t}^{B^{0} ; t_{0}}$ :

$$
\mathbf{d}_{1 ; t}^{B^{0} ; t_{0}}=\left[\begin{array}{l}
0 \\
1 \\
1
\end{array}\right], \quad \mathbf{d}_{2 ; t}^{B^{0} ; t_{0}}=\left[\begin{array}{l}
1 \\
1 \\
0
\end{array}\right], \quad \mathbf{d}_{3 ; t^{0} ; t_{0}}=\left[\begin{array}{l}
1 \\
0 \\
1
\end{array}\right] .
$$

These vectors generate a proper sublattice of index 2 in $\mathbb{Z}^{3}$.

In view of Remark 7.7, it is unlikely that there exists a simple formula relating the denominator vectors $\mathbf{d}_{\ell ; t}^{B^{0} ; t_{0}}$ and $\mathbf{d}_{\ell ; t}^{B^{1} ; t_{1}}$ (for $t_{0} \stackrel{k}{-} t_{1}$ and $\mu_{k}\left(B^{0}\right)=B^{1}$ ). This feature is expected to be rectified by passing from the denominator vectors to the $\mathbf{g}$-vector parameterization. Introducing the latter will require a little preparation.

Let $\mathcal{A}$ be a cluster algebra of geometric type associated to an $m \times n$ matrix $\tilde{B}_{t_{0}}=\tilde{B}^{0}$ (see $(2.13)$ and (2.14)). We assume that $\mathcal{A}$ satisfies the following property:

$$
\text { The matrix } \tilde{B}^{0} \text { has full rank } n \text {. }
$$




\section{Cluster algebras IV}

As shown in [GSV03, Lemma 1.2] and [BFZ05, Lemma 3.2], the same is then true for all matrices $\tilde{B}_{t}$. For instance, all of the algebras with principal coefficients satisfy (7.10). The property (7.10) easily implies that, for every $t$, the elements

$$
\hat{y}_{j ; t}=y_{j ; t} \prod_{i=1}^{n} x_{i ; t}^{b_{i j}^{t}}=\prod_{i=1}^{m} x_{i ; t}^{b_{i j}^{t}} \quad(j \in[1, n])
$$

(cf. (3.7)) are algebraically independent in $\mathcal{F}$; here the elements $x_{i ; t}=x_{i}$ for $n<i \leqslant m$ are the generators of the coefficient semifield $\mathbb{P}=\operatorname{Trop}\left(x_{n+1}, \ldots, x_{m}\right)$.

Let $\mathcal{M}$ denote the set of all non-zero elements $z \in \mathcal{A}$ that can be written in the form

$$
z=R\left(\hat{y}_{1 ; t}, \ldots, \hat{y}_{n ; t}\right) \prod_{i=1}^{m} x_{i ; t}^{a_{i}}
$$

for some vertex $t \in \mathbb{T}_{n}$, where $R$ is a rational function in $n$ variables with coefficients in $\mathbb{Q}$, and $a_{i} \in \mathbb{Z}$ for $i \in[1, m]$. In view of the formulas (3.8) and (3.11), if $z$ is of the form (7.12) for some $t$, then it can be written in the same form for any choice of $t$. Therefore (or by Corollary 6.3), each cluster variable - hence each cluster monomial - belongs to $\mathcal{M}$. Clearly, $\mathcal{M}$ is a subgroup of the multiplicative group of the ambient field $\mathcal{F}$.

We call a rational function $R\left(u_{1}, \ldots, u_{n}\right) \in \mathbb{Q}\left(u_{1}, \ldots, u_{n}\right)$ primitive if it can be written as a ratio of two polynomials not divisible by any $u_{i}$. In view of (7.11), any $z \in \mathcal{M}$ can be written in the form (7.12) with $R$ primitive. We claim that such a presentation is unique.

Proposition 7.8. If $\mathcal{A}$ satisfies (7.10), then every $z \in \mathcal{M}$ has a unique presentation in the form (7.12) with $R$ primitive.

Proof. It is enough to show that if

$$
P\left(\hat{y}_{1 ; t}, \ldots, \hat{y}_{n ; t}\right)=Q\left(\hat{y}_{1 ; t}, \ldots, \hat{y}_{n ; t}\right) \prod_{i=1}^{m} x_{i ; t}^{a_{i}}
$$

for some primitive polynomials $P$ and $Q$ and some integers $a_{1}, \ldots, a_{m}$, then $a_{i}=0$ for all $i$, and so $P=Q$. Since each $\hat{y}_{j ; t}$ is a Laurent monomial in $m$ independent variables $x_{1 ; t}, \ldots, x_{m ; t}$, the relation (7.13) implies that $\prod_{i=1}^{m} x_{i ; t}^{a_{i}}$ can be expressed as a Laurent monomial in $\hat{y}_{1 ; t}, \ldots, \hat{y}_{n ; t}$. Since $P$ and $Q$ are primitive, their ratio can be a Laurent monomial only if they are equal to each other, and we are done.

Now everything is in place for defining the $\mathbf{g}$-vector parameterizations (of $\mathcal{M}$ ).

Definition 7.9. For any $z \in \mathcal{M}$ and any $t \in \mathbb{T}_{n}$, the g-vector of $z$ with respect to $t$ is the vector $\mathbf{g}_{t}(z) \in \mathbb{Z}^{n}$ defined as follows: if $z$ is expressed in the form (7.12) with $R$ primitive, then we set $\mathbf{g}_{t}(z)=\left[\begin{array}{c}a_{1} \\ \vdots \\ a_{n}\end{array}\right]$. (This is well defined by Proposition 7.8.)

Definition 7.9 implies at once the following analogue of (7.4):

$$
\mathbf{g}_{t}\left(z_{1} z_{2}\right)=\mathbf{g}_{t}\left(z_{1}\right)+\mathbf{g}_{t}\left(z_{2}\right) .
$$

By Corollary 6.3 and Proposition 5.2, we have

$$
\mathbf{g}_{t_{0}}\left(x_{\ell ; t}\right)=\mathbf{g}_{\ell ; t}^{B^{0} ; t_{0}}
$$

as defined by (6.4); so our terminology is consistent with that of $\S 6$. 


\section{S. Fomin And A. Zelevinsky}

In analogy with (7.8), we use (7.14) to extend the notation (7.15) from cluster variables to cluster monomials by setting

$$
\mathbf{g}_{\mathbf{a} ; t}^{B^{0} ; t_{0}}=\mathbf{g}_{t_{0}}\left(x_{\mathbf{a} ; t}\right)=\sum_{\ell=1}^{n} a_{\ell} \mathbf{g}_{\ell ; t}^{B^{0} ; t_{0}}
$$

In particular, we have (cf. (7.9))

$$
\mathbf{g}_{\mathbf{a} ; t_{0}}^{B^{0} t_{0}}=\mathbf{a} \quad\left(\mathbf{a} \in \mathbb{Z}_{\geqslant 0}^{n}\right) .
$$

We suggest the following counterpart of Conjecture 7.6.

\section{CONJECTURE 7.10.}

(1) Different cluster monomials have different $\mathbf{g}$-vectors with respect to a given initial seed.

(2) For every $t \in \mathbb{T}_{n}$, the vectors $\mathbf{g}_{1 ; t}^{B^{0} ; t_{0}}, \ldots, \mathbf{g}_{n ; t}^{B^{0} ; t_{0}}$ form a $\mathbb{Z}$-basis of the lattice $\mathbb{Z}^{n}$.

Remark 7.11. It is not hard to see that part (1) of Conjecture 7.10 together with Conjecture 5.4 imply Conjecture 7.2 (in a cluster algebra satisfying (7.10)). Indeed, assuming Conjecture 5.4 and applying Corollary 6.3, we see that the Laurent expansion of any cluster monomial $z$ in terms of the elements $x_{i}=x_{i ; t_{0}}(i \in[1, m])$ contains a Laurent monomial $x_{1}^{a_{1}} \cdots x_{m}^{a_{m}}$ such that $\mathbf{a}=\left[\begin{array}{c}a_{1} \\ \vdots \\ a_{n}\end{array}\right]=\mathbf{g}_{t_{0}}(z)$, and the exponent vector of any other Laurent monomial in the same expansion is obtained from a by adding some non-negative integer linear combination of the columns of $\tilde{B}^{0}$. Since the columns of $\tilde{B}^{0}$ are assumed to be linearly independent, the monomial $x_{1}^{a_{1}} \cdots x_{m}^{a_{m}}$ is the leading term of $z$ with respect to an appropriate term order. Then part (1) of Conjecture 7.10 implies that different cluster monomials have different leading terms, and so are linearly independent.

The property in part (2) of Conjecture 7.10 is stronger than the corresponding (conjectural) property of the denominator vectors (cf. Remark 7.7). Another (conjectural) advantage of the $\mathrm{g}$-vectors is the following nice transition rule.

Conjecture 7.12. Let $t_{0} \stackrel{k}{-} t_{1}$ be two adjacent vertices in $\mathbb{T}_{n}$, and let $B^{1}=\mu_{k}\left(B^{0}\right)$. Then, for any $t \in \mathbb{T}_{n}$ and $\mathbf{a} \in \mathbb{Z}_{\geqslant 0}^{n}$, the $\mathbf{g}$-vectors $\mathbf{g}_{\mathbf{a} ; t}^{B^{0} ; t_{0}}=\left(g_{1}, \ldots, g_{n}\right)$ and $\mathbf{g}_{\mathbf{a} ; t}^{B^{1} ; t_{1}}=\left(g_{1}^{\prime}, \ldots, g_{n}^{\prime}\right)$ are related as follows:

$$
g_{j}^{\prime}= \begin{cases}-g_{k} & \text { if } j=k ; \\ g_{j}+\left[b_{j k}^{0}\right]_{+} g_{k}-b_{j k}^{0} \min \left(g_{k}, 0\right) & \text { if } j \neq k .\end{cases}
$$

Remark 7.13. Conjecture 7.12 is easily seen to be a consequence of Conjectures 6.10 and 6.13 . Indeed, combining (6.15) with (6.29) (and interchanging $t_{0}$ and $t_{1}$ ), we conclude that (7.18) holds for the $\mathbf{g}$-vectors of cluster variables; the fact that (7.18) extends to cluster monomials follows from Conjecture 6.13.

Remark 7.14. If $\mathbf{g}_{1}, \ldots, \mathbf{g}_{n}$ are sign-coherent vectors forming a $\mathbb{Z}$-basis in $\mathbb{Z}^{n}$, then the transformation (7.18) sends them to a $\mathbb{Z}$-basis in $\mathbb{Z}^{n}$. Thus, the validity of part (2) of Conjecture 7.10 for all initial seeds would follow from its validity for any single choice of $t_{0}$ in conjunction with Conjectures 6.13 and 7.12 .

Remark 7.15. Let $\mathbb{Z}_{\text {min,+ }}$ denote the set of integers supplied with the following semifield structure: the multiplication in $\mathbb{Z}_{\text {min, }+}$ is the usual addition of integers, while the 'addition' is given by $a \oplus b=$ $\min (a, b)$. Comparing (7.18) with (2.3), we see that Conjecture 7.12 can be restated as follows: 


\section{Cluster algebras IV}

for every cluster monomial $z$, the correspondence $t \mapsto\left(\mathrm{g}_{t}(z), B_{t}^{\mathrm{T}}\right)$ (here $B_{t}^{\mathrm{T}}$ denotes the transpose of $B_{t}$ ) is a $Y$-pattern (see Definition 2.9) with values in $\mathbb{Z}_{\min ,+}$. The fact that the exchange matrices $B_{t}$ get replaced by their transposes brings to mind the Langlands duality. According to part (1) of Conjecture 7.10, each cluster monomial gives rise to a certain 'Langlands dual tropical $Y$-pattern'. This conjecture is consistent with the series of conjectures by Fock and Goncharov in [FG03, §5] on a 'canonical' basis in a cluster algebra. In fact, our construction of g-vectors was motivated by a desire to understand [FG03, Conjecture 5.1] and make the parameterizations introduced there more explicit.

We conclude this section by a proposition and a conjecture relating denominator vectors of cluster variables to the corresponding $\mathbf{g}$-vectors.

Proposition 7.16. Assume that condition (7.10) holds. Then the denominator vector $\mathbf{d}_{\ell ; t}^{B^{0} ; t_{0}}=$ $\left[\begin{array}{c}d_{1} \\ \vdots \\ d_{n}\end{array}\right]$ and the $\mathbf{g}$-vector $\mathbf{g}_{\ell ; t}^{B^{0} ; t_{0}}=\left[\begin{array}{c}g_{1} \\ \vdots \\ g_{n}\end{array}\right]$ of the same cluster variable with respect to the same initial seed satisfy the following relation:

$$
u_{1}^{-d_{1}-g_{1}} \cdots u_{n}^{-d_{n}-g_{n}}=\left.F_{\ell, t}^{B^{0} ; t_{0}}\right|_{\operatorname{Trop}\left(u_{1}, \ldots, u_{n}\right)}\left(\prod_{i} u_{i}^{b_{i 1}^{0}}, \ldots, \prod_{i} u_{i}^{b_{i n}^{0}}\right) .
$$

Proof. Remembering the definition (7.2) of the denominator vector, we see that (7.19) is a consequence of (6.5).

In view of Proposition 7.16, Conjecture 6.11 can be equivalently restated as follows.

Conjecture 7.17. Suppose that $B^{0}, t_{0}, \ell$ and $t$ are such that the cluster variable $x_{\ell ; t}$ does not belong to the cluster at $t_{0}$. Then the denominator vector $\mathbf{d}_{\ell ; t}^{B^{0} ; t_{0}}=\left[\begin{array}{c}d_{1} \\ \vdots \\ d_{n}\end{array}\right]$ is given by

$$
u_{1}^{-d_{1}} \cdots u_{n}^{-d_{n}}=\left.F_{\ell, t}^{B^{0} ; t_{0}}\right|_{\operatorname{Trop}\left(u_{1}, \ldots, u_{n}\right)}\left(u_{1}^{-1}, \ldots, u_{n}^{-1}\right) .
$$

Remark 7.18. Comparing Conjecture 7.17 with Conjecture 5.5, we see that their conjunction can be stated as follows: the vector $\left[\mathbf{d}_{\ell ; t}^{B^{0} ; t_{0}}\right]_{+}$appears as the vector of exponents in the unique monomial in the polynomial $F_{\ell ; t}^{B^{0} ; t_{0}}\left(y_{1}, \ldots, y_{n}\right)$ that is divisible by all other occurring monomials.

\section{Bipartite belt and $Y$-systems}

Definition 8.1. We call a (labeled) seed $(\mathbf{x}, \mathbf{y}, B)$ (and its exchange matrix $B=\left(b_{i j}\right)$ ) bipartite if there is a function $\varepsilon:[1, n] \rightarrow\{1,-1\}$ such that, for all $i$ and $j$,

$$
b_{i j}>0 \Longrightarrow\left\{\begin{array}{l}
\varepsilon(i)=1, \\
\varepsilon(j)=-1 .
\end{array}\right.
$$

For the rest of this paper, we are working in a cluster algebra $\mathcal{A}=\mathcal{A}\left(\mathbf{x}_{0}, \mathbf{y}_{0}, B\right)$ of rank $n$ with a bipartite initial exchange matrix $B=\left(b_{i j}\right)$.

The Cartan counterpart of $B$ (see [FZ03a, (1.6)]) is the (generalized) Cartan matrix $A=A(B)=$ $\left(a_{i j}\right)$ defined by

$$
a_{i j}= \begin{cases}2 & \text { if } i=j \\ -\left|b_{i j}\right| & \text { if } i \neq j\end{cases}
$$




\section{S. Fomin And A. Zelevinsky}

The Coxeter graph of $A$ has the vertices $1, \ldots, n$, with $i$ and $j$ joined by an edge whenever $a_{i j}<0$. A Cartan matrix $A$ is the Cartan counterpart of a bipartite matrix $B$ if and only if the Coxeter graph of $A$ is bipartite, which explains the terminology.

Recall that $\mu_{k}$ denotes the mutation in direction $k$; we use the same notation for seeds, $Y$-seeds, and exchange matrices. Note that $\mu_{k} \mu_{\ell}=\mu_{\ell} \mu_{k}$ whenever $b_{k \ell}=0$. Therefore, the following two 'composite' mutations are well defined on bipartite seeds:

$$
\mu_{+}=\prod_{\varepsilon(k)=1} \mu_{k}, \quad \mu_{-}=\prod_{\varepsilon(k)=-1} \mu_{k} .
$$

Furthermore, each of $\mu_{+}$and $\mu_{-}$is involutive and transforms bipartite seeds to bipartite seeds; in fact, $\mu_{ \pm}(B)=-B$. In the terminology of [FZ03a, $\S 9.1$, these mutation transformations are 'shape-preserving'.

Definition 8.2 (Bipartite belt). The initial bipartite seed $\Sigma_{0}=\left(\mathbf{x}_{0}, \mathbf{y}_{0}, B\right)$ is naturally included into a bipartite belt consisting of the seeds

$$
\Sigma_{m}=\left(\mathbf{x}_{m}, \mathbf{y}_{m},(-1)^{m} B\right) \quad(m \in \mathbb{Z})
$$

defined by setting, for each $r>0$,

$$
\begin{gathered}
\Sigma_{r}=\left(\mathbf{x}_{r}, \mathbf{y}_{r},(-1)^{r} B\right)=\underbrace{\mu_{ \pm} \cdots \mu_{-} \mu_{+} \mu_{-}}_{r \text { factors }}\left(\Sigma_{0}\right), \\
\Sigma_{-r}=\left(\mathbf{x}_{-r}, \mathbf{y}_{-r},(-1)^{r} B\right)=\underbrace{\mu_{\mp} \cdots \mu_{+} \mu_{-} \mu_{+}}_{r \text { factors }}\left(\Sigma_{0}\right) .
\end{gathered}
$$

We write the cluster $\mathbf{x}_{m}$ and the coefficient tuple $\mathbf{y}_{m}$ as

$$
\mathbf{x}_{m}=\left(x_{1 ; m}, \ldots, x_{n ; m}\right), \quad \mathbf{y}_{m}=\left(y_{1 ; m}, \ldots, y_{n ; m}\right) .
$$

The mutation rules of Definition 2.4 imply that, for $i, j \in[1, n]$ and $m \in \mathbb{Z}$,

$$
\begin{gathered}
x_{i ; m+1}=x_{i ; m} \quad \text { if } \varepsilon(i)=(-1)^{m}, \\
y_{j ; m+1}=y_{j ; m}^{-1} \quad \text { if } \varepsilon(j)=(-1)^{m-1} .
\end{gathered}
$$

Hence, in studying the elements $x_{i ; m}$ and $y_{j ; m}$, we may concentrate on the families

$$
\begin{gathered}
\left\{x_{i ; m}: \varepsilon(i)=(-1)^{m}\right\}, \\
\left\{y_{j ; m}: \varepsilon(j)=(-1)^{m-1}\right\} .
\end{gathered}
$$

The following exchange relations are easy consequences of $(2.3)$ and $(2.4)$. First, for $\varepsilon(i)=(-1)^{m}$, we have

$$
y_{i ; m-1} y_{i ; m+1}=\prod_{\varepsilon(j)=-\varepsilon(i)}\left(y_{j ; m} \oplus 1\right)^{-a_{j i}} .
$$

Second, for $\varepsilon(j)=(-1)^{m-1}$, we have

$$
x_{j ; m-1} x_{j ; m+1}=\frac{y_{j ; m} \prod_{\varepsilon(i)=-\varepsilon(j)} x_{i ; m}^{-a_{i j}}+1}{y_{j ; m} \oplus 1} .
$$

Example 8.3 (Type $A_{2}$ ). Let

$$
B=\left[\begin{array}{rr}
0 & 1 \\
-1 & 0
\end{array}\right], \quad A=A(B)=\left[\begin{array}{rr}
2 & -1 \\
-1 & 2
\end{array}\right], \quad \varepsilon(1)=1, \quad \varepsilon(2)=-1 .
$$




\section{Cluster algebras IV}

TABLE 5. Bipartite dynamics in type $A_{2}$.

\begin{tabular}{r|cc|cc|cc}
\hline$m$ & $y_{1 ; m}$ & $y_{2 ; m}$ & $x_{1 ; m}$ & $x_{2 ; m}$ & $\alpha(1 ; m)$ & $\alpha(2 ; m)$ \\
\hline-1 & $u_{1}$ & & & $x_{2}$ & & $-\alpha_{2}$ \\
0 & & $u_{2}$ & $x_{1}$ & & \\
1 & $\frac{u_{2} \oplus 1}{u_{1}}$ & & & $\frac{x_{1} u_{2}+1}{x_{2}\left(u_{2} \oplus 1\right)}$ & \\
2 & & $\frac{u_{1} \oplus u_{2} \oplus 1}{u_{1} u_{2}}$ & $\frac{x_{1} u_{2}+x_{2} u_{1}+1}{\left(u_{1} \oplus u_{2} \oplus 1\right) x_{1} x_{2}}$ & & \\
3 & $\frac{u_{1} \oplus 1}{u_{2}}$ & & & $\frac{x_{2} u_{1}+1}{x_{1}\left(u_{1} \oplus 1\right)}$ & & \\
4 \\
4
\end{tabular}

(This choice of signs is consistent with [FZ03b, § 3.5].) Then $\mu_{+}=\mu_{1}, \mu_{-}=\mu_{2}$, and (8.4) and (8.5) become

$$
\begin{gathered}
\vdots \\
\left(\mathbf{x}_{-2}, \mathbf{y}_{-2}, B\right)=\mu_{2}\left(\mathbf{x}_{-1}, \mathbf{y}_{-1},-B\right) \\
\left(\mathbf{x}_{-1}, \mathbf{y}_{-1},-B\right)=\mu_{1}\left(\mathbf{x}_{0}, \mathbf{y}_{0}, B\right) \\
\left(\mathbf{x}_{1}, \mathbf{y}_{1},-B\right)=\mu_{2}\left(\mathbf{x}_{0}, \mathbf{y}_{0}, B\right) \\
\left(\mathbf{x}_{2}, \mathbf{y}_{2}, B\right)=\mu_{1}\left(\mathbf{x}_{1}, \mathbf{y}_{1},-B\right)
\end{gathered}
$$

in agreement with the notation used in Example 2.10. The families (8.9) and (8.10) take the form $\left\{x_{i ; m}: i \not \equiv m \bmod 2\right\}$ and $\left\{y_{j ; m}: j \equiv m \bmod 2\right\}$, respectively. These elements are shown in Table 5 , obtained by erasing half of the entries from Table 1 and substituting $y_{1}=u_{1}^{-1}$ and $y_{2}=u_{2}$, so that the $y_{j ; m}$ are expressed in terms of

$$
u_{1}=y_{1 ;-1}=y_{1}^{-1} \quad \text { and } \quad u_{2}=y_{2 ; 0}=y_{2}
$$

(cf. [FZ03b, Example 1.3]), whereas the $x_{i ; m}$ are expressed in terms of $u_{1}, u_{2}$,

$$
x_{1}=x_{1 ; 0} \quad \text { and } \quad x_{2}=x_{2 ;-1}=x_{2 ; 0} .
$$

Formula (8.11) can be viewed as a recurrence relation for the family (8.10) of elements $y_{j ; m}$. This recurrence is a natural generalization of Zamolodchikov's $Y$-systems [FZ03b, Zam91], so we refer to it as a (generalized) $Y$-system (with values in a semifield $\mathbb{P}$ ) associated with a (generalized) Cartan matrix $A$.

There are two natural choices for the initial data in a $Y$-system. The first choice utilized in [FZ03b] takes an arbitrary $n$-tuple $\left(u_{1}, \ldots, u_{n}\right) \in \mathbb{P}^{n}$ given by

$$
u_{i}= \begin{cases}y_{i ;-1} & \text { if } \varepsilon(i)=+1 \\ y_{i ; 0} & \text { if } \varepsilon(i)=-1 .\end{cases}
$$

An alternative choice is to use as the initial settings an arbitrary $n$-tuple $\mathbf{y}_{0}=\left(y_{1}, \ldots, y_{n}\right) \in \mathbb{P}^{n}$. 


\section{S. Fomin And A. Zelevinsky}

In view of (8.8), the two choices are related by

$$
u_{i}= \begin{cases}y_{i}^{-1} & \text { if } \varepsilon(i)=+1 \\ y_{i} & \text { if } \varepsilon(i)=-1\end{cases}
$$

so passing from one to another does not present any problems.

While dealing with $Y$-systems, we may (and will) work in the universal semifield $\mathbb{Q}_{\text {sf }}\left(y_{1}, \ldots, y_{n}\right)$ of Definition 2.1. Using $\mathbf{y}_{0}=\left(y_{1}, \ldots, y_{n}\right)$ as the initial data and remembering the notation introduced in Definition 3.10, we can write the solution of a $Y$-system as

$$
y_{j ; m}=Y_{j ; t_{m}}^{B ; t_{0}}\left(y_{1}, \ldots, y_{n}\right),
$$

where $t_{0} \in \mathbb{T}_{n}$ (respectively, $t_{m} \in \mathbb{T}_{n}$ ) is the vertex to which the labeled seed $\Sigma_{0}$ (respectively, $\Sigma_{m}$ ) is attached. (For a general semifield $\mathbb{P}$, one interprets (8.16) as the corresponding specialization of the rational functions $Y_{j ; t_{m}}^{B ; t_{0}}$.)

Remark 8.4. In a traditional $Y$-system [FZ03b, Zam91], $A$ is a Cartan matrix of finite type, the initial data have form $(8.14)$, and $\mathbb{P}=\mathbb{Q}_{\mathrm{sf}}\left(u_{1}, \ldots, u_{n}\right)$. Note that there is a notational difference between the $Y$-system relation (8.11) and its counterpart [FZ03b, (1.1)]: these two relations feature the exponents $-a_{j i}$ and $-a_{i j}$, respectively. Accordingly, while translating the results in [FZ03b] into our current notation (or vice versa), the Cartan matrix $A$ should be transposed.

Theorem 8.5 (Laurent phenomenon for generalized $Y$-systems). In the $Y$-system (8.11) associated with an arbitrary symmetrizable generalized Cartan matrix $A$, every element $y_{j ; m}$ with $\varepsilon(j)=$ $(-1)^{m-1}$ viewed as a rational function in the initial data $u_{1}, \ldots, u_{n}$ (or $\left.y_{1}, \ldots, y_{n}\right)$ is, in fact, an integer Laurent polynomial.

Theorem 8.5 generalizes the finite type result [FZ03b, Theorem 1.5]; note however that we do not obtain the positivity property established in [FZ03b].

Proof. The theorem follows from (8.16) and Proposition 3.15 once we realize that for $Y_{j ; t}=y_{j ; m}$ with $\varepsilon(j)=(-1)^{m-1}$, all of the exponents $b_{i j}$ in (3.18) are non-negative.

Example 8.6 ( $Y$-system of rank 2; cf. [FZ02, (6.9)]). For $n=2$, a (generalized) Cartan matrix is of the form

$$
A=\left[\begin{array}{rr}
2 & -b \\
-c & 2
\end{array}\right]
$$

where the integers $b$ and $c$ are either both positive or both equal to 0 . Setting $\varepsilon(1)=1$ and $\varepsilon(2)=-1$, and assuming that $\oplus$ is the ordinary addition, the $Y$-system becomes

$$
\begin{aligned}
& y_{2 ; m+1}=\frac{\left(y_{1 ; m}+1\right)^{b}}{y_{2 ; m-1}} \quad \text { if } m \text { is odd; } \\
& y_{1 ; m+1}=\frac{\left(y_{2 ; m}+1\right)^{c}}{y_{1 ; m-1}} \quad \text { if } m \text { is even. }
\end{aligned}
$$

Using the initial data $u_{1}=y_{1 ;-1}, u_{2}=y_{2 ; 0}(\mathrm{cf} .(8.14))$, we obtain

$y_{1 ; 1}=\frac{\left(u_{2}+1\right)^{c}}{u_{1}}, \quad y_{2 ; 2}=\frac{\left(\left(u_{2}+1\right)^{c} / u_{1}+1\right)^{b}}{u_{2}}, \quad y_{1 ; 3}=\left(\frac{\left(\left(u_{2}+1\right)^{c} / u_{1}+1\right)^{b}}{u_{2}}+1\right)^{c} \frac{u_{1}}{\left(u_{2}+1\right)^{c}}, \quad$ etc.

To illustrate Theorem 8.5: to see that $y_{1 ; 3}$ is indeed a Laurent polynomial in $u_{1}$ and $u_{2}$, we can rewrite it as

$$
y_{1 ; 3}=\frac{1}{u_{1}^{b c-1} u_{2}^{c}}\left(u_{1}^{b}+\frac{\left(\left(u_{2}+1\right)^{c}+u_{1}\right)^{b}-u_{1}^{b}}{u_{2}+1}\right)^{c} .
$$




\section{Cluster algebras IV}

Example $8.7\left(Y\right.$-system of type $\left.A_{2 r-1}^{(1)}\right)$. Let $n$ be an even integer at least 4 , and let $A$ be the Cartan matrix of affine type $A_{n-1}^{(1)}$. So the Coxeter graph of $A$ is a $n$-cycle, and $a_{i j}=-1$ for any two adjacent vertices $i$ and $j$. The corresponding $Y$-system can be described in simple terms as follows. Place initial variables $u_{1}, \ldots, u_{n}$ around a circle. Alternately color them red and blue. Replace each red variable $u$ by $(v+1)(w+1) / u$, where $v$ and $w$ are the two neighbors of $u$. Then do the same for all blue variables, then again for all the red ones, etc. Theorem 8.5 asserts that all rational functions obtained this way are integer Laurent polynomials in $u_{1}, \ldots, u_{n}$. In particular, if the initial values are all specialized to 1 , then all of the numbers generated in this $Y$-system become integers. We leave it to the reader to check that these integers are squares of Fibonacci numbers.

Without losing ground, we can assume that the matrix $A$ is indecomposable, that is, its Coxeter graph is connected. In most situations, the general case reduces to this one in an obvious way.

Zamolodchikov conjectured [Zam91] and we proved [FZ03b, Theorems 1.1 and 1.2] that if $A$ is an indecomposable Cartan matrix of finite type, then the corresponding $Y$-system exhibits the following periodicity property:

$$
y_{j ; m+2(h+2)}=y_{j ; m} \quad(m \in \mathbb{Z}, j \in[1, n]),
$$

where $h$ is the Coxeter number. (In the special case of type $A_{n}$, this was proved earlier in [FS95, GT96].) Our next result sharpens (8.17) as follows.

Theorem 8.8. Suppose that an exchange matrix $B$ is bipartite, and its Cartan counterpart $A=$ $A(B)$ is indecomposable.

(1) If $A$ is of finite type, then the corresponding bipartite belt (see Definition 8.2) has the following periodicity property: the labeled seeds $\Sigma_{m}$ and $\Sigma_{m+2(h+2)}$ are equal to each other for all $m \in \mathbb{Z}$.

(2) If $A$ is of infinite type, then all of the elements $x_{i ; m}$ in (8.9) are distinct, as are all of the elements $y_{j ; m}$ in (8.10) viewed as Laurent polynomials in the initial data.

To rephrase Theorem 8.8: the bipartite belt (or the associated $Y$-system) is periodic if and only if the Cartan matrix $A$ is of finite type; if it is periodic, then $2(h+2)$ is a period; if it is not, then all cluster variables on the bipartite belt are distinct, as are all Laurent polynomials in the associated $Y$-system.

Theorem 8.8 is proved in $\S 10$.

\section{Bipartite dynamics on roots}

As before, let $A=\left(a_{i j}\right)$ be a (symmetrizable generalized) $n \times n$ Cartan matrix with a bipartite Coxeter graph. We identify the lattice $\mathbb{Z}^{n}$ with the root lattice $Q$ associated with $A$. Specifically, the standard basis vectors in $\mathbb{Z}^{n}$ are denoted by $\alpha_{1}, \ldots, \alpha_{n}$ and identified with the simple roots. Let $W \subset \mathrm{GL}(Q)$ be the corresponding Weyl group; it is generated by the simple reflections $s_{1}, \ldots, s_{n}$ which act on the simple roots by

$$
s_{i}\left(\alpha_{j}\right)=\alpha_{j}-a_{i j} \alpha_{i} .
$$

Following [FZ03b], we define the elements $t_{ \pm} \in W$ by setting

$$
t_{+}=\prod_{\varepsilon(k)=1} s_{k}, \quad t_{-}=\prod_{\varepsilon(k)=-1} s_{k} .
$$

As in (8.3), these two elements are well defined because the factors in each of the two products mutually commute. By the same reason, each of them is an involution: $t_{+}^{2}=t_{-}^{2}=1$. 


\section{S. Fomin And A. Zelevinsky}

To illustrate (9.1) and (9.2), the actions of $t_{+}$and $t_{-}$on the simple roots are given by

$$
\begin{aligned}
& t_{+}\left(\alpha_{j}\right)= \begin{cases}-\alpha_{j} & \text { if } \varepsilon(j)=+1 \\
\alpha_{j}-\sum_{i \neq j} a_{i j} \alpha_{i} & \text { if } \varepsilon(j)=-1\end{cases} \\
& t_{-}\left(\alpha_{j}\right)= \begin{cases}\alpha_{j}-\sum_{i \neq j} a_{i j} \alpha_{i} & \text { if } \varepsilon(j)=+1 \\
-\alpha_{j} & \text { if } \varepsilon(j)=-1\end{cases}
\end{aligned}
$$

Definition 9.1. We define the vectors $\alpha(i ; m) \in Q$, for all $i \in[1, n]$ and all $m \in \mathbb{Z}$ such that $\varepsilon(i)=(-1)^{m}$, by setting, for all $r \geqslant 0$,

$$
\begin{gathered}
\alpha(i ; r)=\underbrace{t_{-} t_{+} \cdots t_{\varepsilon(i)}}_{r \text { factors }}\left(-\alpha_{i}\right) \quad \text { for } \varepsilon(i)=(-1)^{r} ; \\
\alpha(j ;-r-1)=\underbrace{t_{+} t_{-} \cdots t_{\varepsilon(j)}}_{r \text { factors }}\left(-\alpha_{j}\right) \quad \text { for } \varepsilon(j)=(-1)^{r-1} .
\end{gathered}
$$

In particular, we have $\alpha(i ; m)=-\alpha_{i}$ for $m \in\{0,-1\}$. (Note that this notation is slightly different from that in [FZ03b].)

Example 9.2 [FZ03b, Example 2.3]. In type $A_{2}$ (cf. Example 8.3), the elements $t_{+}=s_{1}$ and $t_{-}=s_{2}$ act as follows:

$$
-\alpha_{1} \stackrel{t_{+}}{\longleftrightarrow} \alpha_{1} \stackrel{t_{-}}{\longleftrightarrow} \alpha_{1}+\alpha_{2} \stackrel{t_{+}}{\longleftrightarrow} \alpha_{2} \stackrel{t_{-}}{\longleftrightarrow}-\alpha_{2} .
$$

The corresponding vectors $\alpha(i ; m)$ with $m \in[-1,4]$ are shown in Table 5 .

Clearly, all of the vectors $\alpha(i ; m)$ are (real) roots; in particular, for each of them, all non-zero components are of the same sign. To determine these signs, we assume that the Cartan matrix $A$ is indecomposable; the general case easily reduces to this one.

Proposition 9.3. Let $A$ be an indecomposable Cartan matrix of finite type (i.e. one of the CartanKilling types $\left.A_{n}, \ldots, G_{2}\right)$. Let $h$ be the corresponding Coxeter number, that is, the order of the Coxeter element $t_{+} t_{-}$in $W$. Then $\alpha(i ; m)$ is a positive root for any $m \in[-h-1, h]-\{-1,0\}$ and any $i$ with $\varepsilon(i)=(-1)^{m}$. All of these positive roots are distinct, and every positive root associated to $A$ appears among them. Furthermore, we have

$$
\begin{gathered}
\alpha(i ;-h-2)=-\alpha_{i^{*}} \quad \text { for } \varepsilon(i)=(-1)^{h}, \\
\alpha(j ; h+1)=-\alpha_{j^{*}} \quad \text { for } \varepsilon(j)=(-1)^{h-1},
\end{gathered}
$$

where $i \mapsto i^{*}$ is the involution induced by the longest element $w_{0} \in W: w_{0}\left(\alpha_{i}\right)=-\alpha_{i^{*}}$.

Proof. This is an immediate consequence of a classical result of Steinberg [Ste59] (cf. [FZ03b, Lemma 2.1 and Proposition 2.5]).

Theorem 9.4. Let $A$ be an indecomposable Cartan matrix of infinite type whose Coxeter graph is bipartite. Then the vectors $\alpha(i ; m)$ (see Definition 9.1) for $m \in \mathbb{Z}-\{-1,0\}$ and $\varepsilon(i)=(-1)^{m}$ are distinct positive roots.

Proof. Consider the matrix $A^{\prime}=2-A$. Since $A^{\prime}$ is symmetrizable, all of its eigenvalues are real. Let $\rho$ denote the maximal eigenvalue of $A^{\prime}$. As in [BLM89], we observe that $\rho \geqslant 2$. Indeed, a Cartan matrix $A$ is of finite type if and only if its symmetrization is positive definite, i.e. all eigenvalues of $A$ are positive or, equivalently, all eigenvalues of $A^{\prime}$ are less than 2. (As shown in [BLM89], $A$ is of affine type if and only if $\rho=2$, but we will not need this.) 


\section{Cluster algebras IV}

We next note that all entries of $A^{\prime}$ are non-negative. Moreover, since $A$ is indecomposable, some power of $A^{\prime}$ has all entries positive. Hence, the Perron-Frobenius theorem applies to $A^{\prime}$, implying in particular that an eigenvector for the eigenvalue $\rho$ of the transpose of $A^{\prime}$ can be chosen to have all components positive. Explicitly, there are positive real numbers $c_{1}, \ldots, c_{n}$ such that

$$
-\sum_{i \neq j} a_{i j} c_{i}=\rho c_{j} \quad \text { for any } j \in[1, n] .
$$

Let $\mathbb{R}^{2}$ be a two-dimensional real vector space with a fixed basis $\left\{\mathbf{e}_{+}, \mathbf{e}_{-}\right\}$and two linear involutions $s_{+}$and $s_{-}$acting in this basis by the matrices

$$
s_{+}=\left[\begin{array}{rr}
-1 & \rho \\
0 & 1
\end{array}\right], \quad s_{-}=\left[\begin{array}{rr}
1 & 0 \\
\rho & -1
\end{array}\right] .
$$

Let $U: Q \rightarrow \mathbb{R}^{2}$ be the linear map defined by $U\left(\alpha_{i}\right)=c_{i} \mathbf{e}_{\varepsilon(i)}$ for all $i$, where $\left(c_{1}, \ldots, c_{n}\right) \in \mathbb{R}_{>0}^{n}$ satisfies (9.10).

Lemma 9.5. We have $U t_{ \pm}=s_{ \pm} U$.

Proof. The two cases are completely analogous, so we only treat one. This is a simple calculation that uses (9.3), (9.10), and (9.11) (in this order):

$$
\begin{aligned}
& U t_{+}\left(\alpha_{j}\right)= \begin{cases}-c_{j} \mathbf{e}_{+} & \text {if } \varepsilon(j)=+1 ; \\
c_{j} \mathbf{e}_{-}-\left(\sum_{i \neq j} a_{i j} c_{i}\right) \mathbf{e}_{+} & \text {if } \varepsilon(j)=-1 ;\end{cases} \\
& = \begin{cases}-c_{j} \mathbf{e}_{+} & \text {if } \varepsilon(j)=+1 ; \\
c_{j} \mathbf{e}_{-}+\rho c_{j} \mathbf{e}_{+} & \text {if } \varepsilon(j)=-1 ;\end{cases} \\
& =c_{j} s_{+}\left(\mathbf{e}_{\varepsilon(j)}\right) \\
& =s_{+} U\left(\alpha_{j}\right) \text {, }
\end{aligned}
$$

as desired.

Note that $U$ sends positive (respectively, negative) roots to positive (respectively, negative) linear combinations of $\mathbf{e}_{+}$and $\mathbf{e}_{-}$. To show that each $\alpha(i ; m)$ with $m \in \mathbb{Z}-\{-1,0\}$ and $\varepsilon(i)=(-1)^{m}$ is a positive root, it therefore suffices to prove that $U(\alpha(i ; m))$ is a positive linear combination of $\mathbf{e}_{+}$ and $\mathbf{e}_{-}$. By Lemma 9.5,

$$
U(\alpha(i ; m))=U t_{ \pm} t_{\mp} \cdots t_{\varepsilon(i)}\left(-\alpha_{i}\right)=c_{i} s_{ \pm} s_{\mp} \cdots s_{-\varepsilon(i)}\left(\mathbf{e}_{\varepsilon(i)}\right) .
$$

Thus, we need to show the following elementary statement: if $s_{+}$and $s_{-}$are given by (9.11) with $\rho \geqslant 2$, then any vector of the form $s_{ \pm} s_{\mp} \cdots s_{\varepsilon}\left(\mathbf{e}_{-\varepsilon}\right)$ is a positive linear combination of $\mathbf{e}_{+}$and $\mathbf{e}_{-}$. This statement is hardly new, and easy to prove. Direct inspection shows that the vectors in question are of the form $\left(U_{m}(\rho), U_{m+1}(\rho)\right)$ and $\left(U_{m+1}(\rho), U_{m}(\rho)\right)$ for $m>0$, where the sequence $\left(U_{m}(\rho)\right)$ is defined by the linear recurrence

$$
U_{0}(\rho)=0, \quad U_{1}(\rho)=1, \quad U_{m+1}(\rho)=\rho U_{m}(\rho)-U_{m-1}(\rho) \quad(m>0) .
$$

Assuming that $U_{m}(\rho)>U_{m-1}(\rho) \geqslant 0$ for some $m>0$ and using the fact that $\rho \geqslant 2$, we conclude that $U_{m+1}(\rho)-U_{m}(\rho) \geqslant U_{m}(\rho)-U_{m-1}(\rho)>0$, implying by induction on $m$ that $U_{m}(\rho)>0$ for all $m>0$, as desired.

We have shown that all of the roots in (9.5) and (9.6) with $r>0$, i.e. the roots $\alpha(i ; r)$ and $\alpha(j ;-r-1)$ with $r>0$, are positive. To finish the proof of Theorem 9.4, it remains to show that all of these roots are distinct. Thus, we need to show that every equality of the form

$$
t_{ \pm} t_{\mp} \cdots t_{\varepsilon(i)}\left(-\alpha_{i}\right)=t_{ \pm} t_{\mp} \cdots t_{\varepsilon(j)}\left(-\alpha_{j}\right)
$$




\section{S. Fomin And A. Zelevinsky}

implies that $i=j$ and there are the same number of factors on both sides. Let $w$ (respectively, $w^{\prime}$ ) denote the product $t_{ \pm} t_{\mp} \cdots t_{\varepsilon(i)}$ (respectively, $\left.t_{ \pm} t_{\mp} \cdots t_{\varepsilon(j)}\right)$ on the left-hand (respectively, on the right-hand) side in (9.12). Rewriting (9.12) as

$$
-\alpha_{i}=w^{-1} w^{\prime}\left(-\alpha_{j}\right)
$$

we note that, unless the products $w$ and $w^{\prime}$ are identical, the root $w^{-1} w^{\prime}\left(-\alpha_{j}\right)$ is positive as shown above, and so cannot be equal to $-\alpha_{i}$, finishing the proof.

Theorem 9.4 can be rephrased as follows.

Corollary 9.6. Let $A$ be an $n \times n$ indecomposable Cartan matrix of infinite type whose Coxeter graph is bipartite. Then $\ell\left(\left(t_{+} t_{-}\right)^{r}\right)=r n$ for all $r>0$, where $\ell(w)$ is the length of an element $w$ in the Coxeter group $W$. Equivalently, the concatenation of any number of copies of $\prod_{\varepsilon(k)=1} s_{k} \prod_{\varepsilon(k)=-1} s_{k}$ is a reduced decomposition in $W$.

Proof. The equivalence of Corollary 9.6 and Theorem 9.4 is clear from the following well-known characterization of reduced decompositions: a decomposition $s_{i_{1}} \cdots s_{i_{\ell}}$ is reduced if and only if all the roots $s_{i_{1}} \cdots s_{i_{k-1}}\left(\alpha_{i_{k}}\right)$ are positive for $k=1, \ldots, \ell$.

Remark 9.7. Corollary 9.6 sharpens the following result in [BLM89]: if $A$ is an indecomposable Cartan matrix of infinite type with a bipartite Coxeter graph, then the element $t_{+} t_{-} \in W$ is of infinite order. (In fact [How82], any Coxeter element in any infinite Coxeter group has infinite order.) The above proof of Theorem 9.4 is based on similar ideas as the proof in [BLM89] but seems to be simpler although we prove a stronger statement.

Remark 9.8. It is conceivable that Corollary 9.6 could be extended to an arbitrary infinite Coxeter group $W$ (not necessarily crystallographic) with a bipartite Coxeter graph. We note that in this generality, the Coxeter elements $t_{+} t_{-}$were studied in $[\mathrm{McM} 02, \S 5]$.

\section{Denominators and g-vectors on the bipartite belt}

We stick to the notation of the preceding two sections. In this section we compute, for any cluster variable $x_{i ; m}$ lying on the bipartite belt, the denominator vector and the g-vector of $x_{i ; m}$ with respect to the initial cluster $\mathbf{x}_{0}$.

We begin by obtaining an expression for the denominator vector of $x_{i ; m}$ (see (7.2) and (7.3)) that is similar to that in Definition 9.1.

Let $\Phi_{\geqslant-1}^{\text {re }}$ denote the union of the set of real positive roots and the set of negative simple roots. Following [FZ03b], we introduce the involutive permutations $\tau_{+}$and $\tau_{-}$of $\Phi_{\geqslant-1}^{\mathrm{re}}$ by setting

$$
\tau_{\varepsilon}(\alpha)= \begin{cases}\alpha & \text { if } \alpha=-\alpha_{j} \text { with } \varepsilon(j)=-\varepsilon \\ t_{\varepsilon}(\alpha) & \text { otherwise }\end{cases}
$$

$($ see $(9.2))$.

Example 10.1. In type $A_{2}$ (cf. Example 9.2), the actions of $\tau_{+}$and $\tau_{-}$on $\Phi_{\geqslant-1}^{\mathrm{re}}$ are given by the following.

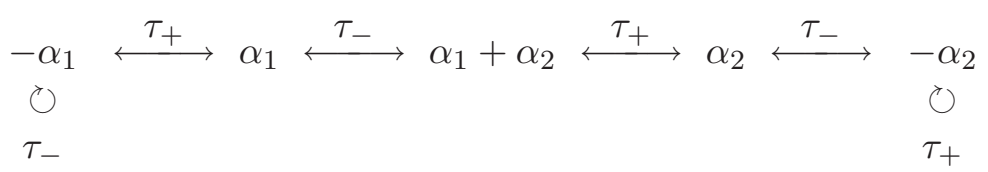




\section{Cluster algebras IV}

Definition 10.2 (cf. Definition 9.1). We define the vectors $\mathbf{d}(i ; m) \in Q$, for all $i \in[1, n]$ and all $m \in \mathbb{Z}$ such that $\varepsilon(i)=(-1)^{m}$, by setting, for all $r \geqslant 0$ :

$$
\begin{gathered}
\mathbf{d}(i ; r)=\underbrace{\tau_{-} \tau_{+} \cdots \tau_{\varepsilon(i)}}_{r \text { factors }}\left(-\alpha_{i}\right) \quad \text { for } \varepsilon(i)=(-1)^{r} ; \\
\mathbf{d}(j ;-r-1)=\underbrace{\tau_{+} \tau_{-} \cdots \tau_{\varepsilon(j)}}_{r \text { factors }}\left(-\alpha_{j}\right) \quad \text { for } \varepsilon(j)=(-1)^{r-1} .
\end{gathered}
$$

In particular, $\mathbf{d}(i ; m)=-\alpha_{i}$ for $m \in\{0,-1\}$.

TheOREM 10.3. The denominator vector for a cluster variable $x_{i ; m}$ with respect to the initial cluster $\mathbf{x}_{0}$ is equal to $\mathbf{d}(i ; m)$, for any $i \in[1, n]$ and $m \in \mathbb{Z}$ with $\varepsilon(i)=(-1)^{m}$.

Proof. It suffices to treat the case when the Cartan matrix $A$ is indecomposable. We start by clarifying the relationship between the vectors $\mathbf{d}(i ; m)$ and $\alpha(i ; m)$. First suppose that $A$ is of finite type. Comparing Definitions 9.1 and 10.2 and using Proposition 9.3, we conclude that in this case

$$
\mathbf{d}(i ; m)=\alpha(i ; m) \quad(m \in[-h-2, h+1]),
$$

and

$$
\mathbf{d}(i ; m+2(h+2))=\mathbf{d}(i ; m) \quad(m \in \mathbb{Z}) .
$$

If $A$ is of infinite type then by Theorem 9.4

$$
\mathbf{d}(i ; m)=\alpha(i ; m) \quad(m \in \mathbb{Z}) .
$$

Turning to the proof of Theorem 10.3, note that it holds for $m \in\{-1,0\}$ since in this case, for $\varepsilon(i)=(-1)^{m}$, the denominator vector of $x_{i ; m}$ is equal to $\mathbf{d}(i ; m)=-\alpha_{i}$. Applying (7.7) to the exchange relations (8.12), we see that to finish the proof it suffices to show that the vectors $\mathbf{d}(i ; m)$ with $\varepsilon(i)=(-1)^{m}$ satisfy the recurrence relation

$$
\mathbf{d}(j ; m-1)+\mathbf{d}(j ; m+1)=\left[-\sum_{\varepsilon(i)=-\varepsilon(j)} a_{i j} \mathbf{d}(i ; m)\right]_{+}
$$

(recall that the operation $a \mapsto[a]_{+}$on vectors is understood component-wise).

First suppose that $A$ is of infinite type, and so $\mathbf{d}(i ; m)=\alpha(i ; m)$ for all $m \in \mathbb{Z}$. The equality (10.8) holds for $m=0$ since $\mathbf{d}(j ; 1)=\alpha(j ; 1)=\alpha_{j}$ for $\varepsilon(j)=-1$. For $m>0$, all roots $\alpha(i ; m)$ are positive by Theorem 9.4, hence (10.8) takes the form

$$
\alpha(j ; m-1)+\alpha(j ; m+1)=-\sum_{\varepsilon(i)=-\varepsilon(j)} a_{i j} \alpha(i ; m) .
$$

The equality (10.9) is a direct consequence of (9.5):

$$
\begin{aligned}
-\alpha(j ; m-1)-\sum_{\varepsilon(i)=(-1)^{m}} a_{i j} \alpha(i ; m) & =\underbrace{t_{-} t_{+} t_{-} \cdots}_{m-1 \text { factors }}\left(\alpha_{j}-\sum_{\varepsilon(i)=(-1)^{m}} a_{i j} \alpha_{i}\right) \\
& =\underbrace{t_{-} t_{+} t_{-} \cdots}_{m \text { factors }}\left(\alpha_{j}\right) \\
& =\alpha(j ; m+1) .
\end{aligned}
$$

The case $m<0$ is treated in the same way.

If $A$ is of finite type, we use (10.5), and then the same argument as above proves (10.8) for $m \in[-h-1, h]$. The cases $m=-h-2$ and $m=h+1$ follow from (9.8) and (9.9); for $m$ outside the interval $[-h-2, h+1]$, use the periodicity property (10.6). 


\section{S. Fomin And A. Zelevinsky}

Example 10.4. To illustrate Theorem 10.3, the denominator vectors for the elements $x_{i ; m}$ shown in Table 5 appear in the last column of that table. In type $A_{2}$, we have $h=3$, so for all of the values shown in the last column, (10.5) applies.

As a first corollary of Theorem 10.3, we show that Conjecture 7.5 holds for the clusters on the bipartite belt.

Corollary 10.5. For any $m \in \mathbb{Z}$, the denominator vectors of the cluster variables

$$
\left\{x_{j ; m-1}: \varepsilon(j)=(-1)^{m-1}\right\} \cup\left\{x_{i ; m}: \varepsilon(i)=(-1)^{m}\right\} .
$$

are sign-coherent (see Definition 6.12).

Proof. Combine formulas (10.5)-(10.7) with Proposition 9.3 and Theorem 9.4.

The following result generalizes its finite-type counterpart obtained in [FZ03a, Theorem 1.9].

Corollary 10.6. Each cluster variable $x_{i ; m}$ can be written as

$$
x_{i ; m}=\frac{P_{i ; m}\left(\mathbf{x}_{0}\right)}{\mathbf{x}_{0}^{\mathbf{d}(i ; m)}}
$$

where $P_{i ; m}$ is a polynomial with non-zero constant term.

Proof. The fact that $x_{i ; m}$ is a Laurent polynomial whose numerator has non-zero constant term is proved by induction on $m$ employing [FZ03a, Lemma 5.2]. The denominator is then given by Theorem 10.3.

As another application of Theorem 10.3, we compute the tropical evaluation of the coefficients $y_{j ; m}$. As in $(8.16)$, we use $\mathbf{y}_{0}=\left(y_{1}, \ldots, y_{n}\right)$ as the initial data. We use the shorthand

$$
\mathbf{y}_{0}^{\mathbf{d}}=\prod_{k \in[1, n]} y_{k}^{d_{k}} \quad \text { for } \mathbf{d}=\left[\begin{array}{c}
d_{1} \\
\vdots \\
d_{n}
\end{array}\right] \in \mathbb{Z}^{n} .
$$

Proposition 10.7. For all $m \in \mathbb{Z}$ and $j \in[1, n]$ with $\varepsilon(j)=(-1)^{m-1}$, the elements $y_{j ; m}$ evaluated in the tropical semifield $\mathbb{P}=\operatorname{Trop}\left(y_{1}, \ldots, y_{n}\right)$ are given by

$$
\left.y_{j ; m}\right|_{\operatorname{Trop}\left(y_{1}, \ldots, y_{n}\right)}=\mathbf{y}_{0}^{-\mathbf{d}(j ; m-1)}
$$

(see Definition 10.2).

Proof. We first verify (10.10) by inspection for $m \in\{-1,0\}$. Assuming (10.10) for some $m \in \mathbb{Z}$, we easily see from the definition of the tropical semifield and the sign coherence property of Corollary 10.5 that the right-hand side of (8.11) takes the form

$$
\prod_{\varepsilon(j)=(-1)^{m-1}}\left(y_{j ; m} \oplus 1\right)^{-a_{j i}}=\left(\prod_{\varepsilon(j)=(-1)^{m-1}} y_{j ; m}^{-a_{j i}}\right) \oplus 1 .
$$

As a result, the $Y$-system dynamics (8.11) becomes identical to the cluster dynamics (8.12) (with trivial coefficients) after identifying $y_{j ; m}$ with $x_{j ; m-1}$. Thus, $(10.10)$ can be seen as a special case of Theorem 10.3.

Example 10.8. In type $A_{2}$, the monomials $\left.y_{j ; m}\right|_{\operatorname{Trop}\left(y_{1}, y_{2}\right)}$ already appeared in Table 2 . Here we are only interested in the values $y_{j ; m}$ with $j \equiv m \bmod 2$. See the second column of Table 6 . The corresponding vectors of exponents match the entries located one row higher in the fourth column of Table 6 (or, equivalently, the last column of Table 5). 


\section{Cluster algebras IV}

TABLE 6. Bipartite dynamics with principal coefficients in type $A_{2}$.

\begin{tabular}{|c|c|c|c|c|c|c|c|c|}
\hline$m$ & $y_{1 ; m}$ & $y_{2 ; m}$ & $x_{1 ; m}$ & $x_{2 ; m}$ & $\mathbf{d}(1 ; m)$ & $\mathbf{d}(2 ; m)$ & $\mathbf{g}(1 ; m)$ & $\mathbf{g}(2 ; m)$ \\
\hline-1 & $\frac{1}{y_{1}}$ & & & $x_{2}$ & & $-\alpha_{2}$ & & $\alpha_{2}$ \\
\hline 0 & & $y_{2}$ & $x_{1}$ & & $-\alpha_{1}$ & & $\alpha_{1}$ & \\
\hline 1 & $y_{1}$ & & & $\frac{x_{1} y_{2}+1}{x_{2}}$ & & $\alpha_{2}$ & & $-\alpha_{2}$ \\
\hline 2 & & $\frac{1}{y_{2}}$ & $\frac{x_{1} y_{1} y_{2}+y_{1}+x_{2}}{x_{1} x_{2}}$ & & $\alpha_{1}+\alpha_{2}$ & & $-\alpha_{1}$ & \\
\hline 3 & $\frac{1}{y_{1} y_{2}}$ & & & $\frac{y_{1}+x_{2}}{x_{1}}$ & & $\alpha_{1}$ & & $-\alpha_{1}+\alpha_{2}$ \\
\hline 4 & & $\frac{1}{y_{1}}$ & $x_{2}$ & & $-\alpha_{2}$ & & $\alpha_{2}$ & \\
\hline 5 & $y_{2}$ & & & $x_{1}$ & & $-\alpha_{1}$ & & $\alpha_{1}$ \\
\hline
\end{tabular}

Proposition 10.7 enables us to explicitly write the exchange relations (8.12) in the cluster algebra $\mathcal{A}_{\text {. }}(B)$ with principal coefficients at the initial bipartite seed $\left(\mathbf{x}_{0}, \mathbf{y}_{0}, B\right)$ (see Definitions 3.1 and 8.1) in terms of the denominators $\mathbf{d}(i ; m)$.

Corollary 10.9. In $\mathcal{A}_{\bullet}(B)$, the exchange relations (8.12) take the form

$$
x_{j ; m-1} x_{j ; m+1}=\mathbf{y}_{0}^{[-\mathbf{d}(j ; m-1)]_{+}} \prod_{\varepsilon(i)=-\varepsilon(j)} x_{i ; m}^{-a_{i j}}+\mathbf{y}_{0}^{[\mathbf{d}(j ; m-1)]_{+}},
$$

for $m \in \mathbb{Z}$ and $\varepsilon(j)=(-1)^{m-1}$.

Corollary 10.10. Conjectures 5.4, 5.5, and 7.17 (hence, Conjecture 6.11) hold in the case when the clusters and cluster variables in question belong to the bipartite belt. Thus, a polynomial $F_{\ell ; t_{m}}^{B^{0} t_{0}}$ has constant term 1 , and among its monomials there is one, namely $\mathbf{y}_{0}^{[\mathbf{d}(\ell ; m)]_{+}}$, which is divisible by all others.

Proof. Conjectures 5.4 and 5.5 follow from the observation that the formula (10.11) satisfies condition (iii) of Proposition 5.6. It remains to show (7.20). This is also an easy consequence of (10.11), which implies that the denominator vectors $\mathbf{d}(\ell ; m)$ and the tropical evaluations in (7.20) satisfy the same recurrence relations (we leave the details to the reader).

Remark 10.11. Tropical evaluations of the elements $y_{j ; m}$ in Proposition 10.7 can be viewed as $Y$-system analogues of the denominator vectors of cluster variables. Indeed, by Theorem 8.5, every element $y_{j ; m}$ with $\varepsilon(j)=(-1)^{m-1}$ is an integer Laurent polynomial in the initial data $y_{1}, \ldots, y_{n}$. In analogy with (7.2), Proposition 10.7 can be rephrased as saying that this Laurent polynomial has the form

$$
y_{j ; m}=\frac{N\left(y_{1}, \ldots, y_{n}\right)}{\mathbf{y}_{0}^{\mathbf{d}(j ; m-1)}},
$$

where $N$ is an integer polynomial not divisible by any of its $n$ variables. Thus, Proposition 10.7 provides a 'denominator vector' parameterization of the elements $y_{j ; m}$ in a $Y$-system by the roots in $\Phi_{\geqslant-1}^{\mathrm{re}}$ contained in the $\left\langle\tau_{+}, \tau_{-}\right\rangle$-orbit of the negative simple roots. This parameterization is close to but different from that of the elements in a $Y$-system (by the same set of roots) used in [FZ03b] 


\section{S. Fomin And A. Zelevinsky}

in the finite type situation. The relationship between these two parameterizations will be discussed in the next section.

Proof of Theorem 8.8. First suppose that an indecomposable Cartan matrix $A$ is of infinite type. Combining (10.7) with Theorem 9.4, we conclude that the vectors $\mathbf{d}(i ; m)$ for $m \in \mathbb{Z}$ and $\varepsilon(i)=$ $(-1)^{m}$ are all distinct. Therefore, the cluster variables $x_{i ; m}$ (respectively, the Laurent polynomials $y_{i ; m+1}$ ) are all distinct by Theorem 10.3 (respectively, by Proposition 10.7), proving part (2) of Theorem 8.8.

Now suppose that $A$ is of finite type. In view of (8.17), to prove part (1) of Theorem 8.8, one only needs to show that $x_{i ; m}=x_{i ; m+2(h+2)}$ for all $i$ and $m$ with $\varepsilon(i)=(-1)^{m}$. This follows from Theorem 10.3, the periodicity property (10.6), and the following result, which is a consequence of [FZ03a, Theorem 1.9] (see also Proposition 11.1 below): in a cluster algebra of finite type, a cluster variable is uniquely determined by its denominator vector with respect to a bipartite initial cluster $\mathbf{x}_{0}$.

Proof of Proposition 4.9. The 'only if' part is immediate from the fact (established in [FZ03a] and already mentioned in $\S 4)$ that the property of a seed $(\mathbf{x}, \mathbf{y}, B)$ to define a cluster algebra of finite type depends only on the matrix $B$. To prove the 'if' part, we need to show the following.

(*) If $B$ gives rise to a cluster algebra of infinite type, then there exists a matrix $\tilde{B}$ with principal part $B$ such that $\tilde{B}$ is of infinite mutation type.

As shown in [FZ03a], if $B$ gives rise to a cluster algebra of infinite type, then the same is true for some principal $2 \times 2$ submatrix $B^{\prime}$ of a matrix obtained from $B$ by a sequence of mutations. It is then enough to show that $(*)$ holds for $B^{\prime}$. Since $B^{\prime}$ is bipartite, we can take the corresponding $\tilde{B}^{\prime}$ as a $4 \times 2$ matrix giving rise to the cluster algebra with principal coefficients; the fact that $\tilde{B}^{\prime}$ is of infinite mutation type is immediate from Proposition 10.7 and Theorem 9.4.

Our next goal is to compute the g-vectors of the cluster variables $x_{i ; m}$. To state the result, we denote by $E$ the linear automorphism of the root lattice $Q$ acting on the basis of simple roots by

$$
E\left(\alpha_{i}\right)=-\varepsilon(i) \alpha_{i} \quad \text { for } i \in[1, n] .
$$

Theorem 10.12. For every $i \in[1, n]$ and $m \in \mathbb{Z}$ with $\varepsilon(i)=(-1)^{m}$, the $\mathbf{g}$-vector $\mathbf{g}(i ; m)$ of the cluster variable $x_{i ; m}$ with respect to the initial seed is given by

$$
\mathbf{g}(i ; m)=E \tau_{-}(\mathbf{d}(i ; m))
$$

(see (10.12), (10.1), and Definition 10.2).

Proof. The desired equality (10.13) is easily checked for $m \in\{-1,0\}$. Indeed, for $\varepsilon(j)=-1$, we have

$$
E \tau_{-}(\mathbf{d}(j ;-1))=E \tau_{-}\left(-\alpha_{j}\right)=E \alpha_{j}=\alpha_{j}=\mathbf{g}(j ;-1) ;
$$

and, for $\varepsilon(i)=+1$, we have

$$
E \tau_{-}(\mathbf{d}(i ; 0))=E \tau_{-}\left(-\alpha_{i}\right)=E\left(-\alpha_{i}\right)=\alpha_{i}=\mathbf{g}(i ; 0) .
$$

To prove (10.13) for the rest of the values of $m$, recall (7.15) and (6.4). The vector $\mathbf{g}(i ; m)$ is equal to the degree of $x_{i ; m}$ in $\mathcal{A}_{\bullet}(B)$. In view of Corollary 10.9 , for any $m \in \mathbb{Z}$ and $\varepsilon(j)=(-1)^{m-1}$, we have

$$
\mathbf{g}(j ; m-1)+\mathbf{g}(j ; m+1)=\operatorname{deg}\left(\mathbf{y}_{0}^{[\mathbf{d}(j ; m-1)]_{+}}\right) .
$$




\section{Cluster algebras IV}

Remembering the definition (6.1), we rewrite the right-hand side of (10.14) as follows:

$$
\begin{aligned}
\operatorname{deg}\left(\mathbf{y}_{0}^{[\mathbf{d}(j ; m-1)]_{+}}\right) & =\sum_{k}\left[\mathbf{d}(j ; m-1)_{k}\right]_{+} \operatorname{deg}\left(y_{k}\right) \\
& =\sum_{k}\left[\mathbf{d}(j ; m-1)_{k}\right]_{+} \sum_{i \neq k} \varepsilon(i) a_{i k} \alpha_{i},
\end{aligned}
$$

where $\mathbf{d}(j ; m-1)_{k}$ is the $k$ th component of the vector $\mathbf{d}(j ; m-1)$.

To simplify (10.15) further, we follow [FZ03b, (1.7)] and extend the permutations $\tau_{+}$and $\tau_{-}$of $\Phi_{\geqslant-1}^{\mathrm{re}}$ to piecewise-linear involutions of the root lattice $Q$ by setting

$$
\tau_{\varepsilon}\left(c_{1} \alpha_{1}+\cdots+c_{n} \alpha_{n}\right)=c_{1}^{\prime} \alpha_{1}+\cdots+c_{n}^{\prime} \alpha_{n},
$$

where

$$
c_{i}^{\prime}= \begin{cases}-c_{i}-\sum_{j \neq i} a_{i j}\left[c_{j}\right]_{+} & \text {if } \varepsilon(i)=\varepsilon ; \\ c_{i} & \text { otherwise. }\end{cases}
$$

As an immediate consequence of (10.16) and (10.17), we have, for any $\alpha=c_{1} \alpha_{1}+\cdots+c_{n} \alpha_{n} \in Q$,

$$
\tau_{+} \alpha+\tau_{-} \alpha=-\sum_{i=1}^{n} \sum_{j \neq i} a_{i j}\left[c_{j}\right]_{+} \alpha_{i} .
$$

Using (10.18), we rewrite (10.15) as follows:

$$
\operatorname{deg}\left(\mathbf{y}_{0}^{[\mathbf{d}(j ; m-1)]_{+}}\right)=E\left(\tau_{-} \mathbf{d}(j ; m-1)+\tau_{+} \mathbf{d}(j ; m-1)\right) .
$$

In view of $(10.3)$ and (10.4), we have $\mathbf{d}(j ; m-1)=\tau_{+} \tau_{-} \mathbf{d}(j ; m+1)$. Hence, (10.14) becomes

$$
\mathbf{g}(j ; m-1)+\mathbf{g}(j ; m+1)=E\left(\tau_{-} \mathbf{d}(j ; m-1)+\tau_{-} \mathbf{d}(j ; m+1)\right) .
$$

It follows that each of the equalities $\mathbf{g}(j ; m-1)=E \tau_{-}(\mathbf{d}(j ; m-1))$ and $\mathbf{g}(j ; m+1)=$ $E \tau_{-}(\mathbf{d}(j ; m+1))$ implies the other. Since we have already checked these equalities for two consecutive values of $m$, they are true for all $m \in \mathbb{Z}$, as desired.

Example 10.13. In type $A_{2}$ (with the conventions (8.13)), the maps $\tau_{ \pm}$are given by

$$
\begin{aligned}
& \tau_{+}\left(c_{1} \alpha_{1}+c_{2} \alpha_{2}\right)=\left(-c_{1}+\left[c_{2}\right]_{+}\right) \alpha_{1}+c_{2} \alpha_{2}, \\
& \tau_{-}\left(c_{1} \alpha_{1}+c_{2} \alpha_{2}\right)=c_{1} \alpha_{1}+\left(-c_{2}+\left[c_{1}\right]_{+}\right) \alpha_{2},
\end{aligned}
$$

in agreement with (10.2). The linear map $E$ acts by

$$
E\left(\alpha_{1}\right)=-\alpha_{1}, \quad E\left(\alpha_{2}\right)=\alpha_{2} .
$$

For the corresponding cluster algebra $\mathcal{A}_{\bullet}(B)$, the elements $x_{i ; m}$ and $y_{j ; m}$ are shown in Table 6 . These entries have been imported from Table 2 (or set $u_{1}=y_{1}^{-1}$ and $u_{2}=y_{2}$ in Table 5 and calculate in $\left.\operatorname{Trop}\left(y_{1}, y_{2}\right)\right)$. The denominators $\mathbf{d}(i ; m)$ and degrees $\mathbf{g}(i ; m)$ are copied from Table 4 . (Recall that, by convention, $\alpha_{1}=\left[\begin{array}{l}1 \\ 0\end{array}\right], \alpha_{2}=\left[\begin{array}{l}0 \\ 1\end{array}\right]$.)

Remark 10.14. Theorem 10.12 and Corollary 10.5 imply that Conjecture 6.13 holds in the case when the clusters and cluster variables in question lie on the bipartite belt.

By Theorems 10.3 and 10.12, the denominator vector $\mathbf{d}$ and the $\mathbf{g}$-vector $\mathbf{g}$ of any cluster variable $x_{i ; m}$ with respect to the initial seed are related by $\mathbf{g}=E \tau_{-}(\mathbf{d})$. We now show that this relationship can be extended to all cluster monomials on the bipartite belt. 


\section{S. Fomin And A. Zelevinsky}

Corollary 10.15. For every cluster monomial of the form $x_{1 ; m}^{a_{1}} \cdots x_{n ; m}^{a_{n}}$, its $\mathbf{g}$-vector $\mathbf{g}$ and its denominator vector $\mathbf{d}$ with respect to the initial seed are related by

$$
\mathbf{g}=E \tau_{-}(\mathbf{d}) .
$$

(Here $\tau_{-}$is the piecewise linear involution of $Q$ given by (10.16) and (10.17).)

Proof. In view of (7.4) and (7.14), it is enough to show that

$$
\tau_{-}\left(\sum_{i} a_{i} \mathbf{d}(i ; m)\right)=\sum_{i} a_{i} \tau_{-}(\mathbf{d}(i ; m))
$$

for any non-negative integers $a_{1}, \ldots, a_{n}$. This follows from the definition (10.16) and (10.17), once we remember Corollary 10.5.

The last result in this section provides supporting evidence for Conjectures 6.10 and 7.12 in a bipartite setting.

Proposition 10.16. Let $\Sigma_{0}$ and $\Sigma_{1}=\mu_{\varepsilon}\left(\Sigma_{0}\right)$ be adjacent seeds on the bipartite belt. Then the g-vectors

$$
\mathbf{g}(\ell ; m)=\left[\begin{array}{c}
g_{1} \\
\vdots \\
g_{n}
\end{array}\right] \quad \text { and } \quad \mathbf{g}^{\prime}(\ell ; m)=\left[\begin{array}{c}
g_{1}^{\prime} \\
\vdots \\
g_{n}^{\prime}
\end{array}\right]
$$

of a cluster variable $x_{\ell ; m}$ (with $\left.\varepsilon(\ell)=(-1)^{m}\right)$ computed with respect to the seeds $\Sigma_{0}$ and $\Sigma_{1}$, respectively, are related by

$$
g_{i}= \begin{cases}-g_{i}^{\prime} & \text { if } \varepsilon(i)=\varepsilon \\ g_{i}^{\prime}+\sum_{\varepsilon(k)=\varepsilon}\left(\left[-b_{i k}^{0}\right]_{+} g_{k}^{\prime}-b_{i k}^{0}\left[-g_{k}^{\prime}\right]_{+}\right), & \text {if } \varepsilon(i)=-\varepsilon .\end{cases}
$$

Here, as before, $\left(b_{i j}^{0}\right)$ is the exchange matrix at $\Sigma_{0}$.

Proof. It follows from the definitions combined with Theorem 10.12 that

$$
\begin{gathered}
\mathbf{g}(\ell ; m)=E \tau_{-} \mathbf{d}(\ell ; m), \\
\mathbf{g}^{\prime}(\ell ; m)=-E \tau_{+} \mathbf{d}(\ell ;-m) .
\end{gathered}
$$

(If $B$ and $B^{\prime}$ are the exchange matrices at $\Sigma_{0}$ and $\Sigma_{1}$, then $B^{\prime}=-B$, hence the corresponding sign function $\varepsilon^{\prime}:[1, n] \rightarrow\{1,-1\}$ is equal to $-\varepsilon$; this is why, in passing from (10.21) to $(10.22)$, $\tau_{-}$gets replaced by $\tau_{+}$and $E$ gets replaced by $-E$.) Definition 10.2 implies that $\mathbf{d}(\ell ; m)=\tau_{-} \mathbf{d}(\ell ;-m)$. Consequently,

$$
\mathbf{g}(\ell ; m)=E \mathbf{d}(\ell ;-m)=E \tau_{+} E\left(-\mathbf{g}^{\prime}(\ell ; m)\right) .
$$

Using (10.12), (10.17), and (8.1), this can be checked to match (10.20).

\section{Finite type}

In this section, we focus on the cluster algebras of finite type. By the classification result of [FZ03a], any cluster algebra of finite type has a bipartite seed, so all of the results in $\S \S 8-10$ apply. In fact, a cluster algebra $\mathcal{A}=\mathcal{A}\left(\mathbf{x}_{0}, \mathbf{y}_{0}, B\right)$ with a bipartite initial matrix $B$ is of finite type if and only if the Cartan counterpart $A=A(B)$ (see (8.2)) is a Cartan matrix of finite type. (The 'only if' direction follows from [FZ03a, Propositions 9.3 and 9.7].)

The results in [FZ03a, FZ03b] imply that things are particularly nice in finite type. 


\section{Cluster algebras IV}

Proposition 11.1. In a cluster algebra of finite type:

(1) every cluster variable belongs to a seed lying on the bipartite belt;

(2) the denominator vectors establish a bijection between cluster variables and 'almost positive roots' (the elements of $\Phi_{\geqslant-1}$ ), and between cluster monomials and the root lattice.

Proof. The first statement is a consequence of Proposition 9.3 and [FZ03a, Theorems 1.9 and 1.13]. To obtain the second statement, combine these theorems with [FZ03b, Theorems 1.8 and 1.10].

We now establish Conjecture 7.2 for the cluster algebras of finite type.

TheOREm 11.2. In a cluster algebra $\mathcal{A}$ of finite type, the cluster monomials are linearly independent over the ground ring $\mathbb{Z} \mathbb{P}$.

Proof. By Corollary 10.6, each cluster monomial has a unique 'leading' term of smallest total degree with respect to the cluster variables from the initial cluster. Then Proposition 11.1 implies that different cluster monomials have different leading terms, hence are linearly independent.

In [FZ04, Conjecture 4.28], we conjectured that Theorem 11.2 can be strengthened as follows: in a cluster algebra of finite type, the cluster monomials form an additive basis. For cluster algebras of infinite type, we expect this property to fail.

We next verify several conjectures from the previous sections in the special case where $\mathcal{A}$ is a cluster algebra of finite type and the initial seed $\Sigma_{0}=\Sigma_{t_{0}}$ is bipartite.

Conjecture 7.4 (hence, Conjecture 7.5, already established in more general setting - see Corollary 10.5) is immediate from [FZ03a, Theorems 1.9 and 1.13].

As an application of Proposition 11.1, we extend Corollary 10.15 to all cluster monomials (not necessarily associated with clusters lying on the bipartite belt).

Proposition 11.3. In a cluster algebra of finite type, the $\mathbf{g}$-vector $\mathbf{g}$ and the denominator vector d of any cluster monomial with respect to a bipartite initial seed are related by (10.19), where the piecewise linear involution $\tau_{-}$and the linear transformation $E$ are given by (10.16), (10.17) and (10.12).

Proof. In view of Proposition 11.1 and Theorem 10.12, the equality (10.19) holds for any cluster variable. It then extends to all cluster monomials by the same reasoning as in Corollary 10.15, using Corollary 10.5 .

We now turn to Conjectures 7.6 and 7.10. In finite type, they can be strengthened as follows.

Corollary 11.4. For a bipartite seed $\Sigma_{t_{0}}$ in a cluster algebra of finite type, each of the maps $x_{\mathbf{a} ; t} \mapsto \mathbf{d}_{\mathbf{a} ; t}^{B ; t_{0}}$ and $x_{\mathbf{a} ; t} \mapsto \mathbf{g}_{\mathbf{a} ; t}^{B ; t_{0}}$ is a bijection between the set of all cluster monomials and $\mathbb{Z}^{n}$.

Proof. The statement about denominator vectors follows from [FZ03a, Theorems 1.9 and 1.13] and [FZ03b, Theorems 1.8 and 1.10]. It implies the statement about the g-vectors in view of Proposition 11.3.

We next turn our attention to the polynomials $F_{\ell ; t}^{B ; t_{0}}$, under the assumption that $B$ is of finite type, and both seeds $t_{0}$ and $t$ lie on the bipartite belt. We use the shorthand

$$
F_{\ell ; m}=F_{\ell ; t_{m}}^{B ; t_{0}}
$$

where $t_{m}(m \in \mathbb{Z})$ is a vertex on the bipartite belt with an attached seed $\Sigma_{m}=\Sigma_{t_{m}}$ (see (8.4) and (8.5)).

We establish a link between the polynomials $F_{\ell ; m}$ and the 'Fibonacci polynomials' introduced and studied in [FZ03b]. 


\section{S. Fomin And A. Zelevinsky}

TABLE 7 . Fibonacci polynomials in type $A_{2}$.

\begin{tabular}{c|cc|cc|cc}
\hline$m$ & $F_{1 ; m}$ & $F_{2 ; m}$ & $\mathbf{d}(1 ; m)$ & $\mathbf{d}(2 ; m)$ & $f_{1 ; m}$ & $f_{2 ; m}$ \\
\hline 0 & 1 & & $-\alpha_{1}$ & & 1 & \\
1 & & $y_{2}+1$ & & $\alpha_{2}$ & & $y_{2}+1$ \\
2 & $y_{1} y_{2}+y_{1}+1$ & & $\alpha_{1}+\alpha_{2}$ & & $y_{1}+y_{2}+1$ & \\
3 & & $y_{1}+1$ & & $\alpha_{1}$ & & $y_{1}+1$ \\
4 & 1 & & $-\alpha_{2}$ & & 1 & \\
5 & & 1 & & $-\alpha_{1}$ & & 1 \\
\hline
\end{tabular}

Definition 11.5 (Fibonacci polynomials). For $\varepsilon \in\{+,-\}$, let $E_{\varepsilon}$ denote the automorphism of the field $\mathbb{Q}\left(y_{1}, \ldots, y_{n}\right)$ defined by

$$
E_{\varepsilon}\left(y_{i}\right)= \begin{cases}y_{i}^{-1} & \text { if } \varepsilon(i)=\varepsilon \\ y_{i} & \text { otherwise. }\end{cases}
$$

For $\ell \in[1, n]$ and $m \in \mathbb{Z}$, with $\varepsilon(\ell)=(-1)^{m}$, let

$$
f_{\ell ; m} \stackrel{\text { def }}{=} E_{+}\left(F_{\ell ; m}\left(y_{1}, \ldots, y_{n}\right)\right) \prod_{\varepsilon(j)=1} y_{j}^{\left[\mathbf{d}(\ell ; m)_{j}\right]_{+}},
$$

where $\mathbf{d}(\ell ; m)_{j}$ stands for the $j$ th component of the denominator vector $\mathbf{d}(\ell ; m)$. By Corollary 10.10, $f_{\ell ; m}$ is a polynomial in $y_{1}, \ldots, y_{n}$, which we call a Fibonacci polynomial.

In plain terms, $f_{\ell ; m}$ is obtained from the polynomial $F_{\ell ; m}\left(y_{1}, \ldots, y_{n}\right)$ by replacing each variable $y_{j}$ with $\varepsilon(j)=1$ by its inverse and clearing denominators. It is then clear that, conversely,

$$
F_{\ell ; m} \stackrel{\text { def }}{=} E_{+}\left(f_{\ell ; m}\left(y_{1}, \ldots, y_{n}\right)\right) \prod_{\varepsilon(j)=1} y_{j}^{\left[\mathbf{d}(\ell ; m)_{j}\right]_{+}} .
$$

For the special case of type $A_{2}$, the polynomials $F_{\ell ; m}$ and $f_{\ell ; m}$ are shown in Table 7 .

In [FZ03b, Theorem 1.6], we introduced a family of polynomials

$$
F[\alpha] \in \mathbb{Z}\left[y_{1}, \ldots, y_{n}\right]
$$

labeled by the roots $\alpha \in \Phi_{\geqslant-1}$. Combining [FZ03b, Theorem 1.5, Theorem 1.7, and Remark 2.8] and remembering Remark 8.4 and Definition 10.2 of the current paper, we see that the polynomials $F[\alpha]$ can be defined as the unique family of polynomials with the following properties. First, these polynomials satisfy the initial conditions $F\left[-\alpha_{i}\right]=1$ (for all $i$ ). Second, for any $\alpha=\mathbf{d}(j ;-m-1) \in$ $\Phi_{\geqslant-1}$ with $\varepsilon(j)=(-1)^{m-1}$, they satisfy the recurrence relation

$$
F\left[\tau_{+} \alpha\right] F\left[\tau_{-} \alpha\right]=\mathbf{y}_{0}^{[-\alpha]_{+}} \prod_{i \neq j} F[\mathbf{d}(i ; m)]^{-a_{i j}}+\mathbf{y}_{0}^{[\alpha]_{+}} .
$$

(We note that $\tau_{+} \alpha=\mathbf{d}(j ; m-1)$ and $\tau_{-} \alpha=\mathbf{d}(j ; m+1)$.)

TheOREM 11.6. In a cluster algebra of finite type, $f_{\ell ; m}=F[\alpha]$, where $\alpha=\mathbf{d}(\ell ; m)$.

Thus, the terminology of Definition 11.5 is consistent with that of [FZ03b]: the Fibonacci polynomials $f_{\ell ; m}$ and $F[\alpha]$ are indeed the same. 


\section{Cluster algebras IV}

Proof. We need to verify that the polynomials $f_{\ell ; m}$ satisfy the recurrence (11.4) (the initial conditions are immediate). As before, we use the notation $\mathbf{y}_{0}=\left(y_{1}, \ldots, y_{n}\right)$. Assuming that $\varepsilon(j)=$ $(-1)^{m-1}$, we denote

$$
\begin{aligned}
\mathbf{d}(j ; m-1) & =\left(d_{1}, \ldots, d_{n}\right), \\
\mathbf{d}(j ; m+1) & =\left(\tilde{d}_{1}, \ldots, \tilde{d}_{n}\right) .
\end{aligned}
$$

It then follows from (10.16) and (10.17) that

$$
\mathbf{d}(j ;-m-1)_{\ell}= \begin{cases}\tilde{d}_{\ell} & \text { if } \varepsilon(\ell)=1 \\ d_{\ell} & \text { if } \varepsilon(\ell)=-1\end{cases}
$$

Thus, we need to show that

$$
f_{j ; m-1} f_{j ; m+1}=\prod_{\varepsilon(\ell)=1} y_{\ell}^{\left[-\tilde{d}_{\ell}\right]_{+}} \prod_{\varepsilon(k)=-1} y_{k}^{\left[-d_{k}\right]_{+}} \prod_{i \neq j} f_{i ; m}^{-a_{i j}}+\prod_{\varepsilon(\ell)=1} y_{\ell}^{\left[\tilde{d}_{\ell}\right]_{+}} \prod_{\varepsilon(k)=-1} y_{k}^{\left[d_{k}\right]_{+}} .
$$

This can be deduced from Corollary 10.9 as follows. Combining the latter with (3.3), we get

$$
F_{j ; m-1} F_{j ; m+1}=\prod_{\ell} y_{\ell}^{\left[-d_{\ell}\right]_{+}} \prod_{\varepsilon(i)=-\varepsilon(j)} F_{i ; m}^{-a_{i j}}+\prod_{\ell} y_{\ell}^{\left[d_{\ell}\right]_{+}} .
$$

Applying $E_{+}$and using (11.2), we obtain, after straightforward simplifications,

$$
\begin{aligned}
f_{j ; m-1} f_{j ; m+1}= & \prod_{\varepsilon(\ell)=1} y_{\ell}^{d_{\ell}+\left[\tilde{d}_{\ell}\right]_{+}} \prod_{\varepsilon(k)=-1} y_{k}^{\left[-d_{k}\right]_{+}} \prod_{i \neq j}\left(f_{i ; m}^{-a_{i j}} \prod_{\varepsilon(\ell)=1} y_{\ell}^{a_{i j}\left[\mathbf{d}(i ; m)_{\ell}\right]_{+}}\right) \\
& +\prod_{\varepsilon(\ell)=1} y_{\ell}^{\left[\tilde{d}_{\ell}\right]_{+}} \prod_{\varepsilon(k)=-1} y_{k}^{\left[d_{k}\right]_{+}} .
\end{aligned}
$$

We next use (10.8), which in view of Corollary 10.5 can be rewritten as

$$
d_{\ell}+\tilde{d}_{\ell}=-\sum_{i \neq j} a_{i j}\left[\mathbf{d}(i ; m)_{\ell}\right]_{+} .
$$

Putting (11.6) and (11.7) together yields (11.5).

Corollary 11.7. Each polynomial $F_{\ell ; m}\left(y_{1}, \ldots, y_{n}\right)$ has positive coefficients.

Proof. By [FZ03b, Theorem 1.6], each polynomial $F[\alpha]$ has positive coefficients. The claim follows by Theorem 11.6 and (11.3).

In [FZ03b, § 2.4], we gave explicit formulas for the Fibonacci polynomials $F[\alpha]$ associated with the root systems of classical types $A B C D$. The Fibonacci polynomials of exceptional types can be calculated on a computer using the defining recurrence (11.2). Combining these results with (11.3), one can compute all polynomials $F_{\ell ; m}$. Corollary 6.3 then yields explicit formulas expressing an arbitrary cluster variable in an arbitrary cluster algebra of finite type in terms of the bipartite initial seed. These formulas are too bulky to be included in this paper: as mentioned in [FZ03b, p. 999], the monomial expansion of a Fibonacci polynomial of type $E_{8}$ can have up to 26908 terms.

\section{Universal coefficients}

In this section, we show that among all cluster algebras of a given finite (Cartan-Killing) type, there is one with the 'universal' system of coefficients: any other such algebra is obtained from this one by a coefficient specialization. To make this precise, we need the following definition. 


\section{S. Fomin And A. Zelevinsky}

Definition 12.1. Let $\mathcal{A}$ and $\overline{\mathcal{A}}$ be cluster algebras of the same rank $n$ over the coefficient semifields $\mathbb{P}$ and $\overline{\mathbb{P}}$, respectively, with the respective families of cluster variables $\left(x_{i ; t}\right)_{i \in[1, n], t \in \mathbb{T}_{n}}$ and $\left(\bar{x}_{i ; t}\right)_{i \in[1, n], t \in \mathbb{T}_{n}}$. We say that $\overline{\mathcal{A}}$ is obtained from $\mathcal{A}$ by a coefficient specialization if:

(1) $\mathcal{A}$ and $\overline{\mathcal{A}}$ have the same exchange matrices $B_{t}=\bar{B}_{t}$ at each vertex $t \in \mathbb{T}_{n}$;

(2) there is a homomorphism of multiplicative groups $\varphi: \mathbb{P} \rightarrow \overline{\mathbb{P}}$ that extends to a (unique) ring homomorphism $\varphi: \mathcal{A} \rightarrow \overline{\mathcal{A}}$ such that $\varphi\left(x_{i ; t}\right)=\bar{x}_{i ; t}$ for all $i$ and $t$.

With some abuse of notation, we denote both maps $\varphi: \mathbb{P} \rightarrow \overline{\mathbb{P}}$ and $\varphi: \mathcal{A} \rightarrow \overline{\mathcal{A}}$ by the same symbol, and refer to each of them as a coefficient specialization.

Recall from $\S 2$ that the cluster algebra structure is completely determined by the underlying $Y$-pattern $t \mapsto\left(\mathbf{y}_{t}, B_{t}\right)$, which is in turn completely determined by a $Y$-seed $\left(\mathbf{y}_{t_{0}}, B_{t_{0}}\right)$ attached to an arbitrary 'initial' vertex $t_{0} \in \mathbb{T}_{n}$. In the language of $Y$-patterns, Definition 12.1 can be rephrased as follows.

Proposition 12.2. Let $\mathcal{A}$ and $\overline{\mathcal{A}}$ be cluster algebras over the coefficient semifields $\mathbb{P}$ and $\overline{\mathbb{P}}$, respectively, sharing the same exchange matrices $B_{t}$. Let the underlying $Y$-patterns for $\mathcal{A}$ and $\overline{\mathcal{A}}$ be $t \mapsto\left(\mathbf{y}_{t}, B_{t}\right)$ and $t \mapsto\left(\overline{\mathbf{y}}_{t}, B_{t}\right)$, respectively. A homomorphism of multiplicative groups $\varphi: \mathbb{P} \rightarrow \overline{\mathbb{P}}$ is a coefficient specialization (that is, satisfies condition (2) in Definition 12.1) if and only if

$$
\varphi\left(y_{j ; t}\right)=\bar{y}_{j ; t}, \quad \varphi\left(y_{j ; t} \oplus 1\right)=\bar{y}_{j ; t} \oplus 1
$$

for all $j \in[1, n]$ and $t \in \mathbb{T}_{n}$.

Proof. We start by restating the condition (12.1) in terms of the coefficients appearing in the exchange relations. Following (2.7), we denote by

$$
p_{j ; t}^{+}=\frac{y_{j ; t}}{y_{j ; t} \oplus 1}, \quad p_{j ; t}^{-}=\frac{1}{y_{j ; t} \oplus 1}
$$

the two coefficients in the exchange relation for a cluster variable $x_{j ; t}$ at a vertex $t$ (cf. (2.8)); also let $\bar{p}_{j ; t}^{ \pm}$denote the counterparts of the coefficients $p_{j ; t}^{ \pm}$in the cluster algebra $\overline{\mathcal{A}}$. In view of $(12.2)$, for a homomorphism of multiplicative groups $\varphi: \mathbb{P} \rightarrow \overline{\mathbb{P}}$, condition (12.1) is equivalent to requiring

$$
\varphi\left(p_{j ; t}^{ \pm}\right)=\bar{p}_{j ; t}^{ \pm}
$$

for all $j \in[1, n]$ and $t \in \mathbb{T}_{n}$.

Let $\mathbf{x}_{0}$ and $\overline{\mathbf{x}}_{0}$ be the clusters of $\mathcal{A}$ and $\overline{\mathcal{A}}$ at some initial vertex $t_{0}$. Since the elements of $\mathbf{x}_{0}$ are algebraically independent over the ground ring $\mathbb{Z} \mathbb{P}$, any homomorphism of multiplicative groups $\varphi: \mathbb{P} \rightarrow \overline{\mathbb{P}}$ extends to a (unique) ring homomorphism $\varphi: \mathbb{Z} \mathbb{P}\left[\mathbf{x}_{0}^{ \pm 1}\right] \rightarrow \mathbb{Z} \overline{\mathbb{P}}\left[\overline{\mathbf{x}}_{0}^{ \pm 1}\right]$ by setting $\varphi\left(x_{i ; t_{0}}\right)=\bar{x}_{i ; t_{0}}$ for all $i$. Recall that by Theorem 3.5, $\mathcal{A} \subset \mathbb{Z} \mathbb{P}\left[\mathbf{x}_{0}^{ \pm 1}\right]$ and $\overline{\mathcal{A}} \subset \mathbb{Z} \overline{\mathbb{P}}\left[\overline{\mathbf{x}}_{0}^{ \pm 1}\right]$. In order to express a cluster variable at any vertex $t_{1}$ as a Laurent polynomial in the elements of the initial cluster at $t_{0}$, one only needs to iterate the exchange relations of the form (2.4) along the path connecting $t_{0}$ and $t_{1}$. Since the elements of every cluster are algebraically independent, the condition that $\varphi\left(x_{i ; t}\right)=\bar{x}_{i ; t}$ for all $i$ and $t$ is equivalent to the property that $\varphi$ sends every relation (2.4) in $\mathcal{A}$ to the corresponding relation in $\overline{\mathcal{A}}$, that is, satisfies the equivalent conditions (12.1) and (12.3).

One way to get a coefficient specialization is to take any semifield homomorphism $\varphi: \mathbb{P} \rightarrow \overline{\mathbb{P}}$. For example, if $\mathcal{A}$ is a cluster algebra of geometric type over a semifield $\operatorname{Trop}\left(u_{j}: j \in J\right)$, then for any subset $I \subset J$, there is a semifield homomorphism $\varphi: \operatorname{Trop}\left(u_{j}: j \in J\right) \rightarrow \operatorname{Trop}\left(u_{j}: j \in I\right)$ defined by setting $\varphi\left(u_{j}\right)=u_{j}$ for $j \in I$ and $\varphi\left(u_{j}\right)=1$ for $j \notin I$.

Definition 12.3. We say that a cluster algebra $\mathcal{A}$ (and the corresponding $Y$-pattern $t \mapsto\left(\mathbf{y}_{t}, B_{t}\right)$ ) has universal coefficients if every cluster algebra with the same family of exchange matrices $\left(B_{t}\right)$ is obtained from $\mathcal{A}$ by a unique coefficient specialization. 


\section{Cluster algebras IV}

By the standard universality argument, for a given family of exchange matrices $\left(B_{t}\right)$, a cluster algebra with universal coefficients is unique in the following sense: for any two such algebras $\mathcal{A}$ and $\overline{\mathcal{A}}$, there is a canonical isomorphism of multiplicative groups of their coefficient semifields that extends to a canonical ring isomorphism $\mathcal{A} \rightarrow \overline{\mathcal{A}}$ sending all cluster variables and all coefficients in the exchange relations for $\mathcal{A}$ to their respective counterparts for $\overline{\mathcal{A}}$. (A subtle point: the definition of universal coefficients does not imply the uniqueness of auxiliary addition in the coefficient semifield.)

Note that the existence of a cluster algebra with universal coefficients is by no means clear. We prove it in the following for the finite type case. To state the result, let us again recall the main theorem of [FZ03a]: any cluster algebra $\mathcal{A}$ of finite type has an initial seed with a bipartite exchange matrix $B$ such that the Cartan counterpart $A=A(B)$ is a Cartan matrix of finite type. That is, $A$ is associated with a finite root system $\Phi$, uniquely defined by $\mathcal{A}$ (up to isomorphism).

Theorem 12.4. For any bipartite matrix $B$ such that $A=A(B)$ is a Cartan matrix of finite type, there exists a universal cluster algebra $\mathcal{A}^{\text {univ }}(B)=\mathcal{A}^{\text {univ }}(\Phi)$ having $B$ as the initial exchange matrix. Specifically, $\mathcal{A}^{\text {univ }}(B)$ can be realized as follows.

- As a coefficient semifield for $\mathcal{A}^{\text {univ }}(B)$, take

$$
\mathbb{P}^{\text {univ }}=\operatorname{Trop}\left(p\left[\alpha^{\vee}\right]: \alpha^{\vee} \in \Phi_{\geqslant-1}^{\vee}\right),
$$

the tropical semifield whose generators $p\left[\alpha^{\vee}\right]$ are labeled by the set $\Phi_{\geqslant-1}^{\vee}$ of 'almost positive coroots' (i.e. positive roots and negative simple roots in the root system dual to $\Phi$ ).

- Define the initial $Y$-seed $\left(\mathbf{y}_{0}^{\text {univ }}, B\right)$, with $\mathbf{y}_{0}^{\text {univ }}=\left(y_{1 ; 0}, \ldots, y_{n ; 0}\right)$, by

$$
y_{j ; 0}=\prod_{\alpha^{\vee} \in \Phi_{\geqslant-1}^{\vee}} p\left[\alpha^{\vee}\right]^{\varepsilon(j)\left[\alpha^{\vee}: \alpha_{j}^{\vee}\right]},
$$

where $\varepsilon$ is the sign function from (8.1), and $\left[\alpha^{\vee}: \alpha_{j}^{\vee}\right]$ stands for the coefficient of $\alpha_{j}^{\vee}$ in the expansion of the coroot $\alpha^{\vee}$ in the basis of simple coroots.

The rank 2 special case of Theorem 12.4 is implicitly contained in [FZ02, Proposition 6.4 and Remark 6.5].

Before providing a proof of the theorem, we illustrate it with an example.

Example 12.5 (Universal cluster algebra of type $A_{2}$ ). Let $B=\left[\begin{array}{rr}0 & 1 \\ -1 & 0\end{array}\right]$ as in Example 8.3. Thus, $\varepsilon(1)=1$ and $\varepsilon(2)=-1$. Since the root system $\Phi$ of type $A_{2}$ is simply-laced, we can identify the coroots with the roots. By (12.4), the universal coefficient semifield $\mathbb{P}^{\text {univ }}$ is the tropical semifield with five generators:

$$
\mathbb{P}=\operatorname{Trop}\left(p\left[-\alpha_{1}\right], p\left[-\alpha_{2}\right], p\left[\alpha_{1}\right], p\left[\alpha_{1}+\alpha_{2}\right], p\left[\alpha_{2}\right]\right)
$$

Formula (12.5) gives

$$
y_{1 ; 0}=\frac{p\left[\alpha_{1}\right] p\left[\alpha_{1}+\alpha_{2}\right]}{p\left[-\alpha_{1}\right]}, \quad y_{2 ; 0}=\frac{p\left[-\alpha_{2}\right]}{p\left[\alpha_{2}\right] p\left[\alpha_{1}+\alpha_{2}\right]} .
$$

Thus, $\mathcal{A}^{\text {univ }}(B)$ is the cluster algebra of geometric type defined by the extended exchange matrix $(\operatorname{see}(2.14))$

$$
\tilde{B}=\left[\begin{array}{rr}
0 & 1 \\
-1 & 0 \\
-1 & 0 \\
0 & 1 \\
1 & 0 \\
1 & -1 \\
0 & -1
\end{array}\right]
$$




\section{S. Fomin And A. Zelevinsky}

TABLE 8. Universal coefficients in type $A_{2}$.

\begin{tabular}{c|c|cc|cc}
\hline$t$ & \multicolumn{1}{|c|}{$B_{t}$} & $y_{1 ; t}$ & $y_{2 ; t}$ & $x_{1 ; t}$ & $x_{2 ; t}$ \\
\hline 0 & {$\left[\begin{array}{rr}0 & 1 \\
-1 & 0\end{array}\right]$} & $\frac{p\left[\alpha_{1}\right] p\left[\alpha_{1}+\alpha_{2}\right]}{p\left[-\alpha_{1}\right]}$ & $\frac{p\left[-\alpha_{2}\right]}{p\left[\alpha_{2}\right] p\left[\alpha_{1}+\alpha_{2}\right]}$ & $x\left[-\alpha_{1}\right]$ & $x\left[-\alpha_{2}\right]$ \\
1 & {$\left[\begin{array}{rr}0 & -1 \\
1 & 0\end{array}\right]$} & $\frac{p\left[\alpha_{1}\right]}{p\left[-\alpha_{1}\right] p\left[\alpha_{2}\right]}$ & $\frac{p\left[\alpha_{2}\right] p\left[\alpha_{1}+\alpha_{2}\right]}{p\left[-\alpha_{2}\right]}$ & $x\left[-\alpha_{1}\right]$ & $x\left[\alpha_{2}\right]$ \\
2 & {$\left[\begin{array}{rr}0 & 1 \\
-1 & 0\end{array}\right]$} & $\frac{p\left[-\alpha_{1}\right] p\left[\alpha_{2}\right]}{p\left[\alpha_{1}\right]}$ & $\frac{p\left[\alpha_{1}+\alpha_{2}\right]}{p\left[-\alpha_{1}\right] p\left[-\alpha_{2}\right]}$ & $x\left[\alpha_{1}+\alpha_{2}\right]$ & $x\left[\alpha_{2}\right]$ \\
3 & {$\left[\begin{array}{rr}0 & -1 \\
1 & 0\end{array}\right]$} & $\frac{p\left[\alpha_{2}\right]}{p\left[-\alpha_{2}\right] p\left[\alpha_{1}\right]}$ & $\frac{p\left[-\alpha_{1}\right] p\left[-\alpha_{2}\right]}{p\left[\alpha_{1}+\alpha_{2}\right]}$ & $x\left[\alpha_{1}+\alpha_{2}\right]$ & $x\left[\alpha_{1}\right]$ \\
4 & {$\left[\begin{array}{rr}0 & 1 \\
-1 & 0\end{array}\right]$} & $\frac{p\left[-\alpha_{2}\right] p\left[\alpha_{1}\right]}{p\left[\alpha_{2}\right]}$ & $\frac{p\left[-\alpha_{1}\right]}{p\left[\alpha_{1}\right] p\left[\alpha_{1}+\alpha_{2}\right]}$ & $x\left[-\alpha_{2}\right]$ & $x\left[\alpha_{1}\right]$ \\
5 & {$\left[\begin{array}{rr}0 & -1 \\
1 & 0\end{array}\right]$} & $\frac{p\left[-\alpha_{2}\right]}{p\left[\alpha_{2}\right] p\left[\alpha_{1}+\alpha_{2}\right]}$ & $\frac{p\left[\alpha_{1}\right] p\left[\alpha_{1}+\alpha_{2}\right]}{p\left[-\alpha_{1}\right]}$ & $x\left[-\alpha_{2}\right]$ & $x\left[-\alpha_{1}\right]$ \\
\hline
\end{tabular}

(Rows $3-7$ of the matrix $\tilde{B}$ correspond to the variables $p[\alpha]\left(\alpha \in \Phi_{\geqslant-1}\right)$ in the order listed in (12.6).) We then calculate the rest of the coefficients $y_{j ; t}$ by substituting the above expressions for $y_{1}$ and $y_{2}$ into the third column of Table 1, and computing the resulting expressions in $\mathbb{P}^{\text {univ }}$. (It is useful to note the following: if $Y$ and $Y^{\prime}$ are two disjoint monomials in the generators of $\mathbb{P}^{\text {univ }}$, then $Y / Y^{\prime} \oplus 1=1 / Y^{\prime}$.) The results are shown in the third column of Table 8 .

We conclude this example by writing the exchange relations in the universal cluster algebra of type $A_{2}$. The coefficients in each of these relations are obtained by splitting the appropriate Laurent monomial in the third column of Table 8 into its numerator and its denominator. To have a consistent notation for the five cluster variables in $\mathcal{A}^{\text {univ }}(B)$, we use the denominator parameterization: let $x[\alpha]$ denote the cluster variable whose denominator vector is $\alpha$. See the fourth column of Table 8 .

With the notation agreed upon above, the exchange relations in $\mathcal{A}^{\text {univ }}(B)$ are

$$
\begin{aligned}
x\left[-\alpha_{2}\right] x\left[\alpha_{2}\right] & =p\left[-\alpha_{2}\right] x\left[-\alpha_{1}\right]+p\left[\alpha_{2}\right] p\left[\alpha_{1}+\alpha_{2}\right], \\
x\left[-\alpha_{1}\right] x\left[\alpha_{1}+\alpha_{2}\right] & =p\left[\alpha_{1}\right] x\left[\alpha_{2}\right]+p\left[-\alpha_{1}\right] p\left[\alpha_{2}\right], \\
x\left[\alpha_{2}\right] x\left[\alpha_{1}\right] & =p\left[\alpha_{1}+\alpha_{2}\right] x\left[\alpha_{1}+\alpha_{2}\right]+p\left[-\alpha_{1}\right] p\left[-\alpha_{2}\right], \\
x\left[\alpha_{1}+\alpha_{2}\right] x\left[-\alpha_{2}\right] & =p\left[\alpha_{2}\right] x\left[\alpha_{1}\right]+p\left[-\alpha_{2}\right] p\left[\alpha_{1}\right], \\
x\left[\alpha_{1}\right] x\left[-\alpha_{1}\right] & =p\left[-\alpha_{1}\right] x\left[-\alpha_{2}\right]+p\left[\alpha_{1}\right] p\left[\alpha_{1}+\alpha_{2}\right] .
\end{aligned}
$$

These exchange relations (thus, implicitly, the algebra $\mathcal{A}^{\text {univ }}(B)$ ) have already appeared ${ }^{1}$ in [FZ02, Example 7.6].

Proof of Theorem 12.4. In the following argument, we fix the bipartite exchange matrix $B$ of finite type associated with a root system $\Phi$, and work with the class of cluster algebras $\mathcal{A}$ having $B$ attached to an initial seed. We start by taking an inventory of the exchange relations in $\mathcal{A}$.

\footnotetext{
${ }^{1}$ Correction: in [FZ02, Figure 3], the exchange relation between $y_{2}$ and $y_{5}$ contained a typo.
} 


\section{Cluster algebras IV}

By Proposition 11.1, the denominator vectors with respect to the initial cluster provide a bijection $\alpha \mapsto x[\alpha]$ between the set $\Phi_{\geqslant-1}$ of 'almost positive' roots and the set of all cluster variables in $\mathcal{A}$; furthermore, this bijection extends to a bijection $\gamma \mapsto x[\gamma]$ between the root lattice $Q$ and the set of all cluster monomials in $\mathcal{A}$. Using these bijective parameterizations, we can write every exchange relation in $\mathcal{A}$ in the form

$$
x[\beta] x\left[\beta^{\prime}\right]=p_{1} x\left[\gamma_{1}\right]+p_{2} x\left[\gamma_{2}\right],
$$

where $\beta, \beta^{\prime} \in \Phi_{\geqslant-1}, \gamma_{1}, \gamma_{2} \in Q$, and $p_{1}$ and $p_{2}$ belong to the coefficient semifield $\mathbb{P}$.

These exchange relations have the following properties.

- The pairs $\left\{\beta, \beta^{\prime}\right\}$ appearing in the left-hand sides depend only on the root system $\Phi$, that is, are the same for all choices of coefficients; see [FZ03a, Proposition 3.5 and Corollary 4.4], where such pairs are called exchangeable.

- The pair $\left\{\gamma_{1}, \gamma_{2}\right\}$ on the right is uniquely determined by $\left\{\beta, \beta^{\prime}\right\}$; so it also does not depend on the choice of the coefficient system in $\mathcal{A}$. (See [FZ03a, (5.1)], where $\gamma_{1}$ and $\gamma_{2}$ are explicitly expressed in terms of $\beta$ and $\beta^{\prime}$.)

In view of these properties, we denote the coefficients in (12.13) as follows:

$$
p_{i}=p\left(\left\{\beta, \beta^{\prime}\right\} ; \gamma_{i}\right) \quad(i=1,2) .
$$

This notation becomes slightly ambiguous if $\gamma_{1}=\gamma_{2}$. However, this can only happen if $\beta=\alpha_{i}$ and $\beta^{\prime}=-\alpha_{i}$ are the two roots of an irreducible component of type $A_{1}$ in $\Phi$, in which case $\gamma_{1}=\gamma_{2}=0$; all of the arguments below will be valid in this case as well, even if both coefficients will carry the same notation.

Among the coefficients $p\left(\left\{\beta, \beta^{\prime}\right\} ; \gamma\right)$, the following will be of special importance.

Definition 12.6. A coefficient $p\left(\left\{\beta, \beta^{\prime}\right\} ; \gamma_{1}\right)$ in (12.13) and (12.14) is called primitive if $\gamma_{2}=0$, i.e. the 'opposite' monomial $x\left[\gamma_{2}\right]$ is equal to 1 .

The following lemma provides several equivalent descriptions of exchange relations with primitive coefficients.

Lemma 12.7. For an exchange relation (12.13) the following conditions are equivalent:

(1) one of the coefficients $p\left(\left\{\beta, \beta^{\prime}\right\} ; \gamma_{1}\right)$ and $p\left(\left\{\beta, \beta^{\prime}\right\} ; \gamma_{2}\right)$ is primitive;

(2) the relation in question appears on the bipartite belt, i.e. $\left\{\beta, \beta^{\prime}\right\}=\{\mathbf{d}(j ; m-1), \mathbf{d}(j ; m+1)\}$ for some $j \in[1, n]$ and $m \in \mathbb{Z}$ (see Theorem 10.3);

(3) $\left\{\beta, \beta^{\prime}\right\}=\left\{\tau_{+} \alpha, \tau_{-} \alpha\right\}$ for some $\alpha \in \Phi_{\geqslant-1}$.

Recall that $\tau_{+}$and $\tau_{-}$appearing in condition (3) are the involutive permutations of $\Phi_{\geqslant-1}$ given by (10.1); they extend to piecewise-linear involutions of the root lattice $Q$ via (10.16) and (10.17).

Proof. The equivalence of conditions (2) and (3) is clear from the formulas (10.3) and (10.4). The implication $(2) \Rightarrow(1)$ follows from the definition of the bipartite belt. To prove $(1) \Rightarrow(2)$, we use the formula given in [FZ03a, (5.1)], which gives explicit expressions for $\gamma_{1}$ and $\gamma_{2}$ in terms of $\left\{\beta, \beta^{\prime}\right\}$. As a special case of [FZ03a, (5.1)], condition (1) is equivalent to the following: $\sigma(\beta)+\sigma\left(\beta^{\prime}\right)=0$ for some transformation $\sigma$ belonging to the group generated by $\tau_{+}$and $\tau_{-}$. Since both $\sigma(\beta)$ and $\sigma\left(\beta^{\prime}\right)$ belong to $\Phi_{\geqslant-1}$, the only way they can add up to 0 is if, say, $\sigma(\beta)=-\alpha_{j}$ and $\sigma\left(\beta^{\prime}\right)=\alpha_{j}$ for some $j$. Again remembering (10.3) and (10.4), it is easy to see that in that case, $\left\{\beta, \beta^{\prime}\right\}=$ $\{\mathbf{d}(j ; m-1), \mathbf{d}(j ; m+1)\}$ for some $m \in \mathbb{Z}$, and we are done. 


\section{S. Fomin And A. Zelevinsky}

Lemma 12.8. In the algebra $\mathcal{A}^{\text {univ }}(B)$ given by (12.4) and (12.5), the primitive coefficients are precisely the generators $p\left[\alpha^{\vee}\right]$ of $\mathbb{P}^{\text {univ }}$. More specifically, if $\left\{\beta, \beta^{\prime}\right\}=\left\{\tau_{+}(\alpha), \tau_{-}(\alpha)\right\}$ for some $\alpha \in$ $\Phi_{\geqslant-1}$ (see Lemma 12.7(3)), then the corresponding primitive coefficient in (12.13) is equal to $p\left[\alpha^{\vee}\right]$, where $\alpha^{\vee}$ is the coroot associated with a root $\alpha$.

Proof. Let us explicitly solve the $Y$-system (8.11) in the semifield $\mathbb{P}^{\text {univ }}$ defined by (12.4) with the initial conditions

$$
\begin{aligned}
y_{i ;-1} & =\prod_{\alpha^{\vee} \in \Phi_{\geqslant-1}^{\vee}} p\left[\alpha^{\vee}\right]^{-\left[\alpha^{\vee}: \alpha_{i}^{\vee}\right]} \quad(\varepsilon(i)=+1), \\
y_{j ; 0} & =\prod_{\alpha^{\vee} \in \Phi_{\geqslant}^{\vee}-1} p\left[\alpha^{\vee}\right]^{-\left[\alpha^{\vee}: \alpha_{j}^{\vee}\right]} \quad(\varepsilon(j)=-1) ;
\end{aligned}
$$

by (8.8), these conditions are equivalent to (12.5). We will need the analogue of (10.16) and (10.17) for the dual root system, which can be written as

$$
\left[\tau_{\varepsilon} \alpha^{\vee}: \alpha_{j}^{\vee}\right]= \begin{cases}-\left[\alpha^{\vee}: \alpha_{j}^{\vee}\right]-\sum_{i \neq j} a_{i j}\left[\left[\alpha^{\vee}: \alpha_{i}^{\vee}\right]\right]_{+} & \text {if } \varepsilon(j)=\varepsilon ; \\ {\left[\alpha^{\vee}: \alpha_{j}^{\vee}\right]} & \text { otherwise. }\end{cases}
$$

Using (12.16) and proceeding by induction, it is straightforward to verify that the solution of (8.11) in $\mathbb{P}^{\text {univ }}$ with the initial conditions $(12.15)$ is given by

$$
\begin{aligned}
& y_{i ;-r-1}=\prod_{\alpha^{\vee} \in \Phi_{\geqslant-1}^{\vee}} p\left[\alpha^{\vee}\right]^{-\left[\tau_{\varepsilon(i)}^{(r)} \alpha^{\vee}: \alpha_{i}^{\vee}\right]} \quad\left(\varepsilon(i)=(-1)^{r}\right), \\
& y_{j ; r}=\prod_{\alpha^{\vee} \in \Phi_{\geqslant-1}^{\vee}} p\left[\alpha^{\vee}\right]^{-\left[\tau_{\varepsilon(j)}^{(r)} \alpha^{\vee}: \alpha_{j}^{\vee}\right]} \quad\left(\varepsilon(j)=(-1)^{r-1}\right)
\end{aligned}
$$

for all $r \geqslant 0$, where we abbreviate $\tau_{\varepsilon}^{(r)}=\underbrace{\tau_{\varepsilon} \tau_{-\varepsilon} \tau_{\varepsilon} \cdots}_{r \text { factors }}$.

Substituting the expressions given by (12.17) into (8.12), we obtain explicit formulas for exchange relations in $\mathcal{A}^{\text {univ }}$ that belong to the bipartite belt. By comparing (12.17) with the expressions (10.3) and (10.4) for the denominator vectors, it is easy to see that if the exchange relation in question has $\left\{\beta, \beta^{\prime}\right\}=\left\{\tau_{+}(\alpha), \tau_{-}(\alpha)\right\}$, then the corresponding primitive coefficient $p\left(\left\{\beta, \beta^{\prime}\right\} ; \gamma_{1}\right)$ is equal to $p\left[\alpha^{\vee}\right]$, completing the proof. Note that there is also a nice formula for the other coefficient in the same relation:

$$
p\left(\left\{\beta, \beta^{\prime}\right\} ; \gamma_{2}\right)=\prod_{\beta^{\vee}} p\left[\beta^{\vee}\right]^{\left(\beta^{\vee} \| \alpha^{\vee}\right)},
$$

where $\beta^{\vee}$ runs over $\Phi_{\geqslant-1}^{\vee}$, and $\left(\beta^{\vee} \| \alpha^{\vee}\right)$ is the compatibility degree introduced in [FZ03b, $\S 3.1$ ]. We do not need this formula for the proof.

We continue the proof of Theorem 12.4. By Lemma 12.8, every generator $p\left[\alpha^{\vee}\right]$ of $\mathbb{P}^{\text {univ }}$ appears as a coefficient in an exchange relation on the bipartite belt. It follows that the coefficients in the exchange relations generate $\mathbb{P}^{\text {univ }}$ as a multiplicative group. In view of (12.3), we arrive at the following conclusion.

- If a cluster algebra $\mathcal{A}$ is obtained from $\mathcal{A}^{\text {univ }}$ by a coefficient specialization $\varphi$, then $\varphi$ is unique.

It remains to prove the existence of a coefficient specialization $\varphi: \mathcal{A}^{\text {univ }} \rightarrow \mathcal{A}$. To do this, we introduce the following terminology. 


\section{Cluster algebras IV}

Definition 12.9. A multiplicative coefficient identity (MCI) is a multiplicative relation among the coefficients $p\left(\left\{\beta, \beta^{\prime}\right\} ; \gamma\right)$ that holds in all cluster algebras of a given type, independently of the choice of coefficients.

The remaining (existence) part of Theorem 12.4 is an immediate consequence of Lemma 12.8 in conjunction with the following lemma.

Lemma 12.10. For every non-primitive coefficient $p\left(\left\{\beta, \beta^{\prime}\right\} ; \gamma\right)$, there is an MCI expressing it as a product of primitive coefficients (not necessarily distinct).

Proof. We start by deducing the desired statement from the following formally weaker assertion:

For every non-primitive coefficient $p\left(\left\{\beta, \beta^{\prime}\right\} ; \gamma\right)$, there is an MCI that

expresses it as a product of two or more other coefficients.

Indeed, let us assume that (12.19) holds, and fix an MCI as in (12.19) for each non-primitive coefficient $p\left(\left\{\beta, \beta^{\prime}\right\} ; \gamma\right)$. To prove that every non-primitive coefficient $p$ factors into a product of primitive coefficients, we write a chosen MCI for $p$ as $p=p_{1} \cdots p_{s}$, then substitute for each $p_{i}$ the product given by the corresponding MCI, and continue in the same way. To show that this process terminates, consider the following directed graph $\Gamma$ : the vertices of $\Gamma$ correspond to all of the coefficients $p\left(\left\{\beta, \beta^{\prime}\right\} ; \gamma\right)$ in the exchange relations, and the edges are of the form $p \rightarrow p^{\prime}$ whenever $p^{\prime}$ is one of the factors in the chosen MCI for $p$. Since the sinks of $\Gamma$ are precisely the primitive coefficients, it suffices to show that $\Gamma$ has no oriented cycles. Suppose on the contrary that $p_{1} \rightarrow p_{2} \rightarrow \cdots \rightarrow p_{s} \rightarrow p_{1}$ is an oriented cycle in $\Gamma$. Multiplying the chosen MCIs along this cycle and canceling $p_{1} \cdots p_{s}$, we obtain an MCI that expresses 1 as a product of several coefficients: $1=q_{1} \cdots q_{r}$. Being an MCI, this identity must be satisfied in any cluster algebra of the given type. Returning for a moment to the nomenclature of coefficients used in the preceding sections, we can write $q_{1}$ in the form $q_{1}=p_{j ; t}^{+}$for some $j \in[1, n]$ and $t \in \mathbb{T}_{n}$. Now let $\mathcal{A}$. be the algebra (of the same finite type) with principal coefficients at $t$. Its coefficient semifield is $\operatorname{Trop}\left(y_{1}, \ldots, y_{n}\right)$, with $q_{1}=y_{j}$, while the rest of the factors $q_{2}, \ldots, q_{r}$ are monomials in $y_{1}, \ldots, y_{n}$ (with non-negative exponents). Hence, the equality $1=q_{1} \cdots q_{r}$ does not hold in $\mathcal{A}_{\bullet}$ and so cannot be an MCI. This contradiction proves that Lemma 12.10 is indeed a consequence of (12.19).

The proof of (12.19) follows from the results in [FZ02]: applying the reduction procedure in $[F Z 02, \S 2]$, we reduce the statement to the rank 2 case, and then the desired MCI can be obtained as one of the identities [FZ02, (6.11)-(6.13)]. For the convenience of the reader, we provide a little more details here. Let us start with a relation of the form (12.13) with the coefficient $p_{1}$ nonprimitive. This means that the cluster monomial $x\left[\gamma_{2}\right]$ is non-trivial, hence contains some cluster variable $x\left[\beta^{\prime \prime}\right]$. Let $\mathbf{x}$ be a cluster containing $x[\beta]$ and such that $\mathbf{x}-\{x[\beta]\} \cup\left\{x\left[\beta^{\prime}\right]\right\}$ is another cluster. Let $\mathbf{z} \subset \mathbf{x}$ be the $(n-2)$-element subset $\mathbf{x}-\left\{x[\beta], x\left[\beta^{\prime \prime}\right]\right\}$. Following [FZ02, $\left.\S 2\right]$, we can consider the rank 2 cluster algebra setup with the initial cluster $\left\{x[\beta], x\left[\beta^{\prime \prime}\right]\right\}$ obtained by 'freezing' the cluster variables from $\mathbf{z}$, i.e. viewing them as 'constants' or 'new coefficients'. This algebra is of one of the finite types $A_{2}, B_{2}$ or $G_{2}$; the type $A_{1} \times A_{1}$ cannot occur because then all of the cluster variables occurring in $x\left[\gamma_{1}\right]$ or $x\left[\gamma_{2}\right]$ would belong to z. Its exchange graph is an $(h+2)$-cycle (cf. Theorem 8.8), that is, a 5-cycle (respectively, 6-cycle, 8-cycle) for the type $A_{2}$ (respectively, $B_{2}, G_{2}$ ). In determining the MCIs along this cycle, we can ignore the terms from $\mathbf{z}$, which reduces the proof of (12.19) to the rank 2 case, and brings us into the setup studied in [FZ02, §6].

Let us rewrite the results in [FZ02] in our current notation. Assume that $\Phi$ is of one of the types $A_{2}, B_{2}$, or $G_{2}$. Let $\Phi_{\geqslant-1}=\left\{\beta_{1}, \ldots, \beta_{h+2}\right\}$, where the roots are ordered cyclically around the origin; for instance, in type $A_{2}$ we use the same ordering as in (12.6). We use the convention that the subscripts are considered modulo $(h+2)$. Then the exchange relations and the corresponding MCIs can be described as follows. 


\section{S. Fomin And A. Zelevinsky}

TABLE 9. Index of conjectures.

\begin{tabular}{|c|c|c|}
\hline Conjecture & $\begin{array}{c}\text { Bipartite case; } \\
\text { seeds/variables on } \\
\text { the bipartite belt }\end{array}$ & $\begin{array}{c}\text { Finite type; } \\
\text { bipartite initial } \\
\text { seed }\end{array}$ \\
\hline $\begin{array}{c}\text { Conjecture } 4.3 \\
{[\mathrm{FZ} 04, \text { Conjecture } 4.14(1)]}\end{array}$ & & [FZ03a, Theorem 1.13] \\
\hline Conjectures $5.4-5.5$ & Corollary 10.10 & \\
\hline Conjecture 6.10 & Proposition 10.16 & \\
\hline Conjecture 6.11 & Corollary 10.10 & \\
\hline Conjecture 6.13 & Remark 10.14 & \\
\hline $\begin{array}{c}\text { Conjecture } 7.2 \\
{[\text { FZ04, Conjecture 4.16] }}\end{array}$ & & Theorem 11.2 \\
\hline Conjecture 7.4 & & [FZ03a, Theorems 1.9, 1.13] \\
\hline Conjecture 7.5 & Corollary 10.5 & \\
\hline $\begin{array}{c}\text { Conjecture } 7.6 \\
{[\text { FZ04, Conjecture 4.17] }}\end{array}$ & & Proposition 11.1 \\
\hline Conjecture 7.10 & & Corollary 11.4 \\
\hline Conjecture 7.12 & Proposition 10.16 & \\
\hline Conjecture 7.17 & Corollary 10.10 & \\
\hline
\end{tabular}

Type $A_{2}$. The exchange relations have the form

$$
x\left[\beta_{m-1}\right] x\left[\beta_{m+1}\right]=q_{m} x\left[\beta_{m}\right]+r_{m} .
$$

For every $m$, there is an MCI expressing the non-primitive coefficient $r_{m}$ as a product $r_{m}=$ $q_{m+2} q_{m+3}$.

Type $B_{2}$. In this case, in the sequence $\beta_{1}, \beta_{2}, \ldots$, the long and short roots alternate. The exchange relations have the form

$$
x\left[\beta_{m-1}\right] x\left[\beta_{m+1}\right]=q_{m} x\left[\beta_{m}\right]^{b_{m}}+r_{m},
$$

where $b_{m}=1$ (respectively, 2) if $\beta_{m-1}$ and $\beta_{m+1}$ are short (respectively, long). The corresponding MCIs look as follows: $r_{m}=q_{m+2} q_{m+3}^{b_{m}} q_{m+4}$.

Type $G_{2}$. Again, in the sequence $\beta_{1}, \beta_{2}, \ldots$, the long and short roots alternate. The exchange relations have the form

$$
x\left[\beta_{m-1}\right] x\left[\beta_{m+1}\right]=q_{m} x\left[\beta_{m}\right]^{b_{m}}+r_{m},
$$

where $b_{m}=1$ (respectively, 3 ) if $\beta_{m-1}$ and $\beta_{m+1}$ are short (respectively, long). The corresponding MCIs look as follows:

$$
r_{m}= \begin{cases}q_{m+2} q_{m+3} q_{m+4}^{2} q_{m+5} q_{m+6} & \text { if } \beta_{m-1} \text { and } \beta_{m+1} \text { are short; } \\ q_{m+2} q_{m+3}^{3} q_{m+4}^{2} q_{m+5}^{3} q_{m+6} & \text { if } \beta_{m-1} \text { and } \beta_{m+1} \text { are long. }\end{cases}
$$

This completes the proof of Lemma 12.10.

Theorem 12.4 is also proved. 


\section{Cluster algebras IV}

Remark 12.11. Once it is proved that the cluster monomials form a $\mathbb{Z} \mathbb{P}$-basis in any cluster algebra $\mathcal{A}$ of finite type (see [FZ04, Conjecture 4.28]), Theorem 12.4 will imply that any such $\mathcal{A}$ can be obtained by a 'base change' from the universal cluster algebra $\mathcal{A}^{\text {univ }}$ of the same type: that is, $\mathcal{A}$ is canonically isomorphic to $\mathcal{A}^{\text {univ }} \otimes_{\mathbb{Z} \mathbb{P} \text { univ }} \mathbb{Z} \mathbb{P}$.

\section{Index of conjectures}

Table 9 gives pointers to partial results which provide supporting evidence towards the conjectures proposed in this paper: either for bipartite initial seeds or under the additional assumption of finite type.

\section{ACKNOWLEDGEMENTS}

The authors thank the following institutions and colleagues whose hospitality and support stimulated the work on this research project: University of Bielefeld (Claus Michael Ringel); Frederick W. and Lois B. Gehring Fund and University of Michigan; Institut Fourier (Michel Brion); and Institut Mittag-Leffler (Anders Björner and Richard Stanley). We also thank Vladimir Fock, Alexander Goncharov, and Alek Vainshtein for stimulating discussions.

\section{REFERENCES}

BFZ96 A. Berenstein, S. Fomin and A. Zelevinsky, Parametrizations of canonical bases and totally positive matrices, Adv. Math. 122 (1996), 49-149.

BFZ05 A. Berenstein, S. Fomin and A. Zelevinsky, Cluster algebras III: Upper bounds and double Bruhat cells, Duke Math. J. 126 (2005), 1-52.

BLM89 S. Berman, Y. S. Lee and R. Moody, The spectrum of a Coxeter transformation, affine Coxeter transformations, and the defect map, J. Algebra 121 (1989), 339-357.

BGH02 E. Bolker, V. Guillemin and T. Holm, How is a graph like a manifold? Preprint (2002), math.CO/0206103.

CC04 P. Caldero and F. Chapoton, Cluster algebras as Hall algebras of quiver representations, Comment. Math. Helv. 81 (2006), 595-616.

CK05a P. Caldero and B. Keller, From triangulated categories to cluster algebras, Preprint (2005), math.RT/0506018.

CK05b P. Caldero and B. Keller, From triangulated categories to cluster algebras II, Preprint (2005), math.RT/0510251.

FG05 V. V. Fock and A. B. Goncharov, Dual Teichmüller and lamination spaces, Preprint (2005), math.DG/0510312.

FG03 V. V. Fock and A. B. Goncharov, Cluster ensembles, quantization and the dilogarithm, Preprint (2003), math.AG/0311245.

FR05 S. Fomin and N. Reading, Root systems and generalized associahedra, in Lecture notes for the IAS/Park City Graduate Summer School in Geometric Combinatorics, July 2004, Preprint (2005), math.CO/0505518.

FZ02 S. Fomin and A. Zelevinsky, Cluster algebras I: Foundations, J. Amer. Math. Soc. 15 (2002), 497-529.

FZ03a S. Fomin and A. Zelevinsky, Cluster algebras II: Finite type classification, Invent. Math. 154 (2003), $63-121$.

FZ03b S. Fomin and A. Zelevinsky, $Y$-systems and generalized associahedra, Ann. of Math. (2) 158 (2003), 977-1018.

FZ04 S. Fomin and A. Zelevinsky, Cluster algebras: notes for the CDM-03 conference, in CDM 2003: Current Developments in Mathematics (International Press, Boston, MA, 2004). 


\section{Cluster algebras IV}

FS95 E. Frenkel and A. Szenes, Thermodynamic Bethe ansatz and dilogarithm identities. I, Math. Res. Lett. 2 (1995), 677-693.

GSV03 M. Gekhtman, M. Shapiro and A. Vainshtein, Cluster algebras and Poisson geometry, Moscow Math. J. 3 (2003), 899-934.

GT96 F. Gliozzi and R. Tateo, Thermodynamic Bethe ansatz and three-fold triangulations, Internat. J. Modern Phys. A 11 (1996), 4051-4064.

GZ99 V. Guillemin and C. Zara, Equivariant de Rham theory and graphs, Asian J. Math. 3 (1999), 49-76.

How82 R. B. Howlett, Coxeter groups and M-matrices, Bull. London Math. Soc. 14 (1982), 137-141.

McM02 C. T. McMullen, Coxeter groups, Salem numbers and the Hilbert metric, Publ. Math. Inst. Hautes Études Sci. 95 (2002), 151-183.

SZ04 P. Sherman and A. Zelevinsky, Positivity and canonical bases in rank 2 cluster algebras of finite and affine types, Moscow Math. J. 4 (2004), 947-974.

Ste59 R. Steinberg, Finite reflection groups, Trans. Amer. Math. Soc. 91 (1959), 493-504.

Zam91 A. B. Zamolodchikov, On the thermodynamic Bethe ansatz equations for reflectionless ADE scattering theories, Phys. Lett. B 253 (1991), 391-394.

Sergey Fomin fomin@umich.edu

Department of Mathematics, University of Michigan, Ann Arbor, MI 48109, USA

Andrei Zelevinsky andrei@neu.edu

Department of Mathematics, Northeastern University, Boston, MA 02115, USA 